\title{
Combined Structural and Compositional Evolution of Planetary Rings Due to Micrometeoroid Impacts and Ballistic Transport
}

\author{
Paul R. Estrada \\ Carl Sagan Center, SETI Institute \\ 189 N. Bernardo Ave. \# 100, Mountain View, CA 94043 \\ Richard H. Durisen \\ Astronomy Department \\ University of Indiana, Bloomington, IN 47405 \\ Jeffrey N. Cuzzi \\ NASA Ames Research Center \\ Mail Stop 245-3, Moffett Field, CA 94035 \\ and \\ Demitri A. Morgan \\ USRA, NASA Ames Research Center \\ Mail Stop 245-3, Moffett Field, CA 94035 \\ Revised submission to Icarus: June, 2014 \\ 112 manuscript pages, including... \\ 17 Figures and 1 Table and 4 appendices
}


Proposed Running Title: "Modeling Structure and Composition"

Correspondence Address:

Paul R. Estrada

Carl Sagan Center, SETI Institute

189 N. Bernardo Ave. \# 100, Mountain View, CA 94043

E-mail: Paul.R.Estrada@nasa.gov

Phone: (650) 810-0214

FAX: (650) 604-6779 


\begin{abstract}
We introduce improved numerical techniques for simulating the structural and compositional evolution of planetary rings due to micrometeoroid bombardment and subsequent ballistic transport of impact ejecta. Our current, robust code is capable of modeling structural changes and pollution transport simultaneously over long times on both local and global scales. In this paper, we describe the methodology based on the original structural code of Durisen et al. (1989, Icarus 80, 136-166) and on the pollution transport code of Cuzzi and Estrada (1998, Icarus 132, 1-35). We provide demonstrative simulations to compare with, and extend upon previous work, as well as examples of how ballistic transport can maintain the observed structure in Saturn's rings using available Cassini occultation optical depth data. In particular, we explicitly verify the claim that the inner B (and presumably A) ring edge can be maintained over long periods of time due to an ejecta distribution that is heavily biased in the prograde direction through a balance between the sharpening effects of ballistic transport and the broadening effects of viscosity. We also see that a "ramp"-like feature forms over time just inside that edge. However, it does not remain linear for the duration of the runs presented here unless a less steep ejecta velocity distribution is adopted. We also model the $\mathrm{C}$ ring plateaus and find that their outer edges can be maintained at their observed sharpness for long periods due to ballistic transport. We hypothesize that the addition of a significant component of a retrograde-biased ejecta distribution may help explain the linearity of the ramp and is probably essential for maintaining the sharpness of $\mathrm{C}$ ring plateau inner edges. This component would arise for the subset of micrometeoroid impacts which are destructive rather than merely cratering. Such a distribution will be introduced in future work.
\end{abstract}

\title{
1 Introduction
}

The rings' huge surface area-to-mass ratio ensures that they are particularly susceptible to the effects of extrinsic meteoroid bombardment. Until recently, the mass of Saturn's rings was thought to be comparable to a Mimas mass (although see Charnoz et al., 2009). Based on this value, the area-to-mass ratio for Saturn's rings exceeds that of Mimas ${ }^{1}$ by $\gtrsim 10^{4}$. A consequence of this is that hypervelocity micrometeoroid impacts on the rings, depending on parameters, would likely erode them on timescales much shorter than the age of the solar system, if all the ejecta escaped (Durisen et al., 1989). More realistically, these impacts produce a large amount of particulate ejecta, the vast majority of which are ejected at speeds much less than the velocity needed to escape the rings. As a result, a copious exchange of ejecta between different ring regions occurs which leads to changes in ring structure and composition on both local and global scales (Durisen et al., 1989; Cuzzi and Estrada, 1998; Charnoz et al., 2009). This process, by which the rings evolve subsequent to meteoroid bombardment, is referred to as "ballistic transport" of impact ejecta (Ip, 1983; Durisen, 1984a,b; Lissauer, 1984).

In a series of papers, Durisen and colleagues (Durisen et al., 1989; 1992; 1996) developed

\footnotetext{
${ }^{1}$ For purpose of this illustration, all the mass is assumed to be in the $\mathrm{B}$ ring where the optical depth $\tau \geq 1$. The mass of Mimas is $\sim 4 \times 10^{22} \mathrm{~g}$.
} 
the first rigorous dynamical code to model ring structural evolution due to meteoroid bombardment and ballistic transport. They found that the influence of these processes on the rings could explain certain aspects of ring structure such as the fairly abrupt inner edges of the A and B rings, and the very similar "ramp" features which connect them to the Cassini division and $\mathrm{C}$ ring (see Fig. 1) respectively. These structures imply evolutionary times of $\gtrsim 100$ "gross erosion" times, where the gross erosion time $t_{\mathrm{G}}$ (see Table I for a list of parameters) is defined as the time a reference ring annulus of surface density $\sigma$ would disappear due to loss of ejected material if nothing returned (see below, and Sec. 2.2.1). In a complementary study, Cuzzi and Estrada (1998, hereafter CE98) developed a model for the evolution of composition while assuming constant structure. They calculated how the abundance of both intrinsic and extrinsic non-icy materials evolves over time as icy rings are bombarded by largely cometary material, and how these impurities are redistributed over the rings. CE98 found that they could simultaneously explain the albedo and color dichotomy between the $\mathrm{C}$ ring/Cassini division material versus the $\mathrm{A}$ ring/B ring material and the radial variation of color across the $\mathrm{C}$ ring/B ring transition in a time scale similar to that on which Durisen and colleagues explained structural evolution.

Two key quantities in ballistic transport are the impact yield $Y$ and the impacting micrometeoroid flux $\dot{\sigma}_{\text {im }}$. Both $Y$ and $\dot{\sigma}_{\text {im }}$ (see Table 1 and Sec. 2) are essential for providing more accurate age-dating of specific ring features, as well as the overall age of the rings themselves. The yield of a single impact, $Y$, which is defined as the ratio of ejecta mass to impactor mass, can be quite large depending on several factors (e.g., see Durisen, 1984b). The gross erosion time is expressed in terms of these quantities $t_{\mathrm{G}}=\sigma / Y \dot{\sigma}_{\text {im }}$; this definition serves as a handy reference, but in fact most ejecta are not lost (see Sec. 2.2.1). Moreover, this definition is appropriate for cratering impacts, but does not straightforwardly generalize to disruptive impacts.

The ejecta velocity distribution resulting from an impact depends on the hardness of the target and the angle of impact (Cuzzi and Durisen, 1990, hereafter CD90). If the target is powdery, yields can be in excess of $\sim 10^{5}-10^{6}$ for cratering (non-disruptive) impacts at normal incidence (e.g., Burns et al., 1984), while micrometeor-sized particles impacting into hard or granular surfaces can have yields as small as $\sim 10^{3}$ (Vedder, 1972). The ejecta velocities for the bulk material from cratering impacts tend to range from $\sim 1-10 \mathrm{~m} \mathrm{~s}^{-1}$, much less than the local orbital velocity within the rings (tens of kilometers per second). This means that, regardless of whether impacts are cratering or disruptive, the net mass gain or loss from the rings due to micrometeoroid bombardment is small compared to its redistribution from place to place. The net gain or loss needs to be considered only for very long exposure times (Charnoz et al., 2009) or regions where tiny charged ejecta can be swept into the planet (Northrop and Connerney, 1987).

Earlier estimates of the current micrometeoroid flux at Saturn vary significantly (e.g., Morfill et al., 1983; CE98; Landgraf et al., 2000), but all suggest that the rings could be impacted by close to their own mass (for the Mimas mass estimate) over the age of the Solar System. These estimates are largely based on the meteoroid mass fluxes measured by the Pioneer and Ulysses spacecraft between 5-10 AU (see, CE98, Fig. 17). Some hope for improving this estimate had recently surfaced from Galileo measurements of the flux at Jupiter. Sremčevíc et al. (2005) used an indirect technique to provide an estimate of 
the mass flux that may be off by at most a factor of $2-3$ (larger) compared to previous estimates and is comparable to that estimated by CE98 (see Sec. 2.2.1). However, some recent measurements of the mass flux at Saturn suggest that the flux may be considerably lower than previously thought (Kempf, private comm.), making $t_{\mathrm{G}}$ longer than what we generally assume in this work.

In a frame rotating at orbital velocity, the ejecta mass from an extrinsic micrometeoroid impact that is not disruptive is thrown predominantly in the prograde orbital direction. This result arises naturally from the tendency of projectiles to arrive with velocity vectors that are retrograde in the orbital sense, as azimuthally averaged over the rings (CD90). That is, the combination of the orbital motion of ring particles and the motion of Saturn through the micrometeoroid flux leads to a "headwind" of material that increases both the impacts on the leading faces of ring particles and the impacting velocities (see also Latter et al., 2012). In addition to mass, ejecta carry away with them angular momentum. Since most of the ejecta from a non-disruptive (cratering) impact are prograde, they tend to reimpact the rings at outer locations. Prograde ejecta are launched from their original radial location with more angular momentum than their parent ring particle, but less angular momentum than ring particles they may impact on their next ring crossing at some outer radius. The net result is to decrease angular momentum at the secondary impact location, leading to radial inward drift.

On the other hand, an impact that leads to complete disruption of a target ring particle into several fragments would likely produce the opposite effect because the fragment velocities are biased in the same direction as that of the impactor, resulting in a retrograde distribution (relative to Keplerian) with lower ejecta velocities than their prograde counterparts (Nakamura and Fujiwara, 1991; Paolicchi et al., 1989). In either case, the structure of the rings (i.e., optical depth $\tau$ and surface density $\sigma$ ) will have an effect on the rate of material drift. This is because the probability of ejecta absorption, which determines the actual ejecta mass that is reabsorbed by the rings instead of merely passing through them, depends on the local $\tau$, and its angular momentum depends linearly on $\sigma$ (CD90; CE98; Charnoz et al., 2009). In fact, there is a smaller, but systematic, tendency for infall to decrease the net angular momentum of rings (Ip, 1983; CD90; see below)

These processes have been used to estimate ring lifetimes in several ways. First, they will lead to angular momentum loss within the rings globally. For example, it has been estimated that the $\mathrm{C}$ ring would be lost to the planet in $\sim 10^{7}-10^{8}$ years (CD90) if no other mechanism were at work to sustain it. A similar age for the rings related to the loss of grains and molecules to Saturn's ionosphere generated by impacts was obtained by Northrop and Connerney $(1987)^{2}$. Second, carbon and silicate-rich meteoroid material also darkens and "pollutes" the almost entirely icy rings over time, an effect that provides a powerful means for estimating ring age. Doyle et al. (1989), and subsequently CE98, using a mass flux from Grün et al. (1985), noted that the relatively high albedo of the A and B rings was inconsistent with their having retained more than a small fraction of primitive, carbonaceous material they would have accreted over the age of the Solar System as compared to the expected fraction of order unity. CE98 further demonstrated using their pollution transport model

\footnotetext{
${ }^{2}$ It should be noted that resonant interactions with nearby ring-moons can also lead to significant angular momentum loss (e.g., Goldreich and Tremaine, 1982; Esposito, 1987; Poulet and Sicardy, 2001).
} 
that the relatively small amount of extrinsic darkening material needed to evolve the inner $\mathrm{B}$ ring and the $\mathrm{C}$ ring to their current broadband colors would also suggest a geologically young age for the $\mathrm{C}$ ring, similar to the time it would take to lose the ring based on angular momentum loss arguments. This would seem to pose a problem for the possibility that the rings are more massive than previously thought, unless the $\mathrm{C}$ ring we see today is some great-grandchild of an original ancestor (Charnoz et al., 2009, see Sec. 3.1).

In fact the $\mathrm{C}$ ring provides fertile ground for ballistic transport modeling because most of the structure in the $\mathrm{C}$ ring remains unexplained, and there are several candidates for ballistic transport signatures. For instance (see upper panel of Figure 1), Durisen et al. (1992) suggested that the sharp inner edge of the B ring was maintained by a balance between ballistic transport, which tends to sharpen low-to-high optical depth transitions, and the broadening effect of viscous transport. Likewise, these workers also found, using a prograde ejecta distribution (CD90), that the adjoining "ramp" structure on the low optical depth side of the $\mathrm{B}$ ring inner edge inevitably came about due to advective effects. A similar sharp edge connected to a ramp is present at the inner A ring/Cassini division boundary (Fig. 1, lower panel). In this paper, we examine these results in more detail and establish that these features are indeed products of ballistic transport.

The shapes of the C ring "plateau" peaks are also suggestive (see Colwell et al., 2009). Material tends to drift more quickly in low optical depth, featureless regions because the kinematic viscosity is weak there. This means that inwardly drifting material will begin to "pile up" which leads to a sharpening of an outer plateau edge. Likewise, retrograde ejected material would exhibit outward radial drift and would tend to lead to a pile up at the plateau's inner edge. A plateau might then exhibit a characteristic in which an outer (inner) edge has higher amplitude than the inner (outer) edge, with a ramp in optical depth between them, if prograde (retrograde) ejecta is the dominant component. The observed plateaus in Fig. 1 display these features to differing degrees. Interestingly, there appears to be some symmetry between inner/outer plateau edge structure about the Maxwell ringlet at around $87500 \mathrm{~km}$ ( $\sim 1.45 \mathrm{R}_{S}$, where $R_{S}=60330 \mathrm{~km}$ is the radius of Saturn). Whether this is a coincidence or not is unclear. It may be that some variable combination of particle size, which may even differ between plateaus and surrounding regions (Colwell et al., 2012; Marouf et al., 2012), and impact velocities may lead to a transition between primarily cratering impacts (prograde ejecta) versus primarily disruptive impacts (retrograde ejecta) at around $1.45 \mathrm{R}_{S}$ (Estrada and Durisen, 2011).

Despite these intriguing possibilities, the plateaus, as well as the undulating structure in the inner $\mathrm{C}$ ring and the semi-regular $\sim 80 \mathrm{~km}$ structure in the inner $\mathrm{B}$ ring, none of which are associated with known resonances (although see Hedman and Nicholson, [2013]), have yet to be modeled successfully in convincing detail. Although we do not study the issue explicitly in this paper, an instability associated with ballistic transport (hereafter, BTI, Durisen 1995; Latter et al., 2012; 2014a,b) can lead to the spontaneous creation of undulatory structure in an otherwise uniform ring if ring conditions fall within a specific range of optical depths and wavelengths. This process could play an important role in the $\mathrm{C}$ ring and inner B ring. Specifically, Durisen (1995) suggested that ballistic transport could cause the semi-regular structure seen in the inner B ring and possibly the longer wavelength $\mathrm{C}$ ring undulations without the inner edge being the driving mechanism. Latter et al. (2012; 
2014a,b) were able to confirm and extend on the analysis of Durisen (1995). The simulations we present in this paper do strongly suggest that the BTI is at work, but operating under a very nonuniform background structure, where it is not as well studied or understood.

Another important issue, which we briefly touch upon in this paper, is whether the rings of Saturn are much more massive than previously thought (Sec. 3.2). If so, high optical depth regions, i.e., the B ring where most of this extra mass must be hidden (e.g., Charnoz et al., 2009), should be able to resist pollution from extrinsic bombarding material even more easily than previously thought, and this should be manifested in current particle albedos. A more massive $\mathrm{B}$ ring does not necessarily indicate that all the ring elements are old, and indeed, the $\mathrm{C}$ ring probably cannot be long-lived. Charnoz et al. (2009) argue that the most likely time that the main rings formed would have been at the Late Heavy Bombardment (LHB, Gomes et al., 2005), setting up a mismatch between the age of the main rings and the age of the $\mathrm{C}$ ring.

A review of previous work on the subject of ballistic transport can be found in Charnoz et al. (2009). In this paper, we lay the groundwork for more detailed future modeling of micrometeoroid bombardment and ballistic transport in planetary rings through the introduction of an improved ballistic transport code that is capable of modeling both structure and composition in tandem. With this code, we are able to model the rings over a broad range of spatial scales and over both short and long timescales. These are important developments because the results of this work in combination with that of Latter et al. (2012; 2014a,b) strongly suggest that ballistic transport is operating within the rings, but it will be a more complicated endeavor to match all the observed features than previously envisioned, requiring additional physics that we have not yet incorporated in our model. Our improved code, though, is robust enough that new physics can readily be included, which will afford us the ability to explore this complexity in more detail.

In Section 2, we describe the development of our combined structural and compositional code. In Section 3, we present the results of demonstrative simulations which reproduce and extend upon previous work, as well as introduce new modeling of observed C ring structure. In Section 4 we present a discussion of our conclusions and, finally, in Section 5 we outline the direction of future work.

\section{Combined Structural and Compositional Model}

\subsection{Limitations of Previous Work}

Previous studies involving ballistic transport in Saturn's main rings were limited in two ways. From the structural standpoint, solving the ballistic transport equations (see Sec. 2.2.3) is computationally expensive, even with today's processing abilities. Ballistic transport essentially requires tracking the exchange of material from one location (radial bin) in the radial range of computation to every other radial location within its maximum "throw distance", which depends on the upper limit of the ejecta velocity distribution. Meanwhile, one must also sum up all the contributions to that radial bin from all other radial locations from which ejecta can reach it. Because this involves integration over multiple variables (e.g., angles, 
velocities, reabsorption probabilities) at every time over a potentially large histogram of radial bins, comprehensive parameter studies were not feasible, and global scale calculations intractable.

Another important handicap in previous studies was a lack of adequate data for some basic ring properties. For example, prior to Cassini, the ring optical depth $\tau(R)$ was known in most locations, but the ring surface density $\sigma(R)$ had only been measured in several isolated spots, which were generally associated with spiral density wave regions. It was then only possible to determine the ring opacity $\kappa(R)=\tau(R) / \sigma(R)$ in these discrete locations (e.g., Cuzzi et al., 1984; Lissauer and Cuzzi, 1985). CE98 extended these values to nondensity wave regions, assuming wave regions were not too different from non-wave regions, but could only estimate the spatial variability of $\kappa$ in optically thick regions because of a lack of occultation data (see Sec. 2.2.6). All of the simulations of Durisen et al. adopted the condition $\tau \propto \sigma$ and thus the opacity was assumed to have a constant value dependent on some reference value of $\sigma$ in the inner B ring (Sec. 3.1, also see Cuzzi et al., 1984).

A major limitation of the $\mathrm{C}$ ring/inner B ring compositional study of CE98 was their assumption that the underlying ring structure could be kept constant while the ring composition evolved, based on the finding by Durisen et al. (1992) that constant optical depth regions and inner sharp edges can remain more or less unchanged over long timescales. As a result, CE98 assumed that the optical depth and mass surface density were time-independent on the timescale of compositional evolution. This meant that the cumbersome $\tau$-dependent loss and gain integrals (see Sec. 2.2.5) that determine the redistribution of mass and angular momentum due to ballistic transport needed to be done only once which provided a considerable savings in computational time.

Cassini observations are providing a more detailed understanding of dynamical ring properties such as the opacity $\kappa$, surface density $\sigma$, and optical depth $\tau$ which are key quantities in modeling of ring structure. These observations, combined with numerical improvements will allow us to embark on much more detailed modeling and analyses than could have ever been done previously. On the other hand, Cassini has also shown us how local measurements of $\tau$ suggest a bimodal or multimodal "gap and clump" structure (i.e., self-gravity wakes) that we do not deal with explicitly here (e.g., Colwell et al., 2009) but hope to incorporate into future simulations.

\subsection{The Ballistic Transport Code}

The ballistic transport code (hereafter BT code) evolves the surface mass density of the rings $\sigma(R, t)$, and thus its structure, over time by solving the system of equations given below in Sec. 2.2.3. The code also tracks the changes in the fractional mass of extrinsic (bombarding) material, or "pollutant", which is assumed to be made up of some fraction of icy and non-icy constituents, and the rings' intrinsic material which is assumed to be composed mostly of water ice mixed with a very small fraction of absorbing material which is different from the micrometeoroid impactors. Thus the BT code also simultaneously evolves the bulk composition of the rings.

In ballistic transport, the two main effects that contribute to the rings' evolution are the 
direct net mass exchange of micrometeoroid impact ejecta between different ring regions (Ip, 1983), and the "indirect" compression or expansion arising from differential radial drift of material due to the direct net angular momentum exchange associated with the redistribution of impact ejecta (Lissauer, 1984; CD90). Secondary effects (e.g., mass loading, ring torque) that are related to the direct deposition of extrinsic material and its redistribution can also

play a role but are lesser in magnitude and more relevant over long timescales (Durisen et al., 1996; see Sec. 2.2.4).

The exchanges in mass and angular momentum $h$ per unit mass are determined at each radial location $R$ in a Lagrangian radial grid (i.e., one that moves with the material) by computing (1) the local gain rate in mass and angular momentum attributed to all of the extrinsic material and incoming ejecta from neighboring ring regions that are absorbed at $R$ and (2) the local loss rate in both quantities by accounting for the all of the ejecta created at $R$, which are ejected at different speeds and in different directions, that are absorbed at radial locations different from $R$. The net changes in $\sigma$ and $h$ at a given $R$ are then determined by differencing the local gains and losses. We call this combination of effects the "direct" contribution. Advection further modifies the local surface mass density through the compression or expansion of the Lagrangian cells based on differential radial drift velocities due to angular momentum readjustment, viscous angular momentum transport, and several other sources of radial drift. We call this the "indirect" contribution.

In this section we describe the motivation and mathematical formulation for the BT code. The most relevant parameters used are listed in Table 1. Much of what is presented below has been described elsewhere in detail (e.g., Durisen et al., 1989; 1992; 1996; CE98); however, given the mathematical and numerical focus of this paper, we believe it is justified to repeat some of these previous descriptions, while emphasizing differences in approach and new physics when appropriate. More detailed discussions of some of the various aspects of the BT code are included in the appendices; in particular, the numerical methodology of the code itself is described in Appendix D.

\subsubsection{The impact flux on the rings and definition of the gross erosion time}

Of the various input parameters for the modeling of ballistic transport in Saturn's rings, the micrometeoroid flux remains the most fundamental. For this work, we continue to use the value of the one-sided, incident flux at infinity $\dot{\sigma}_{\infty}=4.5 \times 10^{-17} \mathrm{~g} \mathrm{~cm}^{-2} \mathrm{~s}^{-1}$ used in CE98. This value was initially obtained from direct integration over the $1 \mathrm{AU}$ interplanetary flux models of Grün et al. (1985) by CD90, and was smaller than the value used by either Morfill et al. (1983) or Ip (1984). This value was used subsequently by Durisen and colleagues in later papers. CE98 reanalyzed and corrected the estimates from CD90 by including Pioneer 10/11 and Ulysses spacecraft data (see Fig. 17, CE98). CE98 found that the CD90 value for $\dot{\sigma}_{\infty}$ remained plausible within a factor of $2-3$, in spite of an inconsistency in the CD90 assumptions (see CE98 for a detailed discussion). The two-sided flux of micrometeorites impacting the ring plane and potentially absorbed at some radial location $R$ is given by $\dot{\sigma}_{\text {im }}$ :

$$
\dot{\sigma}_{\mathrm{im}}=2 \dot{\sigma}_{\infty} \mathscr{A}(\tau) F_{\mathrm{G}}\left(R / R_{0}\right)^{-0.8}
$$


where we have accounted for the gravitational focusing of the planet (see CD90) using an asymptotic relative velocity of $14 \mathrm{~km} \mathrm{~s}^{-1}$, which is numerically averaged into a factor $F_{\mathrm{G}}=3$ at a reference radius $R_{0}=1.8 \mathrm{R}_{S}$. The flux at other radii is obtained by the numerical fit $\left(R / R_{0}\right)^{-0.8}$ to the radial dependence of the calculated focusing (CD90; also see CE98). The optical depth dependent function

$$
\mathscr{A}(\tau)=\left[1-e^{-\left(\tau / \tau_{s}\right)^{p}}\right]^{1 / p}
$$

is a parameterization of a numerically determined impact probability that depends on $\tau$ and the various angular aberrations averaged over the orbit of the ring particles (CD90; Durisen et al., 1996). Here the fit parameters are $\tau_{s}=0.515$ and $p=1.0335$ (CE98).

The actual mass flux of ejected material $\dot{\sigma}_{\text {ej }}$ at reference radius $R_{0}$ is determined by integration over the full ejecta yield function $\mathscr{Y}$ derived by CD90 (see their equations 40$46)^{3}$ :

$$
\dot{\sigma}_{\mathrm{ej}}\left(R_{0}\right)=2 \dot{\sigma}_{\infty}\left(\frac{Y}{10^{4}}\right) \int_{x} \int_{\Omega} \mathscr{Y}\left(R_{0}, x, \Omega\right) d x d \Omega \approx Y \dot{\sigma}_{\mathrm{im}}\left(R_{0}\right),
$$

which depends on the ratio of the ejecta velocity to orbital velocity $x=v_{\mathrm{ej}} / v_{K}$, and solid angle $\Omega(\theta, \phi)$, where $\theta$ and $\phi$ are the zenith and azimuth angles of emergent ejecta as measured from the ring particle velocity vector in the frame of the planet and the ring normal. The ejecta distribution function $\mathscr{Y}$ contains all the information about impact and escape probabilities and about gravitational focusing and is normalized to a yield of $Y=10^{4}$ at $14 \mathrm{~km} \mathrm{~s}^{-1}$ impact velocity (Lange and Ahrens, 1987). We use the approximation on the RHS of Eq. (3) below for illustrative purposes. It typically differs by no more than tens of percent from the exact calculation obtained through direct integration of $\mathscr{Y}$, (CE98, see Appendix A) which we always utilize in the BT code.

The "gross erosion time" $t_{\mathrm{G}}$ is the fundamental time unit of ballistic transport and is defined as the time it would take for a ringlet of surface density $\sigma$ to be completely eroded away if no material returned.

$$
t_{\mathrm{G}}=\frac{\sigma}{\dot{\sigma}_{\mathrm{ej}}} \approx \frac{\sigma}{Y \dot{\sigma}_{\mathrm{im}}}
$$

For example, using the two-sided, gravitationally focused mass flux in Eq. (1) and an ejecta yield of $Y=10^{4}$, a reference ring annulus with $\sigma=100 \mathrm{~g} \mathrm{~cm}^{-2}$ would erode away in $t_{\mathrm{G}} \gtrsim 10^{6}$ years.

We normalize the time units of the BT code to the gross erosion time at a particular radius, allowing our simulations to be independent of specific values of the micrometeoroid flux and yield. For specificity and ease of comparison, the evolutionary times for this paper are in the same units as those used in past papers (e.g., Durisen et al., 1992; CE98)

\footnotetext{
${ }^{3}$ Note that in CD90, the fitted solution to $\mathscr{Y}$ is expressed in terms of the cone and clock angles of the emergent ejecta.
} 


$$
t_{\mathrm{G}} \approx 1.3 \times 10^{5}\left(\frac{10^{5}}{Y}\right)\left(\frac{4.5 \times 10^{-17} \mathrm{~g} \mathrm{~cm}^{-2} \mathrm{~s}^{-1}}{\dot{\sigma}_{\mathrm{im}}}\right)\left(\frac{\sigma(\tau=1)}{96 \mathrm{~g} \mathrm{~cm}^{-2}}\right) \text { years }
$$

which has been defined for $\tau=1$ at a radius of $R=1.52 \mathrm{R}_{S}$ which corresponds to the $\mathrm{B}$ ring inner edge. Defining $t_{\mathrm{G}}$ at this radius was sensible for the edge simulations of Durisen and colleagues, but one must keep in mind that the gross erosion times for the lower optical regions of the $\mathrm{C}$ ring (which range from $\tau \sim 0.05-0.5$ ) will be shorter than the time given in Eq. (5). In any case, the absolute ages derived from our simulations remain uncertain because of uncertainties in $\dot{\sigma}_{\mathrm{im}}, Y$, and the efficiency with which extrinsic bombarding material retains its absorptive properties.

\subsubsection{Code assumptions}

Basics. The rings are modeled as a Keplerian thin disk that is axially symmetric, with the central planet taken to be a point mass, and ring self-gravity is ignored. A thin disk is a reasonable assumption because the velocity dispersion $c$ of ring particles is typically a few millimeters per second (Schmidt et al., 2009; Colwell et al., 2009) somewhat akin to the escape velocity from several-meter-sized particles, which are the sizes observed to be the upper bound in the particle size distribution (Cuzzi et al., 2009). On the other hand, the orbital speeds of ring particles $v_{K}$ are on the order of tens of kilometers per second. The random motions due to $c$ lead to a vertical thickness $H / R \sim c / v_{K} \ll 1$, or an $H$ of a few times our largest particle size (Cuzzi et al., 1979).

To consider the disk to be thin with respect to ballistic transport, the radial and vertical excursions of impact ejecta should be much greater than $H$. Ejecta velocities $v_{\text {ej }}$ from nondisruptive impacts can vary from $\sim 1-100 \mathrm{~m} \mathrm{~s}^{-1}$ depending on the hardness of the target (e.g., Lange and Ahrens, 1982; Hartmann, 1985). The inward or outward radial excursion of an ejecta particle, which we refer to as its 'throw distance', is given by $|\delta R| \approx 4 x R$ (Morfill et al., 1983; Durisen et al., 1992). Comparing the radial throw distance to the ring vertical thickness, $|\delta R| / H \sim 4 x R / R\left(c / v_{K}\right) \sim 4 v_{\mathrm{ej}} / c \gg 1$. By the same token, the average vertical excursion is $|\delta z| \sim v_{\mathrm{ej}} P / 2 \sim \pi R\left(v_{\mathrm{ej}} / v_{K}\right)$, where $P$ is the orbital period, so that again we find $|\delta z| / H \sim \pi v_{\mathrm{ej}} / c \gg 1$

Our assumption of axial symmetry stems from a comparison of timescales. The typical orbital period of a ring particle is on the order of ten hours, whereas the relevant timescales of ballistic transport are on the order of $t_{\mathrm{G}} \sim 10^{4}-10^{6}$ years. Given this disparity, it is reasonable to expect that transient deviations from axial symmetry in underlying ring structure due to impacts average out over the timescales involved in our simulations. We use a variable timestep in our BT code (see Appendix D) which could be as short as a few years if the ring ambient conditions in the simulation call for it. This still equates to $\gtrsim 10^{3}$ orbits which should not invalidate our assumption. We leave consideration of the much more complicated problem of 2-D ballistic transport modeling (e.g., allowing explicitly for non-axisymmetric wakes [Colwell et al., 2009]) for future study.

Ring self-gravity is considered to be unimportant with respect to impact ejecta because the escape velocity from the ring particle size that dominates the Saturn ring mass, a few meters, is much smaller than the typical ejection speeds we use here. The presence of larger 
moonlets whose masses are large enough to produce escape velocities on the same order as that of the typical ejectum are also not considered, because these objects are sparse and thus would only effect a very small fraction of ejecta at any given time. Saturn's non-sphericity can lead to nodal precession and apsidal motion on timescales of tens of days in the $\mathrm{C}$ ring, with timescales increasing with radial location (Lissauer and Cuzzi, 1982). Given the relatively short orbital times of ring particles, ejecta are expected to complete at most a few orbits before being reabsorbed (Durisen et al., 1989; CD90). Thus, Saturn's oblateness should make little difference for ejecta trajectories and is neglected.

Ejecta distribution. We assume that all ejecta travel on Keplerian elliptical orbits between their point of ejection and their point of eventual reabsorption, and reabsorbed ejecta do not produce secondary ejecta distributions. The former assumption is valid because $x \ll 1$, so the ejecta travel on slightly elliptical orbits and cannot escape the system but rather reimpact the ring at distances up to the maximum throw distance $\sim 4 x_{\max } R$ and in timescales much less than the orbit precession timescale. The latter assertion seems sensible in that the secondary impacts of ejecta grains with the grainy regoliths of ring particles would be at less than $\sim 100 \mathrm{~m} \mathrm{~s}^{-1}$ velocities instead of tens of kilometers per second and likely to be highly inelastic (e.g., Hartmann, 1985). The secondary ejecta velocities are thus expected to be $\ll v_{\mathrm{ej}}$, with reimpacts occurring locally, within the maximum radial bin resolution we use in the BT code ( $\gtrsim$ a few kilometers).

Ring and meteoroid composition. The intrinsic composition of the rings is assumed to be primarily water-ice with a small fraction of spectrally red, absorbing material that is "intramixed" within the ice grains. CE98 found that Titan tholins provided a good fit to the observations, however other candidates for the reddish "UV-absorber" have been proposed (e.g., Cuzzi et al., 2009; Clark et al., 2012). The extrinsic, bombarding material is assumed to contain a fraction $f_{\text {ext }} \sim 0.5$ of a spectrally neutral, non-icy darkening agent, with the remainder being water ice. The BT code tracks the mass fraction $f_{\mathrm{e}}(R, t)$ of non-icy extrinsic material in the rings and assumes that the remainder $\left(1-f_{\mathrm{e}}\right)$ is the admixture of primordial and extrinsic icy component. Extrinsic absorbing material is assumed to be retained locally in the ring with an efficiency $\eta$ (see, e.g., Doyle et al., 1989; CE98), and uniformly mixed throughout the local mass density $\sigma(R, t)$. Thus, all the components of composition are assumed to be volumetrically mixed within the ring particles at all times.

This assumption is more than likely adequate over long timescales ( $>$ a few $t_{\mathrm{G}}$ ). However, a more recent model of regolith growth in ring particles due to bombardment by a broader size range of projectiles (up to meter size) suggests that, for ring particles that initially begin as pure ice, their regoliths remain more or less constant in the fractional amount of pollutant for timescales $\lesssim 1 \mathrm{t}_{G}$ as they grow in depth (Elliott and Esposito, 2011). Once the regolith has fully developed and essentially fills the particle, fractional mass increases linearly with time which is consistent with this work. The work of Elliott and Esposito (2011) becomes particularly relevant when considering the study of shorter timescale, transient features. For example, a recent local event such as the breakup of a small icy moon may lead to the resetting, locally, of the ballistic transport "clock", an idea that has already been suggested to explain variations in brightness in the rings over length scales of $\sim 1000-3000 \mathrm{~km}$ that do not seem consistent with ballistic transport acting over long timescales (Cuzzi et al., 1984; Esposito et al., 2005). In future work, we plan to incorporate these effects into our BT code. 
In general though, the gardening process modeled by Elliott and Esposito (2011) validates our assumption that a ring particle's composition is well mixed and not characterized by their surface veneers.

Conservation of energy. Finally, the ballistic transport equations (Sec. 2.2.3) account for conservation of mass and angular momentum, but not of energy (Durisen et al., 1989). The reason is that micrometeoroid impacts play little if any role in maintaining the velocity dispersion in the rings against damping due to collisions, on energetics grounds. To see this, we consider the impact of a projectile of mass $m_{\mathrm{im}}$ with a ring particle of mass $m$. Through momentum exchange arguments, the random velocity imparted to a ring particle $m$ through the impact with a particle $m_{\mathrm{im}}$ is $\Delta v \sim Y \mu_{\mathrm{m}} v_{\mathrm{ej}}$, where $\mu_{\mathrm{m}}$ is the mass ratio $m_{\mathrm{im}} /\left(m+m_{\mathrm{im}}\right)$. The ring particle $m$ is impacted on a timescale

$$
t_{\mathrm{im}} \approx \frac{m_{\mathrm{im}} \sigma}{m \dot{\sigma}_{\mathrm{im}}} \approx \mu_{\mathrm{m}} \frac{\sigma}{\dot{\sigma}_{\mathrm{im}}}=\left(\mu_{\mathrm{m}} Y\right) \mathrm{t}_{G}
$$

Then, the rate at which specific energy is introduced into the system as a result of these impacts is $\Delta v^{2} / t_{\mathrm{im}}$, which can be compared with the rate at which specific energy is dissipated due to inelastic collisions (Cuzzi et al., 1979)

$$
\frac{\Delta v^{2}}{t_{\mathrm{im}}}=\mu_{\mathrm{m}} Y \frac{v_{\mathrm{ej}}^{2}}{t_{\mathrm{G}}} \gtrsim \frac{c^{2} \tau \Omega\left(1-\epsilon^{2}\right)}{3 \pi} \quad \rightarrow \quad \mu_{\mathrm{m}} \gtrsim 1.25 \times 10^{-12} \frac{c^{2} \sigma \tau\left(1-\epsilon^{2}\right)}{x^{2}},
$$

where $\epsilon$ is the coefficient of restitution ${ }^{4}$. Using our canonical choice for upper size cutoff $r_{2}=3 \mathrm{~m}$ as the size for $m$ and $c \sim 0.2 \mathrm{~cm} \mathrm{~s}^{-1}$ and considering typical parameters in our simulations for a $\mathrm{C}$ ring plateau, $\tau=0.4, \sigma=40 \mathrm{~g} \mathrm{~cm}^{-2}$ and $x=10^{-3}$, one finds that $r_{\mathrm{im}} \gtrsim 3\left(1-\epsilon^{2}\right)^{1 / 3} \mathrm{~cm}$. The mass peak in the interplanetary micrometeoroid flux, which is presumed to be cometary in origin, is around a size of $100 \mu \mathrm{m}$ (e.g., Grün et al., 1985; CE98), while Edgeworth-Kuiper belt dust particles have a mass peak between sizes of $1-10$ $\mu \mathrm{m}$ (Poppe et al., 2010; Poppe and Horányi, 2012). Although ring particle collisions are moderately elastic, coefficients of restitution very close to unity would be needed for impacts by micrometeorites to be able to maintain the observed velocity dispersion in the rings. Thus we assume that the velocity dispersion in the rings is maintained by a balance of collisional energy loss and viscous gain, which we do not model, and, as a consequence, we do not consider conservation of energy in our system of equations (Sec. 2.2.3).

\subsubsection{Working equations}

Given our set of assumptions from Sec. 2.2.2, the ring system is described at any time $t$ by a surface mass density $\sigma(R, t)$ and normal optical depth $\tau(R, t)$, where the two quantities are related through the ring opacity $\kappa(R, t)=\tau / \sigma$. The opacity may explicitly or implicitly incorporate information or assumptions about the ring particle density $\rho$ and size distribution.

\footnotetext{
${ }^{4}$ Alternatively, one can compare the specific energy introduced into the system to the viscous dissipation energy rate (e.g., Araki and Tremaine, 1986; Latter and Ogilvie, 2008).
} 
The evolution of the ring system under ballistic transport is determined through solving the following set of equations. The mass continuity equation

$$
\frac{\partial \sigma}{\partial t}+\frac{1}{R} \frac{\partial}{\partial R}\left(\sigma R v_{\mathrm{R}}\right)=\Gamma_{\mathrm{m}}-\Lambda_{\mathrm{m}}+\dot{\sigma}_{\mathrm{im}},
$$

or, alternatively, the areal angular momentum density continuity equation

$$
\frac{\partial}{\partial t}\left(\sigma h_{c}\right)+\frac{1}{R} \frac{\partial}{\partial R}\left[R\left(\sigma h_{c} v_{\mathrm{R}}\right)-\sigma \nu R^{3} \frac{d}{d R}\left(\frac{h_{c}}{R^{2}}\right)\right]=\Gamma_{\mathrm{h}}-\Lambda_{\mathrm{h}}+\left(\frac{\partial j}{\partial t}\right)_{\mathrm{im}},
$$

where $h_{c}=R v_{K}=(G M R)^{1 / 2}$ is the specific angular momentum for circular motion, $G$ is the gravitational constant and $M$ is the mass of the planet. Equation (8) follows the local changes in $\sigma$ and $R$ due to the net loss or gain of ejecta to and from other ring regions. The RHS of Eq. (8) accounts for the direct mass gains $\Gamma_{\mathrm{m}}$ and losses $\Lambda_{\mathrm{m}}$ by integrating the ejecta distribution over the rings taking into account cylindrical effects, while the RHS of Eq. (9) are the angular momentum loss and gain integrals (see Sec. 2.2.5). The divergence of the radial mass flux in the ring plane caused by the slow radial drift of ring material at velocity $v_{\mathrm{R}}$ brought on by angular momentum transport (indirect contribution) is accounted for in the second term on the LHS of (8).

Changes in the composition of the rings in terms of the mass fraction of non-icy, extrinsic material $f_{\mathrm{e}}(R, t)$ are followed using the equations for the evolution of the surface mass density of the extrinsic and intrinsic components

$$
\begin{gathered}
\frac{\partial \sigma_{\mathrm{e}}}{\partial t}+\frac{1}{R} \frac{\partial}{\partial R}\left(\sigma_{\mathrm{e}} R v_{\mathrm{R}}\right)=\eta f_{\mathrm{ext}} \dot{\sigma}_{\mathrm{im}}+\Gamma_{\mathrm{m}, \mathrm{e}}-f_{\mathrm{e}}(r, t) \Lambda_{\mathrm{m}}=\eta f_{\mathrm{ext}} \dot{\sigma}_{\mathrm{im}}+\Delta \dot{\sigma}_{\mathrm{e}} \\
\frac{\partial \sigma_{\mathrm{i}}}{\partial t}+\frac{1}{R} \frac{\partial}{\partial R}\left(\sigma_{\mathrm{i}} R v_{\mathrm{R}}\right)=\left(1-\eta f_{\mathrm{ext}}\right) \dot{\sigma}_{\mathrm{im}}+\Gamma_{\mathrm{m}, \mathrm{i}}-\left[1-f_{\mathrm{e}}(r, t)\right] \Lambda_{\mathrm{m}}=\left(1-\eta f_{\mathrm{ext}}\right) \dot{\sigma}_{\mathrm{im}}+\Delta \dot{\sigma}_{\mathrm{i}}
\end{gathered}
$$

where $\sigma=\sigma_{\mathrm{e}}+\sigma_{\mathrm{i}}$. Unless otherwise noted, for the simulations in this paper we choose $f_{\text {ext }}=0.5$ and $\eta=0.12$ which is consistent with CE98. The radial drift velocity $v_{\mathrm{R}}$ is discussed in the next section. The gain integrals $\Gamma_{\mathrm{m}, \mathrm{e}}$ and $\Gamma_{\mathrm{m}, \mathrm{i}}$ are described in Sec. 2.2.5.

\subsubsection{The Advective Term}

The total radial drift velocity $v_{\mathrm{R}}=v_{\mathrm{R}}^{\text {ball }}+v_{\mathrm{R}}^{\text {visc }}+v_{\mathrm{R}}^{\text {masl }}+v_{\mathrm{R}}^{\text {torq }}$ that appears in the indirect term (e.g., second term on the LHS of Eq. [8]) has multiple contributions, the most important being that due to the ballistic transport mechanism itself:

$$
v_{\mathrm{R}}^{\text {ball }}=\left(\sigma \frac{d h_{\mathrm{c}}}{d R}\right)^{-1}\left[\Gamma_{\mathrm{h}}-\Lambda_{\mathrm{h}}-h_{\mathrm{c}}\left(\Gamma_{\mathrm{m}}-\Lambda_{\mathrm{m}}\right)\right]
$$

Equation (12) can readily be derived from combining Eqns. (8) and (9) for the inviscid case where mass loading and net torques are ignored. A second component of radial drift 
$v_{\mathrm{R}}^{\text {visc }}$ is due to the viscous angular momentum transport. For a thin viscous Keplerian disk (Hartmann, 2009),

$$
v_{\mathrm{R}}^{\mathrm{visc}}=-\frac{3}{\sigma h_{\mathrm{c}}} \frac{\partial}{\partial R}\left(h_{\mathrm{c}} \sigma \nu\right),
$$

where $\nu(R, t)$ is the kinematic viscosity, which is discussed in detail in Sec. 2.2.7.

Secondary contributions to the radial drift of material that are due to the collective effects of meteoroids (mass loading and asymmetric absorption of ejecta, Durisen et al., 1996) are also included in the calculation of the total $v_{\mathrm{R}}$ in our BT code. Meteoroid bombardment leads to the direct deposition of mass, which is accounted for through $\dot{\sigma}_{\mathrm{im}}$. However a more important effect of this bombardment is the change it imparts to the local specific angular momentum $h$. An annulus subject to these effects will drift radially, adjusting its $h$ to match that of the equilibrium $h=h_{\mathrm{c}}$ at a different radius, with drift velocities due to these collective effects $v_{\mathrm{R}}^{\text {coll }}$ given by (Durisen et al., 1996; cf. Eq. [12])

$$
v_{\mathrm{R}}^{\text {coll }}=v_{\mathrm{R}}^{\text {torq }}+v_{\mathrm{R}}^{\text {masl }}=\left(\sigma \frac{d h_{\mathrm{c}}}{d R}\right)^{-1}\left[\left(\frac{\partial j}{\partial t}\right)_{\mathrm{im}}-h_{\mathrm{c}} \dot{\sigma}_{\mathrm{im}}\right],
$$

where the first term in the RHS of (14) is the meteoroid angular momentum deposition rate per unit area and time, and the second term on the RHS is the mass deposition rate. Secondary contributions are discussed in more detail in Appendix B.

\subsubsection{Computation of losses and gains}

The essence of the ballistic transport process lies in the changes of the local surface mass density due to the differences between losses and gains of ejecta material at some $R$ and the resultant drifts. The differences are of order $\sim x^{2}$ away from sharp edges, and $\sim x$ at sharp edges, and there are order $\sim x^{2}$ differences between the radial velocities $v_{\mathrm{R}}$ across neighboring ring regions through the divergence term. Quite literally, ballistic transport depends on differences of small differences between losses and gains (Durisen et al., 1989). The losses $\Lambda$ and gains $\Gamma$ in mass and angular momentum per unit area and time must be computed every time step, and at each radial location within the ring. The losses in mass and angular momentum must account for the total rate at which material and its associated angular momentum is being carried from ring radius $R$, in all possible directions and at all possible speeds, to other locations $R^{\prime}$ where the ejecta are reabsorbed:

$$
\begin{gathered}
\Lambda_{\mathrm{m}}=\mathscr{R}(R, \tau) \int_{0}^{\infty} d x \int_{0}^{2 \pi} d \phi \int_{-1}^{1} \mathscr{P}\left(R, R^{\prime}\right) F(x, \theta, \phi) d \cos \theta \\
\Lambda_{\mathrm{h}}=h_{\mathrm{c}} \mathscr{R}(R, \tau) \int_{0}^{\infty} d x \int_{0}^{2 \pi} d \phi \int_{-1}^{1}(1+x \cos \theta) \mathscr{P}\left(R, R^{\prime}\right) F(x, \theta, \phi) d \cos \theta .
\end{gathered}
$$

Here, $\mathscr{R}$ gives the total rate of mass ejection at radius $R$ per unit area, and $\theta$ and $\phi$ have been defined previously in Sec. 2.2.2. The function $F(x, \theta, \phi)$ specifies the distribution of ejecta 
that escape $R$, as a function of velocity and solid angle defined by $\theta$ and $\phi$. By definition, the integral $\iiint F(x, \theta, \phi) d x d \phi d \cos \theta \equiv 1$. The probability function $\mathscr{P}\left(R, R^{\prime}\right)$ is given by

$$
\mathscr{P}\left(R, R^{\prime}\right)=\frac{1-e^{-\tau_{z}^{\prime}}}{1-e^{-\left(\tau_{\mathbf{z}}+\tau_{z}^{\prime}\right)}}
$$

where $\tau_{\mathrm{z}}$ and $\tau_{\mathrm{z}}^{\prime}$ are the slant optical depths for the angles of incidence in the rotating frame measured from the ring plane normal at $R$ and $R^{\prime}$, respectively (see Eqns. [29] and [30], Durisen et al., 1989). Equation (17) gives the total probability that the ejected particle will be absorbed at $R^{\prime}$ rather than at $R$.

The corresponding gains in mass and angular momentum per unit area and time at the radial location $R$ are calculated with the integrals $\Gamma_{\mathrm{m}}$ and $\Gamma_{\mathrm{h}}$, which account for all the ejecta from other ring regions $R^{\prime}$ that reach $R$ and are absorbed there. Because ballistic transport is sensitive to the small net differences between the losses and gains, Durisen et al. (1989) argued that the gain integrals should be as closely analogous to Eqns. (15) and (16) as possible for purposes of numerical accuracy. This was accomplished by relating the

radius of ejection $R$ to all possible radii of absorption $R^{\prime}$ using a mathematical description of the Keplerian elliptical orbit followed by an ejectum $R^{\prime}=R A(x, \theta, \phi)$, where the function $A$ is given by Eq. (33) of Durisen et al. (1996). Summing up all of the contributions to the ring annulus $2 \pi R d R$ from all annuli $2 \pi R^{\prime} d R^{\prime}$ then leads to the following expressions for the mass and angular momentum gain integrals

$$
\begin{gathered}
\Gamma_{\mathrm{m}}=\int_{0}^{\infty} d x \int_{0}^{2 \pi} d \phi \int_{-1}^{1} \mathscr{R}\left(R^{\prime}, \tau^{\prime}\right) A^{2} \mathscr{P}\left(R^{\prime}, R\right) F^{\prime}(x, \theta, \phi) d \cos \theta, \\
\Gamma_{\mathrm{h}}=\int_{0}^{\infty} d x \int_{0}^{2 \pi} d \phi \int_{-1}^{1} h_{\mathrm{c}}^{\prime}(1+x \cos \theta) \mathscr{R}\left(R^{\prime}, \tau^{\prime}\right) A^{2} \mathscr{P}\left(R^{\prime}, R\right) F^{\prime}(x, \theta, \phi) d \cos \theta,
\end{gathered}
$$

where quantities within the integrals are evaluated at $R^{\prime}$, which is itself evaluated in terms of $x, \theta$ and $\phi$. The gain integral $\Gamma_{\mathrm{m}}$ can be broken into the fractional mass gain integrals $\Gamma_{\mathrm{m}, \mathrm{e}}$ and $\Gamma_{\mathrm{m}, \mathrm{i}}$, which incorporate the mass fractions of extrinsic and intrinsic materials, but are nonetheless similar to Eq. (18). For example,

$$
\Gamma_{\mathrm{m}, \mathrm{e}}=\int_{0}^{\infty} d x \int_{0}^{2 \pi} d \phi \int_{-1}^{1} f_{\mathrm{e}}\left(R^{\prime}, t\right) \mathscr{R}\left(R^{\prime}, \tau^{\prime}\right) A^{2} \mathscr{P}\left(R^{\prime}, R\right) F^{\prime}(x, \theta, \phi) d \cos \theta,
$$

and the analogous expression for $\Gamma_{\mathrm{m}, \mathrm{i}}$ follows from replacing $f_{\mathrm{e}}\left(R^{\prime}, t\right)$ with $1-f_{\mathrm{e}}\left(R^{\prime}, t\right)$. In Appendix D we describe our technique for solving these integrals numerically.

\subsubsection{Ring opacity models and particle size}

Knowing both the surface mass density and the optical depth allows one to calculate the ring opacity $\kappa$. In the work of CE98, who investigated whether pollution transport alone could explain the detailed shape of the ring color profiles across the rings, only two such locations were known in the inner $\mathrm{B}$ ring and the $\mathrm{C}$ ring: the Janus 2:1 density wave and the Maxwell 
ringlet, respectively. In order to achieve their goal, CE98 required an opacity profile with higher radial resolution. They assumed the particle size distribution is best described by a power law in radius $r_{p}(R, t) \propto r^{-3}$ in most regions with upper and lower cutoffs $r_{1}, r_{2}$ of $3 \mathrm{~cm}$ and $3 \mathrm{~m}$, respectively (Zebker et al., 1985; Cuzzi et al., 2009), and we continue to use this as our canonical size distribution. Given this distribution, $\kappa$ depends primarily on the largest particle size ${ }^{5}$. Under this assumption, CE98 (see their Fig. 5) utilized the variance technique of Showalter and Nicholson (1990), which determines the largest "particle" size with high radial resolution, to determine from the $\delta$-Sco Voyager stellar occultation the largest effective radius particle across a range of ring radii where no spiral density waves existed. The scatter in their derived $\kappa$ values, coupled with values inferred by others, led CE98 to adopt very simple $\kappa(R)$ profiles for the $\mathrm{C}$ ring and inner $\mathrm{B}$ ring simulations:

$$
\kappa(R)= \begin{cases}\kappa_{1} & R \leq R_{1} ; \\ \kappa_{1}-\frac{\kappa_{1}-\kappa_{2}}{R_{2}-R_{1}}\left(R-R_{1}\right) & R_{1}<R<R_{2} ; \\ \kappa_{2} & R \geq R_{2} .\end{cases}
$$

where (in units of $\mathrm{cm}^{2} \mathrm{~g}^{-1}$ ) $\kappa_{1}=0.05, \kappa_{2}=0.009, R_{1}=1.5 \mathrm{R}_{S}$ (roughly the base of the $\mathrm{C}$ ring ramp, see Fig. 1), and $R_{2}$ was modestly varied to different locations in the very inner $\mathrm{B}$ ring. The estimated $\kappa=\kappa_{2}$ for the $\mathrm{B}$ ring opacity was actually an average over a range of possible values, due to a lack of occultation data in optically thick regions (see Fig. 11 of Showalter and Nicholson 1990). Recall that the treatment of CE98 (and below) differs from that of Durisen and colleagues who effectively used a constant opacity in their models (e.g., see Durisen et al., 1992; Sec. 2.1 and 3.1).

One observation that CE98 gleaned from point-by-point inspection of their derived opacity values in the $\mathrm{C}$ ring was that these values indicated a preferential association of low $\kappa$ (larger particles) with the relatively higher optical depth plateaus, while higher $\kappa$ were associated with regions away from plateaus where $\tau$ was lower. This was somewhat supported at the time by comparison of Voyager visual and radio wavelength optical depths. If true, this effectively would say that there is more mass in the plateaus than the simple model in Eq. (21) would predict. This suggests an alternative model for the ring opacity that we utilize for this work, namely that the opacity depends on $\tau$ (or alternatively, $\sigma$ ). For models that explore the $\mathrm{C}$ ring and inner $\mathrm{B}$ ring, we can define the initial values of the opacity $\kappa(R, 0)=a+b / \tau(R, 0)$ and at all later times through

$$
\kappa(R, t)=\frac{a}{2}+\frac{a}{2}\left[1+\frac{4 b}{a^{2} \sigma(R, t)}\right]^{1 / 2},
$$

where the fit parameters $a=\kappa_{1}+(\Delta \kappa / \Delta \tau) \tau_{2}, b=-(\Delta \kappa / \Delta \tau) \tau_{1} \tau_{2}, \Delta \kappa=\kappa_{2}-\kappa_{1}$ and $\Delta \tau=\tau_{2}-\tau_{1}$. The values of $\kappa_{1,2}$ and $\tau_{1,2}$ can readily be chosen to be consistent with the profile given in Eq. (21). Thus, for $\tau_{1}=0.05$ and $\tau_{2}=1, a \simeq 0.0068$ and $b \simeq 0.0022$ (both $\left.\mathrm{cm}^{2} \mathrm{~g}^{-1}\right)$, respectively. The above formula is heuristic, but it allows us to associate a certain optical depth with a specific surface mass density. This in turn allows us to model plateaus

\footnotetext{
${ }^{5}$ For power law slopes $>3$, this assumption is not true because the lower bound of the particle size distribution becomes increasingly important in determining $\kappa$ (e.g., see CE98).
} 
that are relatively more massive.

Most recent analyses of the "autocorrelation lengths" (see Sec. 5) in the rings from Cassini data taken at face value seem to indicate that the typical size of particles are smaller (implying the opacity is actually higher) in the plateaus than away from them in the low- $\tau$ regions (Colwell et al., 2011; 2012). This could mean, for example, that the largest particles in the plateaus may be smaller than the largest particles outside of the plateaus, something that would likely affect the viscosity there as well. On the other hand, Marouf et al. (2012) find from direct inversion of the scattered Cassini RSS signal that the largest particles are much larger in the plateaus than in the surrounding $\mathrm{C}$ ring supporting Eq. (22). In this first paper, we merely explore some implications of our assumed opacity profile with the noted caveats until further analyses are conducted that resolve the apparent particle size paradox in the plateaus.

\subsubsection{Ring viscosity}

Apart from the angular momentum transport due to the ballistic transport process, the other non-Keplerian effect we consider in our model is viscous angular momentum transport. The inclusion of viscosity $\nu$ as a diffusive mechanism is key in counterbalancing the sharpening effects of ballistic transport, which can lead to unrealistic growth of (e.g., plateau) edges. Well prior to Cassini, the efforts to quantify ring viscosity both analytically (e.g., Goldreich and Tremaine, 1978; Araki and Tremaine, 1986) and numerically (e.g., Wisdom and Tremaine, 1988) assumed ensembles of identical spheres undergoing inelastic collisions while orbiting in the gravity field of the planet. A great advantage of these models, despite various simplifying assumptions, is that the description of the kinematic viscosity is expressible in a relatively simple, analytical form which Durisen and colleagues employed in their original structural evolution investigations.

For the bulk of the simulations presented here, we utilize a radially variable expression for the ring kinematic viscosity used by previous workers (Durisen et al., 1992; 1996; CE98) which is based on the work of Wisdom and Tremaine (1988), whose calculation of the viscosity includes both local and nonlocal contributions to the angular momentum flux. Durisen et al. (1992) provide an analytical fit to the numerical results of Wisdom and Tremaine (1988) for an ensemble of identical particles of size $r$

$$
\frac{3 \nu}{\Omega r^{2}}=2.5 \tau^{1.1}+\frac{1.7 \tau}{0.30+\tau^{2}}
$$

which has an accuracy of $\pm 5-10 \%$ for $0 \leq \tau \leq 2$ when compared with the numerical results. The first term on the RHS of Eq. (23) is due to the non-local shear stress, while the second term on the RHS is due to the local shear stress (e.g., see Schmidt et al., 2009) ${ }^{6}$. A suitable coefficient $\Omega r^{2} / 3$ was determined based on the results of simulations with two particle sizes (e.g., Salo, 1991) that showed that the overall spreading rate of particles is determined by the largest ones. For the $r^{-3}$ power law that we use, and our fiducial lower and upper bounds $r_{1}$ and $r_{2}$ (Sec. 2.2.2), the bulk of the mass and 1/3 of the optical depth

\footnotetext{
${ }^{6}$ Note that we have used the estimate for the velocity dispersion of the rings $c \sim 2 r \Omega$ appropriate for very flattened systems in the local shear stress term (e.g., Daisaka et al., 2001; Scmidt et al.., 2009)
} 
are due to particles with radii greater than $\bar{r}=64.6 \mathrm{~cm}$ (Durisen et al., 1992). Thus the expression for the kinematic viscosity in the BT code is

$$
\nu(R, \tau)=0.51\left(\frac{\bar{r}}{64.6 \mathrm{~cm}}\right)^{2}\left(\frac{1.55 \mathrm{R}_{S}}{R}\right)^{3 / 2}\left[1.5(\tau / 3)^{1.1}+\frac{(\tau / 3)}{0.30+(\tau / 3)^{2}}\right] \mathrm{cm}^{2} \mathrm{~s}^{-1}
$$

Durisen et al. (1992) found that a steady-state edge like that of the inner B ring can be sustained by a balance between BT, with a strongly prograde ejecta distribution, and viscous diffusion (using Eq. [24]). This finding was somewhat remarkable, considering the large optical depth contrasts between the low- and high- $\tau$ regions that characterize the inner $\mathrm{A}$ and $\mathrm{B}$ ring edges.

Choosing different values for $\bar{r}$, which effectively amounts to changing the particle size distribution upper and lower bounds, allows us to vary the magnitude of the viscosity in the code. We will refer to this variation in magnitude for specific model runs through a scaling factor $f_{\nu}=(\bar{r} / 64.6 \mathrm{~cm})^{2}$ which is unity for our fiducial parameters. Along the lines of the discussion in Sec. 2.2.6, we expect that we will be able to more reliably estimate $\bar{r}$ directly as we gain a better understanding of the ring opacity from continued analyses of the available Cassini occultation data.

We note that recent analyses of Cassini UVIS, VIMS and RSS occultation data have highlighted the "clump-and-gap" structure of the rings, presumably due to the presence of self-gravity wakes, with the majority of the ring mass possibly hiding in the opaque clumps, while the gaps contain a lower $\tau$ and perhaps smaller particles (e.g., Colwell et al., 2007;

Nicholson et al., 2008; Robbins et al., 2010). Consequently, a more robust model for the viscosity that takes into account the rings' wake structure should include the effects of mutual gravitational interactions (e.g., Hahn, 2008). We consider such a model in Appendix C. Although no wake activity has thus far been observed in the inner $\mathrm{B}$ ring and $\mathrm{C}$ ring (though see Colwell et al., 2007; 2009), we find that the $\mathrm{C}$ ring plateaus may marginally satisfy the condition for wake formation and we consider this briefly in Sec. 3.3.

\subsubsection{Ejecta yields and velocity distributions}

CE98 assumed the mass ejecta yield for primarily silicate material impacting icy particles to be in the range $Y \approx 10^{4}-10^{5}$. Given this range for the yield, the results of Lange and Ahrens (1987) imply a value of $Y=3 \times 10^{4}$ at an impact velocity of $14 \mathrm{~km} \mathrm{~s}^{-1}$, which is the value that CE98 adopted for their simulations. However, CE98 pointed out that their adopted values are scaled from experiments at lower impact velocities and refer to macroscopic projectiles hitting solid icy targets, rather than micrometeoroids impacting ring particles, where the regolith grains may typically be the same size or not much bigger than the impactor. Because ring particle bombardment continues to be a more complicated process than modeled in laboratory experiments, we choose to use the yield as a variable parameter with a range from $\sim 10^{4}-10^{6}$, which covers hard to soft, fluffy or porous targets.

Our ejecta speeds are parametrized by the dimensionless variable $x$ and the ejecta velocity distribution is given by a pure power law 


$$
f(x)= \begin{cases}x^{-n} & x_{b} \leq x \leq x_{t} \\ 0 & \text { otherwise }\end{cases}
$$

where $f(x) d x$ describes the fraction of ejecta mass having speeds in the interval $x$ to $x+d x$, and $x_{b}, x_{t}$ are the adjustable lower and upper bounds of the ejecta distribution. Durisen et al. (1992) and CE98 adopted a value of $n=13 / 4$ which was a fit to the high-end ejecta velocities in hard target laboratory data for solid basalt (e.g., see Burns et al., 1984). If icy ring particles act more like sand, then a slightly less steep slope may be more appropriate. Here, we adopt $n=3$ as our fiducial value. We explore different values of $n$ in Sec. 3.1.

In this paper, we only model a pure power law for $f(x)^{7}$. Because of the improved computational ability of our parallelized BT code, we can go beyond Durisen et al. (1992) and simulate large radial regions over long periods of time while simultaneously being able to resolve structure for very small $x_{b}$. Moreover, the BT code can readily be altered to adopt velocity distributions which have a more complex behavior. In future work, we will consider velocity distributions that go beyond simple power laws and include isotropic and retrograde (relative to Keplerian) dominated impact ejecta distributions due to disruptive impacts (see discussion in Sec. 5). These may require that we rerun the ejecta distribution calculations of CD90 with altogether different sets of conditions.

\section{Ballistic Transport Simulations}

\subsection{Comparison to Previous Work}

In this section, we present some tests of the numerical code. A natural starting point for demonstrating the new code's utility is to reproduce results in earlier papers. Durisen et al. (1992) modeled the relatively sharp B ring edge and its transition into a low constant optical depth $\mathrm{C}$ ring and demonstrated that the $\mathrm{B}$ ring inner edge (and presumably also the similar inner A ring edge) can be maintained at its presently observed width through a balance between viscosity and ballistic transport with a prograde-biased ejecta velocity distribution. Durisen and colleagues explored a range of impact yields and ejecta speed distributions $f(x)$, with different choices for the lower bound $x_{b}$, to find a range over which the $\mathrm{B}$ ring edge could be maintained over long time scales. Here, we focus on a few specific cases choosing the same parameter values as Durisen et al. (1992).

Durisen et al. (1992) scaled their models using a relationship between the surface mass density and optical depth at $R=1.52 \mathrm{R}_{S}$ such that $\sigma=\sigma(\tau=1) \tau$ where $\sigma(\tau=1)=96$ $\mathrm{g} \mathrm{cm}^{-2}$. This scaling then represents a constant opacity model for the rings with an effective value $\kappa=\tau / \sigma=0.104 \mathrm{~cm}^{2} \mathrm{~g}^{-1}$, and, along with the assumption that the ring particles everywhere can be described by an $r^{-3}$ power law with our assumed $r_{1}$ and $r_{2}$, is consistent

\footnotetext{
${ }^{7}$ Durisen et al. (1992) looked at pure power laws as we utilize here, but also looked at a power law with a "knee" which was considered more applicable to model the hard target, higher-end speed distribution. The main difference between the two distributions that Durisen and colleagues found was that the pure power law required somewhat higher yields to dominate viscous transport and produced a steeper steady-state edge.
} 
with mostly icy ring particles with density $1.12 \mathrm{~g} \mathrm{~cm}^{-2}$. The initial condition for the B ring edge models has the optical depth distribution

$$
\tau(R, 0)=0.050+\frac{0.950}{e^{\left(1.52 \mathrm{R}_{S}-R\right) / D}+1},
$$

where the parameter $D=0.0015 \mathrm{R}_{S}$ gives an edge width of $\sim 90 \mathrm{~km}$, comparable to what is observed.

In Figure 2, we show a B ring inner edge model with $Y=3 \times 10^{5}$, a power-law ejecta speed distribution with a lower bound $x_{b}=2 \times 10^{-4}$, and a kinematic viscosity with weighting factor $f_{\nu}=1$, matching parameters in Fig. 10a, Durisen et al. (1992). For these choices of parameters, the gross erosion time is around $4.5 \times 10^{4}$ years. The simulation uses a time step of $\Delta t=0.0025 \mathrm{t}_{G}$, and the entire run, which corresponds to a physical timescale of $4.5 \times 10^{6}$ years, took 35 minutes of parallel CPU clock time. The black curves correspond to selected times during the evolution of the ring structure, while red curves of the same style are the corresponding fractional mass of pollutant. Over the first ten gross erosion times or so, the ring edge steepens to a roughly steady-state slope. A ramp at the base of the edge starts to develop between 9 and $35 \mathrm{t}_{G}$ and becomes quite prominent by the end of the run at $99 t_{G}$. The ramp's growth is produced by a small residual between direct mass exchanges and "indirect" radial drifts due to ballistic transport in this region, which is essentially independent of the ring viscosity (i.e., $\left|v_{\mathrm{R}}^{\text {ball }}\right| \gg\left|v_{\mathrm{R}}^{\mathrm{visc}}\right|$ ) there (Durisen et al., 1992). The undulatory structure (Durisen, 1995) that appears in the B ring for higher yields (see Fig. 4) is suppressed by viscosity for this value of $Y$. Also observed further inwards and shown in more detail in Figure 3 are undulations that emanate from the base of the ramp. These undulations have long wavelengths and may be being forced by the growing ramp structure which itself is quite broad. Alternatively, these undulations may come as a result of the linear BTI which favors regions of intermediate $\tau$ (Durisen 1995; Latter et al., 2012; $2014 \mathrm{a}, \mathrm{b})$. As the ramp grows in width, so too does the wavelength of the low optical depth region undulations. These undulations are not so easily suppressed because the viscosity is much weaker here than in the B ring (cf. Eq. [24]).

Also plotted in Figures 2 and 3 (red curves) are the fractional pollutant mass in the rings for the selected evolutionary times (see Sec. 2.2.3). Initially, the ring begins with no extrinsic pollutants anywhere $\left(f_{\mathrm{e}}(R, 0)=0\right)$. At earlier times, the fractional mass differences between the high optical depth $\mathrm{B}$ ring and low optical depth $\mathrm{C}$ ring are quite pronounced, with a sharp transition across the $\mathrm{B}$ ring inner edge. As the simulation evolves, the differences in composition between the regions retain a nearly constant ratio (roughly between $1.49 \mathrm{R}_{S}$ and $1.54 \mathrm{R}_{S}$ ), but the sharpness of the transition smears out over time due to advective effects, an effect found by CE98 in their compositional evolutions. Less polluted material from the higher mass density B ring drifts inwards, displacing more polluted material inwards of the boundary, as seen in observations (Cooke et al., 1991; Estrada and Cuzzi, 1996).

The compositional contrast between low- and high- $\tau$ regions depends on the opacity contrast between the regions. The CE98 profile for the opacity given in Eq. (21) would produce higher contrast than shown in Figures 2 and 3, but its simple form cannot readily be applied to the structurally evolving models because it relies on a fixed position for the inner $\mathrm{B}$ ring edge. In a structurally evolving model, one would have to redefine $R_{1}$ and $R_{2}$ 
as the edge evolved, which may occur either by drifting radially inward, and/or by smearing due to viscosity. This problem is not encountered in the opacity model given by Eq. (22) because $\kappa$ depends on the value of $\tau$, not on $R$. In Figure 3 the most notable thing is that the relative radial position of the decrease in fractional mass begins further radially inward in the ring with time. This is probably due to two effects: the rings are brightening further inwards as time goes on as brighter material drifts in from the edge and darkening is occurring less quickly as the ramp grows in mass (and radial extent) relative to regions inside of it.

In Figure 4, we reproduce the results of a compilation of simulations over $104 \mathrm{t}_{G}$ for the B ring edge model for various combinations of $Y$ and $x_{b}$ by Durisen et al. (1992, their Fig. 15). The dashed curve corresponds to the case for $Y=3 \times 10^{5}$ presented previously (for $99 \mathrm{t}_{G}$ ) with the run extended for five more gross erosion times. The dotted and solid curves represent cases with yields of $Y=1 \times 10^{6}$ and $2 \times 10^{6}$, respectively, and a lower bound in the ejecta velocity distribution of $x_{b}=1 \times 10^{-4}$ which corresponds to a velocity of $\sim 2$ $\mathrm{m} \mathrm{s}^{-1}$. These runs also have the number of bins increased to 1500 from the 1000 used in the $Y=3 \times 10^{5}$ case, as was done in Durisen et al. (1992). Furthermore, the width parameter (see Eq. [26]) is chosen to be much narrower, $D=0.00075$ in order to account for the finer grid being used. Also plotted in Fig. 4 is the Voyager PPS optical depth profile (opening angle $B \sim 28^{\circ}$ ) for comparison ${ }^{8}$. The main difference between the two latter cases is that the $Y=1 \times 10^{6}$ case achieves a steady-state sharpness with an absence of high- $\tau$ undulations, while increasing the yield by only a factor of two quite readily produces nonlinear growth of undulations in the inner $\mathrm{B}$ ring. The corresponding red curves are the fractional masses for each run. The highest yield case has the least amount of darkening which may seem counter-intuitive, but this is because the actual exposure time is much shorter for the same value of $t_{\mathrm{G}}$. For the $Y=10^{6}$ and $Y=2 \times 10^{6}$ runs, the physical time scale for exposure is $1.4 \times 10^{6}$ and $6.8 \times 10^{5}$ years, respectively.

From the standpoint of structural evolution, we find no significant differences between our simulations and those of Durisen et al. (1992). The differences that do appear upon close inspection may be due mostly to order $x$ errors which would be most prominent near the sharp edge. We note that the simulations of Durisen et al. $(1989 ; 1992)$ used an erroneous expression for the angular momentum of an ejectum $h$ (Eq. [22], Durisen et al., 1989) that propagated to the expression for the function $A(x, \theta, \phi)$ (see Sec. 2.2.5) used in their code (Durisen et al., 1996). Here we use the corrected expression for $A$ that is derived using the appropriate $h$. Furthermore, we ran the higher yield cases at a smaller time step than used by Durisen et al. $\left(\Delta t=0.0025 \mathrm{t}_{G}\right)$. Our runs were done with a time step of $\Delta t=0.0005$ and $0.0002 \mathrm{t}_{G}$ for the $Y=10^{6}$ and $Y=2 \times 10^{6}$ cases, respectively. As an example of code execution time, the $Y=2 \times 10^{6}$ case took $\sim 300-400$ minutes of dedicated parallel CPU clock time to complete.

In Figure 5, we ran models with the same parameter choices as in Fig. 4, except that, instead of constant opacity, we use the variable opacity from Eq. (22), in which the particles are assumed to be larger in the higher- $\tau$ regions. The magnitude of the opacity in the $\mathrm{C}$ ring and $\mathrm{B}$ ring is comparable to what was used in CE98 (specifically, $\kappa(\tau=0.05)=0.05 \mathrm{~cm}^{2}$

\footnotetext{
${ }^{8}$ The Voyager PPS optical depth was used by Durisen and colleagues, and CE98 in their work. In this paper, we also use the higher-resolution Cassini UVIS $\alpha$-Arae occultation data as our reference $\tau$ when modeling real structure.
} 
$\left.\mathrm{g}^{-1}, \kappa(\tau=1)=0.009 \mathrm{~cm}^{2} \mathrm{~g}^{-1}\right)$. The higher $\mathrm{C}$ ring opacity translates to more mass in the plateaus compared to constant $\kappa$, but about a factor of five less mass overall in the $\mathrm{C}$ ring than in the constant opacity model, because there is much less mass outside of the plateaus. The inner B ring mass remains comparable.

The greater contrast in mass density between in the inner B ring and the non-plateau regions of the $\mathrm{C}$ ring leads to two clear differences. First, the difference in darkening between the $\mathrm{C}$ ring and $\mathrm{B}$ ring is much more pronounced, as one would expect for the larger mass density contrast. In other words, the $\mathrm{C}$ ring darkens much more quickly relative to the $\mathrm{B}$ ring for a given exposure time. The greater contrast in mass also affects the growth of undulations in the inner $\mathrm{B}$ ring. Whereas in Fig. 4 we see that the $Y=2 \times 10^{6}$ case produces large undulations, which continue to grow to large amplitude at longer times, the introduction of the variable opacity model mutes the growth of these undulations. One reason for this, at least in part, has to do with the fact that the lower surface mass density in the $\mathrm{C}$ ring decreases the amount of material BT throws into the B ring. However, longer term runs not shown here do not lead to further growth of the undulations as they did in the constant opacity case; instead they remain roughly constant in amplitude with time, somewhat like what is seen in Fig. 8. Thus, a second reason may be that the variable opacity itself acts as a damping mechanism to regulate growth.

This effect can be seen more clearly by comparing $\partial \tau / \partial t$ for the two opacity models as shown in Figures 6 and 7 (cf. Durisen et al., 1992; 1996). In these figures, the top panel shows the individual contributions to the change in $\tau$ with time due to the direct mass exchanges between neighboring ring regions (dotted curve), and due to the BT (indirect) advective term. The bottom panel shows a comparison between the combined BT effects, the viscous effects, and their sum shown on an expanded scale. The net change (all effects included) is given by the red curve. Outwards of $1.52 \mathrm{R}_{S}$, which marks the inner B ring edge, the BT effects are weak at earlier times and generally are balanced out by viscosity. As time progresses and the effects of exposure begin to accumulate, the competition between BT and viscosity in the inner $\mathrm{B}$ ring become more and more imbalanced, and a net growth begins to occur leading to the undulations we see in Fig. 4. It should be noted that the undulations seen in the $\mathrm{B}$ ring, much like the in $\mathrm{C}$ ring, could be arising due to the BTI with the growth rates being small because the instability is near marginality (see Latter et al., 2012).

Even though the viscosity is strongest inside the B ring edge, the perturbed growth is more or less "stationary", which allows for the undulations to grow "in place". This is in contrast to the $\mathrm{C}$ ring where, although the magnitude of the variation in $\partial \tau / \partial t$ is greater than what is seen in the inner B ring, the variations are transient (i.e., not stationary) in nature and tend to somewhat average out. This is consistent with recent work by Latter et al. (2014a,b) who show that B ring wavetrains have very small wave speeds, whereas the $\mathrm{C}$ ring waves propogate much faster. Finally, in this region, the viscosity has little effect so the solid red and black curves overlap. On the other hand, and as also seen in Fig. 5, the variable opacity model in Fig. 7 is characterized by much smaller net effects away from the sharp edge. 


\subsection{Extending Previous Work and Global Simulations}

\subsubsection{Long Term Stability of the B ring Inner Edge}

In Figure 8, we have simulated the inner B ring edge with constant opacity using a yield of $Y=1.5 \times 10^{6}$ with $x_{b}=10^{-4}$, and $f_{\nu}=1$ for a timescale of $800 \mathrm{t}_{G}$. This run is an extension of Durisen et al. (1992, their Fig. 15) with the exception that ring torques and mass loading are included. With these parameter choices, the absolute time scale of the run is $\sim 7 \times 10^{6}$ years. We have plotted several curves in $100 t_{G}$ intervals to demonstrate quite clearly how BT is able to maintain the sharpness of the inner B ring edge over long periods of time, a result that was implied by Durisen and colleagues, but not shown explicitly in their work. The development of this steady-state edge due to a balance between viscous spreading and the sharpening effects of BT for the initially narrow edge and prograde ejecta distribution we use is dominated by the direct mass effect (Durisen et al., 1992), as shown in Fig. 6. Viscosity, however, does not play a role in the formation of the ramp which is due to advection overbalancing the direct mass term (see Appendix B of Durisen et al., 1992).

Although the overall slope of the ramp region in this simulation appears to be consistent with the linear ramp seen in the observations (dash-dotted curve), a "hump" structure forms there between $1.51-1.52 \mathrm{R}_{S}$. At this time, it is not clear what the cause of this feature is. A possible explanation is that the hump may be a manisfestation of the BTI, but operating against a very nonuniform background where it is less well understood. We consider this as a topic worthy of further study. In the inner B ring, the undulations appear to be approaching a steady-state away from the edge for this choice of parameters. Recall that there are none for $Y=10^{6}$ and that they grow to large amplitude for $Y=2 \times 10^{6}$. This result highlights the sensitivity of the simulations to the choice of parameters for the constant opacity case, in particular. As we have demonstrated previously, the variable opacity model is less sensitive to the parameter choices and thus may allow for a more robust parameter space to work with, although we again emphasize that the $\kappa(\tau)$ we use is heuristic with significant caveats (Sec. 2.2.6). We have done the complementary case with a variable opacity for the same set of parameters. We show only the profile after $800 \mathrm{t}_{G}$ (red curve, Fig. 8). The main difference is that the inner B ring edge slowly spreads, which implies that a slightly larger yield will be needed to maintain it. We also find that even though the $\mathrm{C}$ ring has five times lower $\sigma$ overall than the constant $\kappa$ case, the hump structure in the ramp is slightly lower in magnitude and the wavelength of the $\mathrm{C}$ ring undulations are longer. The $\mathrm{C}$ ring optical depth is also larger due to the spreading of the $\mathrm{B}$ ring inner edge. Lastly, the undulations in the inner B ring are muted, but this may also be due to the lack of a steady-state edge in addition to fewer ejecta being delivered from the $\mathrm{C}$ ring to the inner $\mathrm{B}$ ring.

\subsubsection{Steepness of the Ejecta Velocity Distribution}

As shown in Figure 9 for a yield of $Y=3 \times 10^{5}, x_{b}=10^{-4}$ and $f_{\nu}=0.125$, we have run a set of simulations varying the steepness $n$ of the ejecta velocity distribution. The inner B ring is modeled with a larger $\tau \sim 1.5$, consistent with the $\alpha$-Arae UVIS optical depth profile, dotted curve, because the simulation for $n=2$ (blue curve) leads to the inner edge becoming quite sharp and the ensuing undulations in the inner $\mathrm{B}$ ring grow to large amplitude for the 
case where $\tau \sim 1$ in the $\mathrm{B}$ ring. A model for the fiducial choice of $n=3$ is given by the black curve. There are few things of note that we can identify in these examples. For higher $n$ a "notch" appears in the inner B ring edge somewhat similar to what is seen in the data. We also see that steeper velocity distributions associated with larger $n$ values lead to a gradual "spilling over" of material from the inner B ring edge, even as the slope of the edge closer to its base remains similar to its initial steepness. It is possible to interpret the occultation data as showing multiple notches in the edge, with a more gradual drop off in $\tau$ (dotted curve) similar to what is seen for the $n=3-5$ curves. The spreading of the edge occurs because the steeper velocity distributions concentrate more material at the smallest $x$ thus weakening the larger-scale effects of BT relative to viscosity, particularly at the higher $\tau$.

We suggest that the notches seen in these simulations are related to the BTI, but under very nonuniform conditions. The notches appear to be more pronounced for steeper speed distributions. The reason for this is that higher $n$ values places more ejecta in lower $x$-values which reduces the ejecta contribution to ramp production. Because the ramp is less prominent for higher $n$, the ability for BT to overwhelm viscosity at the smallest $x$-scales is increased (similar to increasing the $\mu$ parameter, Latter et al., 2012; 2014a,b) which allows for BTI-like behavior. Figure 9 further suggests that the BTI-like growth of waves could be producing the hump structure seen in Fig. 8. The hump is at an intermediate length scale because viscosity is able to control the smaller scale BTI-like waves for $n=3$.

What may be more interesting is that lower values of $n$ lead to a larger and better formed ramp, because as $n$ decreases, more ejecta is shifted to intermediate and higher values of $x$ so that BT cannot compete well at small scales. This is also the reason why the inner edge sharpens further. We note that in Eq. (15) of Durisen et al. (1992), they also predicted a linear ramp for a value of $n=2$. In their analytical model for ramp production, the first term of Eq. (15) predicts what is seen in Fig. 9 quite well, but the second term becomes divergent very close to the inner edge, which we do not see as-of-yet over the simulation time of $100 \mathrm{t}_{G}$. In any case, the real ring edge does not look like the $n=2$ profile, but more like a hybrid of low - and high $-n$ results. That is, the real inner edge seems to exhibit features characteristic of multiple ejecta distributions with different $n$-values at different $\tau$ 's and $x$-values, and with prograde and retrograde symmetries. This strongly hints at the considerable complexity involved in the ejecta distribution function which requires further, in-depth study.

\subsubsection{Hidden Mass in the B ring}

The surface mass density in the opaque regions of the B ring is not known, but it has been pointed out that, if the $\mathrm{B}$ ring is an order of magnitude more massive than the current estimate, then it could more easily resist becoming polluted and darkened, and perhaps be as old as the Solar System (Esposito, 2008; Charnoz et al., 2009; Robbins et al., 2010). To explore this possibility, we show a low-resolution simulation of two hypothetical B ring structures over $100 \mathrm{t}_{G}$ using a yield of $Y=3 \times 10^{5}, x_{b}=10^{-4}$ and variable opacity in Figure 10. We have chosen to use the kinematic viscosity, but modified it in such a way that the viscosity is able to maintain both the inner and outer $\mathrm{B}$ ring edges. The reason for this is that straight application of the wake viscosity (Eq. [C-1], Appendix C) to the B ring requires 
unrealistic yields to prevent spreading of the inner edge and smearing out of structure even in the high $-\sigma$ regions (see Sec. 2.2.7). In order to maintain the sharpness of both the inner and outer $\mathrm{B}$ ring edges, we have incorporated a simple linear increase in $\nu(R)$ outwards of $1.6 \mathrm{R}_{S}$, up to a factor of two larger $\nu$ than what it would otherwise have been at the outer $\mathrm{B}$ ring edge. The dashed black and red curves are the structure and fractional mass of pollutant, respectively, for a "lower bound" of the B ring mass estimated by Robbins et al. (2010, $\sigma \sim 400 \mathrm{~g} \mathrm{~cm}^{-2}$ in the core) which is already larger than traditional "Mimas" mass estimates indicated by the solid curves. The corresponding "high mass" model $(\sigma \sim 1000$ $\mathrm{g} \mathrm{cm}^{-2}$ in the core), as suggested by Esposito (2008), is shown by the dotted curves. These two cases produce a measurable difference in the amount of darkening that would occur in the $\mathrm{B}$ ring core, both between the traditional mass model (solid curves), and with each other, as well as qualitative differences in composition between the "troughs" in fractional mass compared with the rest of the $\mathrm{B}$ ring. Likewise the difference between the $\mathrm{B}$ ring core and the $\mathrm{C}$ ring (and Cassini division) emphasizes the notion that if the $\mathrm{B}$ ring is indeed more massive, then the low optical depth regions would have become overpolluted over the age of the Solar System, unless they are somehow either regenerated and/or replenished with bright material. In future work, we will be addressing these issues in further detail.

\subsection{Simulations of Observed Structure in the $\mathrm{C}$ ring}

The simulations presented in Section 3.1, and those of Durisen and colleagues, used as initial conditions simple local models for ring initial structure, namely a moderately sharp edge bracketed by low and high constant optical depths emphasizing inner edges such as those of the B and A ring. Simulations such as these are useful for determining how some of the structure we see at or near these inner edges may be produced by BT. Here we explore simulations in which the initial conditions consist of the observed optical depth profiles for parts of the $\mathrm{C}$ ring in an effort to understand what role BT may play in maintaining observed structures such as plateaus over long periods of time. The Cassini UVIS $\alpha$-Arae occultation (Colwell, priv comm) optical depth profile we use as our initial $\tau$ has been modestly smoothed using a three-point boxcar routine. All simulations in this section utilize the pure power-law velocity distribution with $n=3$ described in Sec. 2.2 .8 with $x_{b}=1 \times 10^{-4}$ and $x_{t}=4 \times 10^{-3}$, the kinematic viscosity (Sec. 2.2.7) with $f_{\nu}=1$, and our variable opacity model (Sec. 2.2.6), unless otherwise noted.

In Figure 11 we show a suite of simulations for yields of $Y=10^{4}$ (solid curves), $10^{5}$ (dotted curves) and $10^{6}$ (dashed curves) for a period of $2 \mathrm{t}_{G}$ over the bulk of the $\mathrm{C}$ ring and inner $\mathrm{B}$ ring. The radial resolution is $\sim 6.5 \mathrm{~km}$ per bin. The gross erosion time scales associated with these yields derived from Eq. (5) are $t_{\mathrm{G}}=1.4 \times 10^{4}-10^{6}$ years with the longest time scale corresponding to $Y=10^{4}$, but as mentioned in Sec. 2.2.1 this timescale is normalized to $\tau=1$. A representative range of $\tau$ for the plateaus in the $\mathrm{C}$ ring from the optical depth profile we utilize (blue curve) is $\tau \sim 0.5-0.25$. Using our variable opacity model, these values then imply that the gross erosion times are $\sim 2-6$ times shorter than the values given above for the lower $\tau$ regions.

In Fig. 11, the black curves correspond to the structural evolution, while the red curves correspond to the composition. It is evident that, for the parameter set chosen, the effects 
due to BT for a relatively small yield of $10^{4}$ are insufficient to maintain any structure against spreading by our fiducial kinematic viscosity. Inside $\sim 1.5 \mathrm{R}_{S}$, the outer edges of the plateaus, which should be maintained if the prograde ejecta yield were large enough, smear out as quickly as the inner edges. Also, outside of this location, the integrity of the B ring inner edge is lost and smears almost as vigorously as the lower optical depth plateaus. This behavior also seems to characterize a yield of $Y=10^{5}$, although less so. Given that the outer edges of the plateaus are becoming less sharp, it seems that a longer term simulation would lead to smearing out of most of the structure here as well. On the other hand, a yield of $10^{6}$ actually leads to a BT influence that is too large for the plateau regions, because the prograde ejecta distribution sharpens the plateau outer edges more than is observed. The observed plateaus outside of $R=1.46 \mathrm{R}_{S}$ have a much more gradual outward increase in $\tau$ (similar in slope to the ramp itself). If $Y$ is really this high, stronger advective and/or viscosity effects may be needed to prevent as much pileup of material at outer edges as the simulations show. However, the great sensitivity of the effect to $Y$ suggests that other properties may come into play. For instance, maybe there is a feedback on $Y$ itself, as the particle properties change due to structural evolution.

Also shown in Fig. 11 are the corresponding compositional profiles for $2 \mathrm{t}_{G}$. We note that all three compositional evolutions have been scaled to the $Y=10^{4}$ values for the fractional pollutant mass $f_{\mathrm{e}}$ for the purpose of comparison. The calculated amount of pollutant in the higher yield simulations is 10 or 100 times less for $Y=10^{5}$ or $10^{6}$, respectively, by the definition of $t_{\mathrm{G}}$ (Eq. 5), because the amount of extrinsic material that has hit the rings is also 10 and 100 times less in these cases. Much like the structural evolution, the evolution of fractional mass of pollutant for a yield of $10^{4}$ leads to a profile of composition that is quite diffuse, and the features drift inwards as they smear. The compositional profiles for $Y=10^{5}$ and $10^{6}$ look remarkably similar even though their structural profiles do not. This suggests that compositional gradients associated with higher-optical depth structure embedded in a lower optical depth background have a more gradual radial variation than those in optical depth and, moreover, are insensitive to fine-scale structural details. In all three cases, the compositional transition between the inner $\mathrm{B}$ ring to the $\mathrm{C}$ ring is broad, even though the transition in brightness and optical depth between these ring regions is abrupt (Estrada and Cuzzi, 1996; Estrada et al., 2003; Cuzzi et al., 2002; 2009). This is consistent with the earlier finding by CE98, using fixed structure, that a broad observed color transition across an abrupt optical depth transition was produced by ejecta exchanges. The results of Fig. 11 can be thought of as a generalization of this B-C boundary effect in that, at all abrupt plateau edges, the pollution profile is smoother than $\tau(R)$ and insensitive to finescale structure. CE98 ascribed the B-C compositional gradient to inward advection of less polluted B ring material.

These simulations suggest that a proper balance between the sharpening effects of BT due to a prograde ejecta distribution versus the broadening effects of viscosity could possibly maintain outer plateau edges at their current level of steepness over long periods of time, but in a rather parameter-sensitive way. The fact that the inner edges of the plateaus do not sharpen as strongly in the current simulations argues for a significant component of the ejecta distribution to be retrograde relative to Keplerian, perhaps due to disruptive impacts. Simply from symmetry arguments, this would have the effect of sharpening the inner edges 
because a significant component of radial drift could be outwards (from inside the plateau). A retrograde component to the ejecta distribution may also play a role in ramp formation (Durisen et al., 1992), potentially leading to suppression of the hump that we see forming in the ramp in our simulations.

The observed optical depth profile for the rings (Fig. 1) may indicate that retrograde ejecta play a significant role in the $\mathrm{C}$ ring and are even consistent with this role increasing inwards (Sec. 1). In future work, we will formally include different ejecta distributions (see Sec. 5 for a discussion of retrograde ejecta). Durisen and colleagues also found high yields to be necessary to maintain the inner B ring inner edge, even assuming only kinematic viscosity (see also Fig. 8). The explanation for how BT sharpens an inner edge, where a large optical depth contrast exists between the low- $\tau$ and high- $\tau$ regions, is complicated, even in linear analyses that simplify the process, yet still preserve the essential features (see Durisen et al., 1992, for a full discussion). The important thing to note here is that the inner $\mathrm{B}$ ring edge, and by analogy the inner A ring edge, both satisfy the conditions for optical depth contrast that lead to maintaining a sharp, steady-state inner edge, whereas the $\mathrm{C}$ ring plateau inner edge optical depth contrasts do not. Thus having a retrograde component to the ejecta distribution may be essential for maintaining the $\mathrm{C}$ ring structure we observe. This component, if due to disruptive impacts, may have a very different velocity and angular distribution from the cratering impact ejecta modeled in this paper.

In Figure 12 we show an example of the effects of kinematic viscosity in maintaining ring structure for different choices of the ejecta yield. The justification for this parameter study is that the bulk of the optical depth may be due to particles of size $\bar{r}$, which are different from our fiducial choice $\left(f_{\nu}=1\right.$, Sec. 2.2.7), as might come about, e.g., if the size distribution in the rings were narrower or broader than we assume (Colwell et al., 2009; Marouf et al., 2012). This may be particularly true within the plateaus (see discussion, Sec. 5). Figure 12 shows three different simulations of the plateau features centered at $R=1.465 \mathrm{R}_{S}$ using yields of $10^{4}$ (black curve), $10^{5}$ (red curve) and $10^{6}$ (blue curve) over a period of $2 \mathrm{t}_{G}$ for three different choices of $f_{\nu}$ such that $Y / f_{\nu}=10^{5}$ (see below). This means the red curve corresponds to the $Y=10^{5}$ curves in Fig. 11. The initial optical depth profile is given by the dotted curve. The model runs with $f_{\nu}=0.1$ and $f_{\nu}=10$ translate to $\bar{r}=20.4$ and 204 $\mathrm{cm}$, respectively. The characteristic optical depth for these plateau features is $\tau \sim 0.25-0.3$ so that the gross erosion times are about $\sim 5$ times shorter than the timescales given for Fig. 11.

One point that the parameter choices in these simulations are meant to demonstrate is that their exists a degree of scaling between $f_{\nu}$ and $Y$ which can produce the same set of results through variation of these parameters ( $c f$. Latter et al., 2012, their $\mu$ parameter). This was pointed out by Durisen and colleagues who conflated $Y$ and $\nu$ into an "effective yield" in their calculations. As long as the functional forms of $\tau(R, 0), \nu(R, \tau), f(x)$, and $\sigma(\tau)$ remain fixed, each simulation represents an infinite family of solutions (Durisen et al., 1992). The reason for this is that, if these functional forms are fixed, the changes with time depend only on a dimensionless ratio of the ballistic transport time scale $t_{\mathrm{G}}$, to the viscous timescale $t_{\nu}=L^{2} / 3 \nu$ where $L$ is the length scale over which viscosity acts. With the variable opacity model, however, it is not clear that this scaling invariance will hold over very long time scales. Figure 12 indicates that it is still valid over short time scales. 
Thus in all three cases, even though the absolute time scales between them range by two orders of magnitude between $Y=10^{4}-10^{6}$, the profiles look similar with the outer edge slopes being comparable in steepness, while the slope within the outer part of the plateau itself remains similar to the initial one. The fiducial value of $f_{\nu}=1$ and a yield of $10^{5}$ seems to be adequate in maintaining the sharpness of the outer edge, although some degradation of the edge, and more notably the decrease in the optical depth of the outermost plateau at $\sim 1.486 \mathrm{R}_{S}$, would suggest at slightly higher value for $Y$. However, for a yield of $10^{4}$ and the fiducial $f_{\nu}$, the BT mechanism is too weak to maintain structure against viscous spreading, and all structural features are smoothed out in only $2 \mathrm{t}_{G}$. On the other hand, a yield of $10^{6}$ and $f_{\nu}=1$ leads to the largest pileup of material at the outer edge as BT dominates the viscosity producing a spike in the amplitude of $\tau$ there (see both the $Y=10^{4}$ and $10^{6}$ cases in Fig. 11). Although not shown here, for this high a yield structure begins to form within the plateau itself; yet, this same combination of parameters is effective at maintaining the sharpness of the (larger optical depth contrast) inner B ring edge. This may imply that particle size distributions and/or particle properties (which may affect yields) are different for the $\mathrm{C}$ ring plateaus compared to the inner $\mathrm{B}$ ring. The similarity of these simulations suggests that the wide range in possible impact yields could be accomodated for if the dominant particle size can be different from our assumed value $(\bar{r}=64.6 \mathrm{~cm})$. More work will be required to determine particle properties in the plateaus before we can draw any definitive conclusions.

The main difference between these simulations is in how much the inner edges have diffused, with radial diffusion being more evident in the lower yield case where the "real" time is longer (for $Y=10^{4}, 2 \mathrm{t}_{G}=2.7 \times 10^{6}$ years). If we were to run these simulations (as well as those in Fig. 11) for longer times $t>2 \mathrm{t}_{G}$, the inner edges would smear out for any value of $f_{\nu}$ because there is currently no mechanism in the code to sharpen inner edges of low-to-moderate optical depth. The ejecta distribution we employ at present is prograde so that BT can only sharpen outer edges for these low optical depth features (see Sec. 5).

In Figure 13, we vary the lower bound $x_{b}$ of the ejecta velocity distribution to illustrate the sensitivity of the rings' structural and compositional evolution to this parameter for $Y=10^{5}$ and a value of $f_{\nu}=0.3$ which correpsonds to a dominant particle size $\bar{r}=35.4$ $\mathrm{cm}$. We chose $x_{t}=4 \times 10^{-3}$ such that the maximum ejecta velocity $v_{\mathrm{ej}}=100 \mathrm{~m} \mathrm{~s}^{-1}$ at $1.5 \mathrm{R}_{S}$. The structural evolution over $2 \mathrm{t}_{G}$ is plotted in the upper panel for $x_{b}=10^{-4}$ (black curve), $x_{b}=5 \times 10^{-5}$ (blue curve), $2 \times 10^{-5}$ (red curve) and $x_{b}=2 \times 10^{-4}$ (green curve) which correspond to lower bound velocities of $2,1,0.4$, and $4 \mathrm{~m} \mathrm{~s}^{-1}$, respectively. In some ways, lowering $x_{b}$ seems to have a similar "softening" effect as increasing the viscosity, in that the amplitude of the plateau outer edges and the amount of structure within the plateaus themselves decrease with smaller $x_{b}$. However, while the amplitude does decrease, the sharpness of the plateau outermost edges only decreases slightly with smaller $x_{b}$ for this choice of the viscosity, although the location of the edge is systematically different for the three cases. This result is straightforward to interpret. For an ejecta velocity distribution with a slope of $n=3$, most of the mass is ejected at $x_{b}$ and falls off steeply at larger $x$. The throw distance of this low-speed ejecta is small, meaning that the angular momentum that it carries is being deposited at locations whose angular momenta are not too dissimilar, and the smaller mismatch produces smaller advective radial velocities. 
The compositional evolution associated with this simulation is presented in the lower panel. The fractional mass of pollutant $f_{\mathrm{e}}$ is plotted with the initial optical depth profile (dotted curve) shown for reference. The most interesting thing to note is that the lowest $x_{b}$ case has the most pronounced compositional variation, whereas the opposite is true in the structural evolution plot. The interpretation here is that the pollution transport is much more localized for lower ejecta velocity. This is illustrated by noting the amount of the pollution in the low $-\tau$ regions between the plateaus is larger because brighter material from the high $-\tau$ regions has not had enough time to advect into the low $-\tau$ regions. The largest $x_{b}$ (green curve) emphasizes how a large lower bound can lead to significant pollution transport even though the sharpening of the outer edge structure is the largest in this case. A more localized transport due to the lower ejecta velocities may allow the plateaus to better retain their compositional identity relative to surrounding regions for longer periods of time.

Finally, in Figure 14 we use a combined viscosity model that replaces our usual kinematic viscosity (Eq. [24]) by the Daisaka et al. viscosity (Eq. [C-1]) when the condition for wake formation (Eq. [C-2]) is satisfied in the plateaus (see Appendix C). Three curves are shown for $Y=10^{6}$ that focus on the plateaus just inside the ramp centered at $1.495 \mathrm{R}_{S}$. The black curve corresponds to the simulation from Fig. 11 for the same yield. We have already indicated that such a large yield for the plateaus leads to BT that overpowers the kinematic viscosity for the nominal $f_{\nu}=1$, and stability requires a larger particle size $\bar{r}$ to dominate the optical depth (cf. Fig. 12, $f_{\nu}=10$ ). On the other hand, the red and blue curves, which correspond to ring particle densities $\rho$ of 0.5 and $0.9 \mathrm{~g} \mathrm{~cm}^{-3}$, respectively, suggest that wake formation in the plateaus may increase viscosity enough to suppress the tendency of BT to produce large amplitude "spiky" structures at the plateau edges for such large $Y$. The main problem that exists with using the Daisaka et al. formulation at face value is that the B ring inner edge does not remain sharp and rapidly diffuses even over this relatively short time scale in simulations (not shown) done using Eq. (C-1). In order to overcome such a strong viscosity in the inner $\mathrm{B}$ ring requires possibly unrealistic yields $\left(Y \gtrsim 10^{7}\right)$. If wakes are present, the viscosity may indeed be different in the plateaus compared to the lower optical depth background, but it remains to determine the nature of viscosity in the inner B ring. Overall, Fig. 14 (and Fig. 12) may simply suggest that plateaus are better sustained if the plateau viscosity is larger than the canonical kinematic viscosity case used for some reason in our models, and the inner $\mathrm{B}$ ring viscosity is more in line with expectations for the kinematic viscosity. Marouf et al. (2012) find a population of $20 \mathrm{~m}$ radius particles in the plateaus, for instance, which needs to be reconciled with UVIS data that suggests smaller local particles (Sec. 2.2.6).

\section{Discussion and Conclusions}

We have introduced a modernized evolutionary code for the modeling of micrometeoroid bombardment and ballistic transport in planetary rings that is capable of simulating both structural and compositional changes simultaneously over a broad range of spatial and temporal scales. Our code is based on the original work of Durisen and colleagues (Durisen et al., $1989 ; 1992 ; 1996)$, where they modeled the evolution of ring structure with time due 
to BT, and the pollution transport model of CE98 in which structure was held fixed and only the relative amounts of non-icy and icy constituents were allowed to evolve due to the effects of BT. A major innovation has been the parallelization of the code, which not only allows us to explore a broader range of parameter space but will eventually allow for the implementation of different ejecta distributions and the ability to treat inhomogeneous and non-axisymmetric structure. Furthermore, our code is robust enough to readily incorporate new physics (see below). These innovations are important because previous studies on ballistic transport were limited both by computational demands and a lack of key physical data to work with. Using new Cassini data, and current computing technology, more detailed in-depth investigations are possible.

Code tests. As a primary test of the code's utility, we have reproduced some of the structural results of Durisen et al. (1992, specifically their Figs. 10a, 10b and 15), who simulated a relatively sharp high- $\tau$ inner $\mathrm{B}$ ring edge and its transition into a low, constant optical depth $\mathrm{C}$ ring using constant opacity. Our results are essentially identical, with the exception of some small scale (order $\sim x$ near edges and $\sim x^{2}$ away from edges) differences in the highest yield cases, traceable to slightly different expressions for the function which relates the radius of ejection of an ejectum to that of its possible radii of absorption (Sec. 3.1). Comparing our parallel simulations to those using a serial version of our code, we found that a speedup (using $n_{p}=8$ on a quad core CPU) of roughly an order of magnitude. The increase in speed over the original serial simulations of Durisen et al. would be significantly more than this considering the processor speed of the day.

All previous simulations assumed the ring opacity $\kappa$ to be constant. We ran the same set of simulations as above, but instead used a variable opacity model (Sec. 2.2.6). This allowed us to model $\mathrm{C}$ ring plateaus which are more massive than would be predicted by a constant opacity model. The values for the opacity were scaled to the average values gleaned by CE98 for the $\mathrm{C}$ ring and inner $\mathrm{B}$ ring from occultation-variance results (Showalter and Nicholson, 1990). Using CE98 scaling for the opacity in the $\mathrm{C}$ and inner B ring leads to a surface mass density outside of the plateaus that is about five times less than in the models of Durisen et al. (1992) but leads to much more mass in the plateaus (Sec. 3.1, Fig. 5). The greater contrast in $\sigma$ between the two ring regions causes considerable differences in the amount of darkening that occurs over the same time scale for a given $Y$, with variable $\kappa$ producing a much more polluted $\mathrm{C}$ ring relative to the inner $\mathrm{B}$ ring. The variable $\kappa$ also had a considerable effect on the undulations that are seen in the inner B ring for the highest yield case $\left(Y=2 \times 10^{6}\right)$, with their growth suppressed relative to the the constant $\kappa$ case. In both opacity models, a ramp forms at the base of the inner B ring, although it is not linear in its early stages.

Long evolutions of the B ring edge and formation of the ramp. In order to demonstrate the ability of BT to maintain a steady-state inner B ring edge over much longer times than could be modeled by Durisen et al. (1992), we have run an $800 \mathrm{t}_{G}$ case with a yield of $Y=1.5 \times 10^{6}$ $\left(\sim 7 \times 10^{6}\right.$ years $)$ for a constant opacity. For the chosen set of parameters, we indeed find that the inner B ring remains sharp and relatively unchanged over the simulation timescale. We also find that the undulations in the inner B ring are approaching a steady state. The ramp region continues to develop as brighter material spreads inward over time, with the overall slope of the ramp approaching rough agreement with that seen in the observations. 
However, a hump in the ramp grows as well, in disagreement with observed structure, and its presence may contribute to the structure that appears at radii inwards of the hump's radial location. We have run a complementary case using the same parameters for the variable opacity. We find that the inner B ring edge is spreading indicating that a slightly larger $Y$ is needed in order to maintain it. The main differences, which may be due to the spreading edge, is that the inner $\mathrm{B}$ ring undulations are muted, while the $\mathrm{C}$ ring undulations appear to be longer in wavelength than in the constant opacity case.

The reason for the formation of the hump is not entirely clear, but we suspect that it is a product of the growth of BTI-like waves at an intermediate lengthscale. Higher values of $n$ lead to "notches" rather than humps in the edge that are somewhat similar to the notch seen in the observational data (see Fig. 9, dotted curve). This is because a higher value of $n$ places more ejecta in lower $x$-values which reduces the contribution to ramp production. Another effect of higher $n$ is for the B ring inner edge to round off more to larger radii, even while the slope of the edge near its base remains sharp and similar to its initial value. This is because for steeper sloped ejecta distributions, more material is concentrated at smaller $x$ which weakens the ability of BT to counter viscosity at high $\tau$. For lower $n$, while the edge remains sharp, no hump forms in the ramp region and the ramp appears quite linear (Sec. 3.2, Fig. 9) in agreement with observations. The wide difference in the effect of the variation of $n$ strongly suggests that not just the particle size distribution, but also particle properties such as hardness may vary significantly over the rings. In particular, the B ring inner edge seems to exhibit features that suggest that multiple ejecta distributions with different $\tau, n-$ and $x$-values are in play, as well as prograde and retrograde symmetries.

$C$ ring plateaus. We have run a series of models that focus on simulating how BT can maintain the observed plateau structure within Saturn's outer C ring, studying the effects of varying the yield $Y$, the strength of the kinematic viscosity $f_{\nu}$, the possible role of gravitational wakes, and the lower bound of the ejecta velocity distribution $x_{b}$. For our fiducial model parameter choices, the sharpening effect of BT for relatively small yields $\left(10^{4}\right.$, but less so for $Y=10^{5}$ ) is insufficient to overcome the effects of viscosity. Outer edges of plateaus, which should sharpen due to BT alone for a prograde ejecta distribution, smear out as quickly as inner edges. For the highest yield case $\left(Y=10^{6}\right)$ BT effects more than overcame viscosity in the $\mathrm{C}$ ring plateaus, leading to oversharpening of the plateau outer edges; however, yields $\gtrsim 10^{6}$ are required to maintain the sharpness of the inner $\mathrm{B}$ ring edge. Thus BT effects are sensitive to parameters, which may vary from place to place. The effects of BT on composition globally lead to a broad transition in $f_{\mathrm{e}}$ across the inner $\mathrm{B}$ ring edge and $\mathrm{C}$ ring, consistent with what is observed and previous models (CE98). It is most gradual for the lowest yield, which represents the longest "real" time evolution.

We also varied $f_{\nu}$ for yields of $10^{4}-10^{6}$ (while holding $x_{b}$ constant) to demonstrate the role of the kinematic viscosity in maintaining ring structure. We find that suitable combinations of $f_{\nu}$ and $Y$ can be found between $Y=10^{4}-10^{6}$, with larger $Y$ requiring larger $f_{\nu}$, that produce the best fits to the shapes of $\mathrm{C}$ ring plateaus. Agreement is not exact, but there is strong evidence for a combined dependence (through the parameter $Y / f_{\nu}$ ), as also found by Durisen et al. (1992). We have also noted that the $\mathrm{C}$ ring plateaus marginally satisfy the condition for wake formation, calling for a different viscosity model. Using the highest yield case, where large increases in particle size $\bar{r}$ are needed for a purely kinematic viscosity 
model to prevent BT from oversharpening structural features, the wake model for $\nu$ inhibits oversharpening much more effectively without having to resort to very large values of the average particle size $\bar{r}$. However, applying such a model to the inner B ring edge then requires possibly unrealistic ejecta yields $\left(Y \gtrsim 10^{7}\right)$ to maintain a sharp edge against viscous spreading.

The lower bound of the ejecta distribution defines the minimum throw distance of material resulting from an impact. The lower the ejecta velocity, the less will be the difference in the specific angular momentum between where the material was ejected and where it lands. This leads to smaller radial drift, even if the amount of material being deposited for a given $Y$ is the same. This has two effects on the plateau edges. The first is that the location of the edge is markedly different for different choices of the lower bound of the ejecta velocity distribution (less overall drift for lower $x_{b}$ ) for the same evolution time scale (Sec. 3.3, Fig. 13). Meanwhile, for all values of $x_{b}$, the slope of the outer edge remains roughly the same. Second, lower $x_{b}$ has a tendency to soften the secondary structure within the plateaus for cases in which $\nu$ is weak compared to BT, as well as the "spikiness" of the outer edges. Though lower $x_{b}$ leads to less dramatic structural changes, we find that the lower $x_{b}$ also produces more contrast in composition, because the direct term dominates the advective term, and pollution transport is much more localized.

Sensitivity to particle properties. It seems clear from our preliminary simulations that a better understanding of local particle properties is needed (i.e., $\bar{r}, r_{1}, r_{2}, r_{p}, n, x_{b}, x_{t}$, and the particle's bulk density) in order to better constrain the viscosity, yield and ejecta distribution. If the particle size distribution is different in the plateaus compared to the low $-\tau$ regions outside the plateaus, or in the ramp or inner B ring, then a viscosity that only depends on $\tau$ (or $\sigma$ ) and a constant yield is probably insufficient to describe BT over the large spatial regions we are modeling. A lower yield, as would be expected for hard targets, can match the structure of the plateaus if the viscosity is also systematically lower compared to our fiducial choice $\left(f_{\nu}=1\right)$. These values may very well need to be significantly different in the inner B ring, where Durisen et al. (1992) found that much larger yields were needed to maintain the $\mathrm{B}$ ring inner edge for a kinematic viscosity. A wake viscosity is even more problematic. Finally, not only is the ejecta distribution likely more complicated than we assume here, it is also likely variable across the rings.

Pollution of a more massive $B$ ring: We presented a toy model for the compositional evolution within the $\mathrm{B}$ ring, addressing the idea that the $\mathrm{B}$ ring may be more massive than previously thought, which would have important implications for the rings' age. We compared two massive models suggested by other workers (Robbins et al., 2008; Esposito, 2008) to a standard "Mimas" mass model in order to demonstrate both quantitative and qualitative differences in darkening over time that would occur in the B ring core, as well as the overall contrast between the $\mathrm{B}$ ring and the $\mathrm{C}$ ring. Our simulations (Fig. 10, Sec. 3.2.3) only span $100 \mathrm{t}_{G}\left(\sim 5 \times 10^{6}\right.$ years $)$, but if $\dot{\sigma}_{\infty}$ remained constant since the LHB and we extrapolate the results to cover this length of time, then the amount of darkening in the $\mathrm{B}$ ring core would be a fraction of a percent for the high end mass model, which is consistent with the work of CE98. However, the rest of the rings, especially the $\mathrm{C}$ ring and Cassini division would be far more polluted than the observations indicate. In all likelihood, the micrometeoroid flux was even higher early on, which would only serve to heighten the 
compositional differences between low and high $\tau$ regions.

\section{Future Work}

The simulations we present here, along with the work of Latter et al. (2012; 2014a,b) strongly suggest that BT (and BTI) is actively at work within the rings. In particular, the formation of ramps and the sharpening of edges can be attributed to the effects of micrometeoroid impacts and ballistic transport of impact ejecta. Our simulations clearly imply that there are still missing physics that will need to be implemented into the code. A challenge for the future will be to constrain the microphysics within the rings in order to understand how all the various effects of BT can be present at the same time. For example, how can we reconcile the form of the $\mathrm{C}$ ring plateaus, ramp and the $\mathrm{B}$ ring inner edge relative to each other? The sensitivity of our results to the variation of $\kappa, \nu, Y$ and the form of the ejecta distribution indicate that simple models in which these properties are considered to be the same across the rings are inadequate, and will need to be studied in more detail. Below, we summarize improvements that will be applied in future work.

Isotropic and Retrograde Distributions. Disruptive collisions tend to fragment the target ring particle into many pieces, leading to ejecta distributions that differ from the nondisruptive (cratering) case in terms of yield, velocities and directionality. The "yield" consists of most (or even all) of the target particle, with most of the mass located in the largest fragments (e.g., Nakamura and Fujiwara 1991) which move at much smaller velocities relative to those associated with cratering impacts. Lower relative velocities imply that the throw distances of most material in a disruptive impact are smaller, and their ejecta may mostly move in the direction of the projectile (retrograde relative to Keplerian, Paolicchi et al., 1989). Thus a considerable fraction of disruptive collisions could result in an ejecta distribution in which most of the mass may land at radii inwards of their ejection point. Projectiles which only marginally disrupt a ring particle may only result in an isotropic velocity distribution (Nakamura and Fujiwara 1991), but even this would be less prograde than the prograde distribution we assume for cratering ejecta in this paper.

Retrograde or isotropic ejecta may play a significant role in evolving local ring structure. We have already indicated that difference between the effects of prograde and retrograde velocity distributions may be the most important in the $\mathrm{C}$ ring plateaus. The characteristic outward-increasing slope in $\tau$ seen in all the plateaus outside $87500 \mathrm{~km}$ (Fig. 1) is a well known signature of BT due to prograde ejecta. Retrograde (or even isotropic) ejecta would lead to the opposite (or flattened) trend, which is seen in all the plateaus inside $87500 \mathrm{~km}$. An observation suggesting that disruptive impacts may be playing a role is recent Cassini Radio Science (RSS) data that imply there are fewer $\sim 1-3 \mathrm{~cm}$-sized particles in the plateaus than outside the plateaus (Cuzzi et al., 2009). This is significant because $\sim 1 \mathrm{~cm}$ particles may be the size most easily disrupted by meteoroid bombardment, if the peak in the micrometeoroid flux mass distribution indeed occurs near a radius of $\sim 100 \mu \mathrm{m}$. For instance, one can crudely estimate the ratio of the mass of the largest fragment to the original target mass $f_{b} \sim \mu Q_{*} / v_{\text {im }}^{2}$ (e.g., Melosh, 1989) where $\mu \sim 10^{6}$ is the corresponding target/impactor mass ratio, and $Q_{*} \sim 10^{6} \mathrm{ergs} \mathrm{g}^{-1}$ (e.g., Stewart and Leinhardt, 2009) is the 
fragmentation energy per unit mass. Including gravitational focusing, the impact velocity $v_{\mathrm{im}} \sim 30 \mathrm{~km} \mathrm{~s}^{-1}$ in the $\mathrm{C}$ ring, giving $f_{b} \sim 0.1$, well within the regime of catastrophic breakup. Whether or not this suggests that disruptive impacts occur more prominently in the plateaus is something we will explore.

Recent work that uses observations by New Horizons to estimate the flux of EKB dust at Saturn suggests that this may be an equally important source of extrinsic material (Poppe et al., 2010, Poppe and Horányi, 2012). Furthermore, though the mass peak for this population (a few microns) is likely too small to lead to disruptive impacts, these authors' models indicate that the ejecta distributions produced by EKB dust grain impacts are likely to be more isotropic (Poppe, priv. comm.), because their inflow distribution to the rings is more isotropic. Moreover, this population is associated with a large gravitational focusing factor (CD90).

Ring opacity and particle properties. The $\sim 100$ spiral density waves analyzed by Cassini UVIS and VIMS (e.g., Colwell et al., 2007; 2009; Hedman et al., 2007; Nicholson et al., 2008) have greatly improved our knowledge of key ring dynamical properties such as the optical depth and mass surface density, leaving the surface mass density unconstrained only in the dense B ring core. Other workers find that, even on smaller scales than the core region, the rings are composed of virtually opaque clumps separated by nearly transparent gaps. This structure has been attributed to self-gravity wakes, which numerical simulations have shown to form spontaneously as a result of the combined effects of self-gravity and collisional damping of particles (e.g., Salo 1995; Daisaka and Ida 1999; Ohtsuki and Emori 2000; Lewis and Stewart, 2009; Robbins et al., 2010). Nicholson et al. (2008) and Colwell et al. (2008) have exploited this expectation to separate the large and unconstrained $\tau$ within dense wakes from the $\tau$ and fractional area of the nearly transparent gaps. These workers find that, for (VIMS) occultations spanning both the $\mathrm{A}$ and $\mathrm{B}$ rings $\left(11^{\circ}<B<51^{\circ}\right)$, gap optical depths are relatively constant $(0.15<\tau<0.3)$ wherever wakes exist.

Analysis of these extensive observations (which also gives the typical pitch angle, height and spacing to width ratios of the dense wake clumps, e.g., Colwell et al., 2006; 2007) allows one to determine a local autocorrelation length scale which characterizes the physical dimensions of contiguous physical material (clumps) blocking the starlight. This scale varies depending on the viewing geometry, because the clumps are not spherical, thus complicating the search for an "effective largest particle size" that characterizes the ring opacity $\kappa$. By combining results from multiple Cassini UVIS stellar occultations that encompass a broad range of viewing geometries, it may be possible to constrain the absolute physical dimensions of the self-gravity wakes (Colwell, priv. comm.), which can then be applied to our opacity model in the BT code.

Better understanding of viscosity. According to more recent dynamical simulations (Stewart et al., 2007; Robbins et al., 2010), gravity wakes in the A ring behave quite differently than they do in the B ring. While clump lifetimes are much shorter in the A ring relative to the $\mathrm{B}$ ring, clumps extend further in the radial direction when stretched, and the angle of a wake relative to the azimuthal direction varies more with time as it evolves (Colwell et al., 2009). These differences will clearly affect the viscosity, but there is still no clear indication of how this happens or how the magnitude of the viscosity varies radially at different points in the rings. Some $N$-body simulations indicate the possibility of order 
of magnitude changes between $120000 \mathrm{~km}$ to $100000 \mathrm{~km}$ for a constant $\sigma$ (Stewart, priv. comm.). In order to apply these updated models with confidence to our BT code, we will need a clear idea of the relationship between $\nu, \sigma$ and $\tau$ over a broad range of surface density values. This is important because the simple model for wakes we have applied in this work (Daisaka et al., 2001; Figures C-1 and 14) predicts a viscosity in the inner B ring that is up to $\sim 2$ orders of magnitude greater than what is predicted from a purely kinematic viscosity, just how much greater depends on the actual $\tau$ which is a function of elevation angle, and also wake orientation if present. In any case, such a large $\nu$ would make it very difficult for BT to maintain the inner B ring edge in steady state. Work in this area is ongoing and improvements will be applied to future work as they become available. Finally, other models and parameterizations for the viscosity exist (e.g., see Schmidt et al., 2009) which we intend to explore in our models going forward.

Code modifications. Our BT code currently uses a rezoning scheme to maintain a relatively uniform width for the Lagrangian cells over the course of a simulation. If left unchecked, bins can become so narrow as to lead to unrealistic structure, or, worse, bin edges can even overlap. The process of rezoning as currently done in the BT code avoids these problems but requires some averaging, which has the effect of smoothing quantities and thus artificially affecting structure. This will likely become much more of an issue for very long simulations that may span thousands of $\mathrm{t}_{G}$. The rezoning algorithm will be revisited with the idea of developing a method for handling the merging and splitting of bins that does not lead to the systematic smoothing of structure. Since we will be interested in modeling local (finer-scale) structure as well, we feel this is a prudent endeavor.

From our results here, it would seem that the ring particle size distribution and other particle properties may vary considerably over the rings. Previous work avoided this complication because it introduced one or more parameters in the already computationally expensive calculation of the losses and gains. However, with the parallelization of the code, the ability to expand the integration of the $\Gamma$ 's and $\Lambda$ 's is no longer a roadblock to further progress. In future papers, we expect to include more treatment of variable particle properties as well expanding the number of points used in the integration over the velocity distribution $x$, which will also be useful in local, finer-scale simulations, as well as expanding the number of points used in our integrations over the azimuthal angle $\phi$.

Longer evolutions with improved physical models continue to provide encouragement that $\mathrm{BT}$ is an important process driving ring structure. The differences in how structure and composition behave under BT, and the localized versus more global transport of pollutants may be something we can distinguish from the observations. In forthcoming papers, we will be modeling both specific features as well as large scale structure based on the observed optical depths from Cassini UVIS, while the ratios of Cassini ISS color images can provide a measure of the differences in composition across these features. Ballistic transport modeling along with a combination of radiative transfer modeling in the spirit of what was done in CE98, combined with the very extensive data set provided by Cassini, might then provide much tighter constraints on how pollutants are transported within the rings. It ultimately may help to answer two long-standing questions: how old the rings really are and what they are made of. 


\section{ACKNOWLEDGEMENTS}

The authors warmly thank Henrik Latter and Jürgen Schmidt for their thoughtful and thorough reviews of this work which has led to great improvement in its exposition. We thank Josh Colwell and Glen Stewart for useful discussions. This work was supported by a grant from NASA's Cassini Data Analysis Program (PRE), and a Cassini IDS grant to JNC.

\section{Appendix A: The ejecta yield function}

We utilize a radiative transfer analog, which assumes plane-parallel symmetry and treats incoming meteoroids and their ejecta as "photons", to compute the ejecta distribution function $\mathscr{Y}$ which has been parameterized from the numerical results of CD90 in the form:

$$
\mathscr{Y}(\alpha, \beta ; R, \tau)=\frac{Y}{10^{4}} g(R, \tau) \frac{\alpha}{\alpha_{\mathrm{p}}} e^{-\left(\alpha / \alpha_{\mathrm{p}}\right)^{S} / S} \frac{\cos (\alpha / 2)}{\cos \left(\alpha_{\mathrm{p}} / 2\right)}(\cos \beta)^{m} .
$$

The ejecta distribution function is most conveniently defined in terms of the cone and clock angles $(\alpha, \beta)$ of the emergent ejecta measured from the ring particle velocity vector and are related to the zenith and azimuth angles $(\theta, \phi)$ of the emergent ejecta through the relations $\cos \theta=\sin \alpha \cos \beta$ and $\tan \phi=-\sin \beta \tan \alpha$, respectively (see Figure A-1). The function $g(R, t)$, and the parameters $\alpha_{\mathrm{p}}, m$ and $S$ are themselves functions of $\tau, \alpha$ and/or $R$ (Equations [41-46], CD90). The function $g(R, t)$ is used to calculate the probability that incoming micrometeoroids, and/or subsequent incoming or outgoing ejecta, will impact a ring particle as they pass through the rings. That is, the ejecta distribution $\mathscr{Y}$ is direction-dependent and describes the total rate of impact ejecta emission or absorption per unit area and time for a given local ring region of surface mass density $\sigma$ and optical depth $\tau$. The distribution function above assumes that impacts are cratering and non-disruptive (see Sec. 2.2.8). Figure A-2 shows an actual calculation of $\mathscr{Y}$ as a function of $\alpha$ and $\beta$ for optical depths of $\tau=1$ and $\tau=0.1$. In these plots, the prograde bias is clearly illustrated by the maxima in the distributions occurring for $\alpha<1.5$ radians. The parameterized fit in Eq. (A-1) matches the actual results quite well (see Fig. 13, CD90).

\section{Appendix B: Secondary effects to radial drift}

In this appendix, we discuss the secondary contributions to radial drift in more detail which are due to direct mass and angular momentum deposition rates. If one considers the mass deposition rate alone ("mass loading", second term on the RHS of Eq. [14]), the timescale for a ring to be hit by its equivalent mass is given by $t_{\text {masl }}=\sigma / \dot{\sigma}_{\mathrm{im}}=Y t_{\mathrm{G}}(c f$. Sec. 2.2.1). A comparable drift time from some radial location $R$ to the central body due to this term would be $\Delta R /\left|v_{\mathrm{R}}^{\text {masl }}\right|=(\Delta R / 2 R) t_{\text {masl }}$. For typical ring parameters, radial drifts due to the mass loading effect alone would cause the $\mathrm{C}$ ring and most of the $\mathrm{B}$ ring to be lost to Saturn faster than it would take for the accumulation of enough extrinsic material to equal the original $\sigma$ (Ip, 1983; 1984; Durisen et al., 1996). 
The angular momentum deposition rate leads to a net negative torque on the rings, and thus an additional radial drift component $v_{\mathrm{R}}^{\text {torq }}(\mathrm{Ip}, 1984)$. The reasons for this can be twofold. The first is that in regions of moderate or low optical depth, micrometeorites can pass through the ring without being absorbed. Recall that the reason that the ejecta flux distribution of CD90 is strongly biased in the prograde direction is due in great part to the aberrations introduced by the motions of Saturn and the orbits of the ring particles. Due to these aberrations, the slant path of the incoming micrometeoroids statistically leads to more impacts on the leading face of orbiting ring particles, and these particles are more completely absorbed. A negative torque occurs because meteoroids with negative specific angular momentum are preferentially absorbed, and this would be the case even if the micrometeoroid flux were isotropic in the frame moving with Saturn (Durisen et al., 1996). This effect does not affect very high optical depth regions because then all micrometeoroids are absorbed. A second effect that can lead to a negative torque is that a significant amount of the ejecta might escape the system, carrying with them specific angular momentum. With very large yields, even a small fraction of ejecta that might vaporize, or obtain large enough ejecta velocities could possibly lead to significant changes over very long evolutionary times, but we do not consider this possibility currently in our models ${ }^{9}$.

Durisen et al. (1996) used the techniques of CD90 (e.g., see their Fig. 15) to calculate the drift velocities due to mass loading and asymmetric micrometeoroid absorption, which causes the negative torque, as functions of optical depth for a broad range of radii and optical depths. They derived simple analytical functions of $R$ and $\tau$, which we utilize in the BT code, that capture the results of their numerical calculations. The analytical fits to the drift due to collective effects are given by

$$
\begin{gathered}
v_{\mathrm{R}}^{\mathrm{torq}}(R, \tau)=-\left[1.1 \times 10^{-8}\left(R / R_{\mathrm{S}}\right)+4.0 \times 10^{-8}\right] e^{(-\tau / 0.28)^{0.74}} \mathrm{~cm} \mathrm{~s}^{-1} \\
v_{\mathrm{R}}^{\text {masl }}(R, \tau)=-\frac{1.1 \times 10^{-8}\left(R / R_{\mathrm{S}}\right)+6.0 \times 10^{-8}}{\tau}\left(1-e^{-\tau / 0.47}\right)^{0.98} \mathrm{~cm} \mathrm{~s}^{-1}
\end{gathered}
$$

In these expressions, it is implicitly assumed that the ring mass surface density is $96 \mathrm{~g} \mathrm{~cm}^{-2}$ for $\tau=1$.

\section{Appendix C: Viscosity in the presence of self-gravity wakes}

The ubiquity of gravitational wakes in Saturn's A and B rings has emphasized the importance of the rings' own self-gravity (e.g., Colwell et al., 2007; Nicholson et al., 2008; Schmidt et al., 2009; Robbins et al., 2010). The relative abundance of these "clumps and gaps" controls the observed optical depth, but the ring viscosity is controlled by the mass and dimensions of the clumps and/or large particles. Numerical simulations demonstrate that once strong wake

\footnotetext{
${ }^{9}$ This does not violate our "thin disk" assumption in Sec. 2.2.2. Even if some ejecta obtain a relatively large $v_{\mathrm{ej}}$, the overwhelming bulk of impact ejecta will only achieve velocities in the $\sim 1-100 \mathrm{~m} \mathrm{~s}^{-1}$ range.
} 
structures form, the effective viscosity is dominated by gravitational torques due to the wake structure, because gravitational scattering by wakes increases relative random velocities, which enhances the local component of the viscosity (Salo, 1995; Daisaka and Ida, 1999; Schmidt et al.,. 2009).

In the absence of wakes, the kinematic viscosity (Eq. [24]) would dominate. On the other hand, introducing self-gravity allows the possibility for transient wake formation, as demonstrated in local $N$-body simulations (e.g., Salo, 1995; Daisaka and Ida, 1999; Ohtsuki and Emori, 2000), if an increase in the local mass surface density sufficiently decreases the Toomre parameter such that $Q=\Omega c / 3.36 G \sigma \lesssim 2$. One model which includes this physics is that of Daisaka et al. (2001), who derived an analytical expression for the viscosity in the presence of wakes that can be readily utilized in our code:

$$
\nu(R, \sigma)=26 \frac{G^{2} r_{h, 0}^{* 5}}{\Omega_{0}^{3}} \sigma^{2}\left(\frac{R}{\mathrm{R}_{S}}\right)^{19 / 2},
$$

where $r_{h}^{*}=(R / 2 r)(2 m / 3 M)^{1 / 3}$ is the scaled, mutual Hill radius of ring particles of size $r$, and thus the viscosity depends on the particle internal density. The numerical factor in Eq. (C-1) is a fit to their $N$-body simulations, and quantities with subscript 0 are evaluated at $R_{S}$.

In terms of optical depth, the condition for wake formation (Ohtsuki and Emori, 2000) can be expressed as

$$
\tau \gtrsim 0.45\left(\frac{\rho_{0}}{\rho}\right)^{1 / 2}\left(\frac{1.45 \mathrm{R}_{S}}{R}\right)^{3 / 2}
$$

where $\rho_{0}=0.5 \mathrm{~g} \mathrm{~cm}^{-3}$. This expression assumes spherical particles of a single size, and that the velocity dispersion for Saturn's rings is likely determined by the escape velocity from the largest particles (Salo, 1995; Daisaka and Ida, 1999; Ohtsuki, 1999). Equation (C-2) suggests that the gravitational instability can marginally be satisfied in the $\mathrm{C}$ ring plateaus, especially if ring particles are not porous. This is better illustrated in Figure C-1 where we plot the viscosities associated with the two models that we use for the observed optical depth. Here we have used the optical depth from the Cassini UVIS $\alpha$-Arae occultation (elevation angle $B=54.4^{\circ}$, Colwell et al., 2009), and assumed a variable opacity (Eq. [22]) with the values in the $\mathrm{C}$ ring and inner $\mathrm{B}$ ring consistent with $\mathrm{CE} 98$. In the $\mathrm{C}$ ring, the viscosity in the low- $\tau$ regions is dominated by collisions alone, whereas in the highest- $\tau$ plateaus, as well as the inner $\mathrm{B}$ ring, the viscosity could be dominated by wakes.

If the viscosity in the inner $\mathrm{B}$ ring is up to two orders of magnitude larger than what collisions alone would predict (as we would get using the Daisaka et al. formulation, see Fig. C-1), the BT mechanism would require unrealistic yields in order to maintain a sharp inner edge. The actual normal optical depth in the $\mathrm{B}$ ring outside of wakes depends on elevation angle (while in the $\mathrm{C}$ ring $\tau$ is less sensitive, Colwell et al., [2009]). However, wake models predict that the observed $\tau$ has a strong dependence on the orientation of wakes relative to the observational geometry and elevation angle, invalidating the classical scaling with elevation angle $B$ (Cuzzi et al., 2009) for at least the lower elevation occultations. 
Nevertheless, even $\tau \sim 1$ produces a rather large variation in the wake viscosity relative to the highest plateaus due to the $\sigma^{2}$ dependence. Thus the circumstances under which the viscosity in Eq. (C-1) is applicable is less straightforward to determine (see discussion, Sec. $5)$. Furthermore, the possible wake effect is magnified by our expression for $\kappa(\tau)$ (Eq. [22]), which was motivated by the plateaus and may not hold in the B ring.

\section{Appendix D: Numerical methods}

Stepping in time: In the BT code, the ring is divided into $N$ 'ringlets', or annuli with bin centers $R_{j}$. The annuli are treated as Lagrangian cells whose $N+1$ edges $b_{j}\left(b_{j}<R_{j}<b_{j+1}\right)$ drift at velocities $v_{\mathrm{R}}$ due to a combination of the ballistic transport mechanism, ring viscosity, mass loading, gain or loss of ejecta momentum, and/or torques that arise as a result of the asymmetric absorption of meteoroids (Durisen et al., 1996). The main quantities of interest: the surface density $\sigma$, optical depth $\tau$, opacity $\kappa$, viscosity $\nu$, and fractional mass of non-icy, extrinsic pollutant $f_{e}$, are evaluated at bin centers, which lie midway between the moving bin edges. Given these quantities at bin center locations $R_{j}^{n}$ at time $t^{n}$, we advance the simulation

$$
t^{n+1}=t^{n}+\Delta t^{n}
$$

using a variable timestep $\Delta t(t)$. We describe the execution of our code below.

1. We implement a rezoning scheme (Durisen et al., 1992) that searches over the radial grid to find annuli that have become narrower or wider than pre-assigned limits. If the condition is satisfied, the narrowest bin is merged with its smallest neighbor, while the largest bin is split in two. The physical quantities of a merged or split bin are adjusted accordingly. Typical minimum and maximum limits are 2/5 and 5/2 the mean initial bin size, respectively, where the mean bin size is the radial extent of the grid divided by $N$.

2. The viscosities $\nu_{j}^{n}$ are updated from the last timestep $t^{n}$ using our chosen viscosity law (see Sec. 2.2.7 and Appendix B).

3. The appropriate timestep size $\Delta t^{n}$ for the current iteration is determined from the stability criteria (see below).

4. The losses $\Lambda^{n}$ and gains $\Gamma^{n}$ are determined at every $R_{j}^{n}$. Our method of integration (described below) uses a lookup table of current values of the fractional mass, the optical depth and other quantities that depend on these parameters. Thus, prior to integration, the relevant quantities are interpolated onto a very fine grid.

5. The drift velocity due to ballistic transport at bin center $R_{j}$ is calculated from Eq. (12) using the difference formula

$$
\left(v_{\mathrm{R}}^{\mathrm{ball}}\right)_{j}^{n}=\frac{2 \sqrt{R_{j}}}{\sigma_{j}^{n}}\left[\Gamma_{j, \mathrm{~h}}^{n}-\Lambda_{j, \mathrm{~h}}^{n}-\sqrt{R_{j}}\left(\Gamma_{j, \mathrm{~m}}^{n}-\Lambda_{j, \mathrm{~m}}^{n}\right)\right] .
$$

6. The viscous drift velocity Eq. (13) is calculated at points midway between bin centers using the differencing 


$$
\left(v_{\mathrm{R}}^{\mathrm{visc}}\right)_{j-1 / 2}^{n}=-\frac{3}{\bar{\sigma}_{j-1}^{n} \bar{R}_{j-1}^{n}} \frac{(\sigma \nu \sqrt{R})_{j}^{n}-(\sigma \nu \sqrt{R})_{j-1}^{n}}{R_{j}^{n}-R_{j-1}^{n}},
$$

where all quantities are evaluated at time $t^{n}$, and the bar represents an average of the quantity between spatial bins $j$ and $j-1$ (e.g., $\bar{R}_{j-1}=\left(R_{j}+R_{j-1}\right) / 2$ ). The viscous drift velocities are then interpolated to the true bin edges which generally do not lie midway between bin centers.

7. If mass loading and ring torques are included in the simulation, then corresponding velocities (which depend on $\tau_{j}^{n}$ ) are calculated at bin centers using Eqns. (B-1) and (B-2) from Appendix B.

8. The total changes to the ring surface mass density due to direct deposition terms (RHS of Eq. [8]), as well as direct changes to the surface mass densities of extrinsic and intrinsic materials, are calculated using

$$
\begin{gathered}
\sigma_{j, \mathrm{dir}}^{n+1}=\sigma_{j}^{n}+\Delta t^{n}\left[\left(\dot{\sigma}_{\mathrm{im}}\right)_{j}+\Gamma_{j, \mathrm{~m}}^{n}-\Lambda_{j, \mathrm{~m}}^{n}\right] \\
\left(\sigma_{\mathrm{e}}\right)_{j, \mathrm{dir}}^{n+1}=\left(f_{\mathrm{e}}\right)_{j}^{n} \sigma_{j}^{n}+\Delta t^{n}\left[\eta f_{\mathrm{ext}}\left(\dot{\sigma}_{\mathrm{im}}\right)_{j}+\Delta \dot{\sigma}_{\mathrm{e}}\right] \\
\left(\sigma_{\mathrm{i}}\right)_{j, \mathrm{dir}}^{n+1}=\left[1-\left(f_{\mathrm{e}}\right)_{j}^{n}\right] \sigma_{j}^{n}+\Delta t^{n}\left[\left(1-\eta f_{\mathrm{ext}}\right)\left(\dot{\sigma}_{\mathrm{im}}\right)_{j}+\Delta \dot{\sigma}_{\mathrm{i}}\right]
\end{gathered}
$$

where $\Delta \dot{\sigma}_{\mathrm{e}}$ and $\Delta \dot{\sigma}_{\mathrm{i}}$ represent the integrals on the RHS of Eqns. (10) and (11), respectively. The new fractional mass due only to direct terms is then calculated from $\left(f_{\mathrm{e}}\right)_{j \text {,dir }}^{n+1}=$ $\left(\sigma_{\mathrm{e}}\right)_{j, \mathrm{dir}}^{n+1} / \sigma_{j, \mathrm{dir}}^{n+1}$.

9. The "indirect" contributions to changes in $\sigma, \sigma_{\mathrm{e}}$ and $\sigma_{\mathrm{i}}$ are due to differential drift. Bin-centered velocity contributions to the drift velocity $v_{\mathrm{R}}$ are interpolated to bin edges, and then combined to evolve bin edges to their new positions at $t^{n+1}$ :

$$
b_{j}^{n+1}=b_{j}^{n}+\Delta t^{n}\left(v_{\mathrm{R}}\right)_{j}^{n} .
$$

The new bin centers are then taken to be midway between the new bin edges $R_{j}^{n+1}=$ $\left(b_{j+1}^{n+1}+b_{j}^{n+1}\right) / 2$.

10. The divergence or indirect term contribution to the surface mass density on the LHS of Eq. (8) then is calculated by modifying the direct term to account for bin compression or expansion

$$
\sigma_{j}^{n+1}=\sigma_{j, \operatorname{dir}}^{n+1} \frac{\mathcal{A}_{j}^{n}}{\mathcal{A}_{j}^{n+1}},
$$

where $\mathcal{A}_{j}^{n}$ and $\mathcal{A}_{j}^{n+1}$ are the computed areas of the annuli $j$ at times $t^{n}$ and $t^{n+1}$. The divergence term contributions to $\sigma_{\mathrm{e}}$ and $\sigma_{\mathrm{i}}$ is taken into account by the same technique.

11. The optical depths $\tau_{j}^{n+1}$ are calculated using the new values of the ring surface mass density and our chosen model for the opacity which can itself depend on the optical depth or surface density (see Sec. 2.2.6). 
Stability and time step restrictions: The BT code uses a variable timestep $\Delta t(t)$ which is determined at each step to satisfy several conditions. The Courant condition (e.g., see Press et al., 1992) that arises from Eq. (8) requires that

$$
\Delta t \lesssim \min \left\{\frac{2 w_{j}}{\left|\left(v_{\mathrm{R}}\right)_{j+1}^{n}+\left(v_{\mathrm{R}}\right)_{j}^{n}\right|}\right\},
$$

where the bin width is $w_{j}=b_{j+1}-b_{j}$ and an average is used of the total drift velocities $v_{\mathrm{R}}$, which have been defined at bin edges by step 9 above. The condition essentially demands that the surface density in the bin being evaluated is not receiving information from beyond the boundaries of the spatial regions defined by each grid cell. A second condition that we impose is the viscous Courant condition. Code stability requires that the minimum timestep be shorter than half the shortest viscous diffusion time across an annular cell, namely

$$
\Delta t \lesssim \min \left\{\frac{1}{2}\left[\frac{2 w_{j}^{2}}{3\left(\nu_{j+1}+\nu_{j}\right)}\right]\right\} .
$$

The viscous contribution to the drift velocity is present in $v_{\mathrm{R}}$, but the stability criterion in Eq. (D-7) is typically less important for simulations with viscosity which we generally consider.

A last condition that the BT code must adhere to is

$$
\Delta t \lesssim \min \left\{\frac{\sigma_{j}^{n}}{\left(\dot{\sigma}_{\mathrm{im}}\right)_{j} Y}\right\},
$$

which essentially states that the time step must be less than the local gross erosion time of the ring. This obviously applies most strongly in situations where the surface density of the ring is very low, but not zero, such as near gaps or true edges. Radial cells that are empty $(\sigma=0)$ are not considered in setting $\Delta t$. At the beginning of each time step, these three conditions are checked, and the new $\Delta t$ is taken to be the minimum of these, or a default maximum time step size which is chosen at the beginning of our simulation. The maximum $\Delta t_{\max }$ is $0.00005-0.001 t_{G}$, depending on the parameter choices for a given run.

Numerical technique for calculating $\Lambda$ 's and $\Gamma$ 's: Numerical integration of the loss and gain integrals (Sec. 2.2.5) represents the most computationally intensive piece of the BT code. The integration over $\theta$ is done using a $N_{\theta}=21$ point Simpson rule evenly spaced in $\cos \theta$, while integration over $\phi$ is done using two Gaussian quadrature points $N_{\phi}$ over the azimuthal angle $\phi=\pi / 2$ to $\pi$ (the CD90 ejecta angular distribution function possesses reflection symmetry through the planes defined by $\phi=0$ and $\phi=\pi / 2$, see Fig. A-2, and Durisen et al., 1992). Since we primarily use a power-law representation of the velocity distribution (Sec. 2.2.8), the $x$-integration is done using a trapezoidal rule with typically $N_{x}=10-20$ quadrature points evenly spaced in $\log x$.

The value of the $\theta$-integrand at a given Simpson point is determined by interpolation over a fine grid of the values for the fractional mass, optical depth, and optical depth-dependent functions. Prior to each numerical integration, we pre-interpolate a much finer mesh of points $N_{\text {mesh }} \gg N$ where the array index of the mesh is chosen to be directly proportional to radial 
position. This allows for integrands to be evaluated using a mesh value that is closest to the actual value of $R$ or $R^{\prime}$ (Durisen et al., 1989). This procedure somewhat mitigates the costs of doing the angular integrals which would require $\sim N_{\phi} \times N_{\theta} \times N$ distinct searches and interpolations otherwise. Our mesh size ranges from $N_{\text {mesh }}=(50-100) \times N$. Use of the fine mesh provides a boost to computational speed at the expense of a small loss in accuracy.

After other efforts to make the integrations of the losses and gains more optimal and efficient, significant improvements in speed are best achieved through parallelization of the $\Gamma$ and $\Lambda$ integration subroutine. This was done using the language independent communication protocol Open MPI (Message Passing Interface). Prior to reaching the losses and gains integration routine, the ring (composed of $N$ annuli) is divided into $n_{p}$ chunks, where $n_{p}$ is the number of processors ("tasks") being utilized, with each task executing its "piece' of the loss and gain integration loops. After the integration is completed, the changes in the values of each radial interval must be communicated to all processors before the global calculation can continue. That is, outside of the losses and gains routine, the code runs $n_{p}$ separate, but identical versions of the simulation until the next time step call to integrate. This represents the minimum amount of communication necessary between tasks in order to ensure the code runs like the serial version. In order to achieve a good balance of computational workload between tasks, the code dynamically adjusts how many radial bins are devoted to each task based on how much time the previous time step took over the various radial ranges. This approach to optimization is based on the assumption that the system will not change too drastically each $\Delta t$. Given that the time steps we use are relatively small, the radial ranges change only gradually, and thus the resulting computations remain quite similar.

The amount of speed up in code execution achieved through our simplest parallelization approach compared to the original BT code of Durisen and colleagues scales as the number of processors $\left(\sim n_{p}\right)$ on a single node since the number of operations involved in computing the loss and gain integrals scales as the number of radial bins $(N)$. Typically a single node (e.g., a quad core CPU) will have $n_{p}=8$ processors, which is what we have used for the bulk of the simulations for this paper. We have done some cases using a CPU with $n_{p}=12$. Thus, using the minimal communication parallelization, we achieve a speedup of a factor of $\sim 10$ compared to the serial BT code. We have developed a fully parallelized version of the BT code (maximum amount of communication), which will likely be useful when expanding the size of the radial grid (i.e., global simulations with many radial bins) and requiring multiple nodes to do so. Multiple nodes naturally introduces another layer of communication to contend with, communication between nodes in addition to tasks within a node. However, for the demonstrative simulations we present in this paper, the minimum communication BT code is more than sufficient. 


\section{References}

Araki, S., and Tremaine, S., 1986. The Dynamics of Dense Particle Disks. Icarus 65, 83-109.

Burns, J. A., Showalter, M. R., and Morfill, G. E., 1984. The Ethereal Rings of Jupiter and Saturn. In Planetary Rings, (Greenberg, R., and Brahic, A., eds.), Univ. of Arizona Press, Tucson, pp 200-272..

Charnoz, S., Dones, L., Esposito, L. W., Estrada, P. R., and Hedman, M. M., 2009. Origin and Evolution of Saturn's Ring System. In Saturn from Cassini-Huygens, (Dougherty, M. K., Esposito, L. W., and Krimigis, S. M., eds.), Springer-Verlag Press, pp. 537-576.

Clark, R. N., Cruikshank, D. P., Jaumann, R., Brown, R. H., Stephan, K., Dalle Ore, C. M., Eric, L. K., Pearson, N., Curchin, J. M., Hoefen, T. M., Buratti, B. J., Filacchione, G., Baines, K. H., And Nicholson, P. D., 2012. The Surface Composition of Iapetus: Mapping Results from Cassini VIMS. Icarus 218, 831-860..

Colwell, J. E., Esposito, L. W., and Sremčevíc, M., 2006. Self-gravity wakes in Saturn's A ring measured by stellar occultations from Cassini. Geophys. Res. Lett. 33, L07201.

Colwell, J. E., Esposito, L. W., Sremčevíc, M., Stewart, G. R., and McClintock, W. E., 2007. Self-gravity wakes and radial structure of Saturn's B ring. Icarus 190, 127-144.

Colwell, J. E., Nicholson, P. D., Tiscareno, M. S., Murray, C. D., French, R. G., and Marouf, E. A., 2009. The Structure of Saturn's Rings. In Saturn From Cassini-Huygens, (Dougherty, M. K., Esposito, L. W., and Krimigis, S. M., eds.), Springer-Verlag Press, pp. 375-412.

Colwell, J. E., Esposito, L. W., Lissauer, J. J., Jerousek, R. G., And Sremčevíc, M., 2008. Three-dimensional Structure of Saturn's Rings from Cassini UVIS Stellar Occultations. European Planetary Science Congress, Proceedings, September 2008, p. 759.

Colwell, J. E., Cooney, J. H., Esposito, L. W., And Sremčevíc, M., 2012. Particle Sizes and Small-Scale Structure in Saturns Rings from Stellar Occultation Statistics. Proceedings of the 44th DPS Meeting, abstract no. 501.05..

Cuzzi, J. N., Durisen, R. H., Burns, J. A., And Hamill, P., 1979. The Vertical Structure and Thickness of Saturn's Rings. Icarus 38, 54-68.

Cuzzi, J. N., Lissauer, J. J., Esposito, L. W., Holberg, J. B., Marouf, E. A., Tyler, G. L, And Boischot, A., 1984. Saturn's Rings: Properties and Processes. In Planetary Rings, (Greenberg, R., and Brahic, A., eds.), Univ. of Arizona Press, Tucson, pp. 73-199.

Cuzzi, J. N., And Durisen, R. H., 1990. Meteoroid Bombardment of Planetary Rings; General Formulation and Effects of Oort Cloud Projectiles. Icarus 84, 467-501.

Cuzzi, J. N., Clark, R., Filacchione, G., French, R., Johnson, R., Marouf, E., and Spilker, L., 2009. Ring particle composition and size distribution. In Saturn From CassiniHuygens, (Dougherty, M. K., Esposito, L. W., and Krimigis, S. M., eds.), SpringerVerlag Press, pp. 459-510.

Cuzzi, J. N., And Estrada, P. R., 1998. Compositional Evolution of Saturn's Rings Due to Meteoroid Bombardment. Icarus 132, 1-35.

Daisaka, H., And IDA, S., 1999. Spatial structure and coherent motion in dense planetary 
rings induced by self-gravitational instability. Earth, Plan., and Spa. 51, 1195-1213.

Daisaka, H., TANaka, H., And IdA, S., 2001. Viscosity in a dense planetary ring with selfgravitating particles. Icarus 154, 296-312.

Doyle, L. R., Dones, L., And Cuzzi, J. N., 1989. Radiative Transfer Modeling of Saturn's Outer B Ring. Icarus 80, 104-135.

Durisen, R. H., 1984A. Transport Effects Due to Particle Erosion Mechanisms. In Planetary Rings, (Greenberg, R., and Brahic, A., eds.), Univ. of Arizona Press, Tucson, pp. 416-446.

Durisen, R. H., 1984B. Particle Erosion Mechanisms and Mass Redistribution in Saturn's Rings. Adv. Space Res. 4, 13-19.

Durisen, R. H., Cramer, N. L., Murphy, B. W., Cuzzi, J. N., Mullikin, T. L., Cederbloom, S. E., 1989. Ballistic Transport in Planetary Ring Systems due to Particle Erosion Mechanisms. I. Theory, Numerical Methods, and Illustrative Examples. Icarus 80, $136-166$.

Durisen, R. H., Bode, P. W., Cuzzi, J. N., Cederbloom, S. E., and Murphy, B. W., 1992. Ballistic Transport in Planetary Ring Systems due to Particle Erosion Mechanisms. II. Theoretical Models for Saturn's A- and B-Ring Inner Edges. Icarus 100, 364-393.

Durisen, R. H., 1995. An Instability in Planetary Rings Due to Ballistic Transport. Icarus 115, 66-85.

Durisen, R. H., Bode, P. W., Dyck, S. G., Cuzzi, J. N., Dull, J. D., And White II, J. C., 1996. Ballistic Transport in Planetary Ring Systems due to Particle Erosion Mechanisms. III. Torques and Mass Loading by Meteoroid Impacts. Icarus 124, 220-236.

Elliott, J. P., And Esposito, L. W., 2011. Regolith Depth Growth on an Icy Body Orbiting Saturn and Evolution of Bidirectional Reflectance due to Surface Composition Changes. Icarus 212, 268-274.

Esposito, L. W., 1987. Structure and Evolution of Saturn's Rings. Icarus 67, 345-357.

Esposito, L. W., Colwell, J. E., Larsen, K., McClintock, W. E., Stewart, A. I. F., Tew Hallett, J., Shemansky, D. E., Ajello, J. M., Hansen, C. J., Hendrix, A. R., West, R. A., Keller, H. U., Korth, A., Pryor, W. R., Reulke, R., and Yung, Y., 2005. Ultraviolet Imaging Spectroscopy Shows an Active Saturnian System. Science 307, 1251-1255.

Esposito, L. W., 2008. History of Saturn's Rings from UVIS Observations. European Planetary Science Congress, Proceedings, September 2008, p. 757.

Estrada, P. R., And Cuzzi, J. N., 1996. Voyager Observations of the Color of Saturn's Rings. Icarus 122, 251-272.

Estrada, P. R., Cuzzi, J. N., and Showalter, M. R., 2003. Voyager Color Photometry of Saturn's Main Rings: A Correction. Icarus 166, 212-222.

Estrada, P. R., And Durisen, R. H., 2011. Modeling the Long Term Evolution of the C Ring due to Meteoroid Bombardment. Proceedings of the Rings 2011 Workshop, Ithaca, NY. 
Goldreich, P., And Tremaine, S., 1978. The Velocity Dispersion in Saturn's Rings. Icarus 34, 227-239.

Goldreich, P., and Tremaine, S., 1982. Dynamics of Planetary Rings. Ann. Rev. Astron. Astrophys. 20, 249-283.

Gomes, R., Levison, H. F., Tsiganis, K., and Morbidelli, A., 2005. Origin of the Cataclysmic Late Heavy Bombardment Period of the Terrestrial Planets. Nature 435, 466-469.

Grün, E., Zook, H. A., Fechtig, H., and Giese, R. H., 1985. Collisional Balance of the Meteoroid Complex. Icarus 62, 244-272.

Hahn, J. M. 2008. The secular evolution of a close ring-satellite system: the excitation of spiral density waves at a nearby gap edge. Astrophys. J. 680, 1569-1581.

Hartmann, W. K., 1985. Impact Experiments. 1. Ejecta Velocity Distributions and Related Results from Regolith Targets. Icarus 35, 1-26.

Hartmann, L., 2009. Accretion Processes in Star Formation, Cambridge University Press, 2nd ed., Cambridge, UK, 2009.

Hedman, M. M., Nicholson, P. D., Salo, H., Wallis, B. D., Buratti, B. J., Baines, K. H., Brown, R. H., ANd Clark, R. N., 2007. Self-gravity wake structures in Saturn's A ring revealed by Cassini VIMS. Astron. J. 133, 2624-2629.

Hedman, M. M., and Nicholson, P. D., 2013. Kronoseismology: Using Density Waves in Saturn's C Ring to Probe the Planet's Interior. Astron. J. 146, 16pp.

IP, W.-H., 1983. Collisional Interactions of Ring Particles: Ballistic Transport Process. Icarus 54, 253-262.

IP, W.-H., 1984. Ring Torque of Saturn from Interplanetary Meteoroid Impact. Icarus 60, $547-552$.

Landgraf, M., Baggaley, W. J., Grün, E., Krüger, H., And Linkert, G., 2000. Aspects of the Mass Distribution of Interstellar Dust Grains in the Solar System from In-situ Measurements. J. Geophys. Res. 105, 10343-10352.

Lange, M. A., And Ahrens, T. J., 1982. Impact Cratering in Ice and in Ice-silicate Targets. Lunar Planet. Sci. 13, 530-531.

Lange, M. A., And Ahrens, T. J., 1987. Impact Experiments in Low-temperature Ice. Icarus 69, 506-518.

Latter, H., Ogilvie, G., and Chupeau, M., 2012. The Ballistic Transport Instability in Saturn's Rings I: Formalism and Linear Theory. Mon. Not. Roy. Astron. Soc. 427, 2336-2348.

Latter, H., Ogilvie, G., and Chupeau, M., 2014a. The Ballistic Transport Instability in Saturn's Rings II: Nonlinear Wave Dynamics. Accepted to MNRAS. arXiv:1404.4245.

Latter, H., Ogilvie, G., and Chupeau, M., 2014B. The Ballistic Transport Instability in Saturn's Rings III: Numerical Simulations. Accepted to MNRAS. arXiv:1404.4247.

Lewis, M. C., And Stewart, G. R., 2009. Features around embedded moonlets in Saturn's rings: The role of self-gravity and particle size distributions. Icarus 199, 387-412.

Lissauer, J. J., And Cuzzi, J. N., 1982. Resonances in Saturn's Rings. Astron. J. 87, 1051- 
1058.

Lissauer, J. J., 1984. Ballistic Transport in Saturn's Rings: An Analytic Theory. Icarus 57, 63-71.

Lissauer, J. J., And Cuzzi, J. N., 1985. Rings and Moons - Clues to Understanding the Solar Nebula. In Protostars and Planets, (Black, D., and Matthews, M. S., eds.), Univ. of Arizona Press, Tucson.

Marouf, E. A., French, R. C., Rappaport, N., McGhee, C., Wong, K., Thompson, F., and Anabtawi, A., 2008. Structure and physical properties of Saturn's rings from Cassini radio occultations. Proceedings of the "Saturn After Cassini/Huygens" meeting, held at Imperial College, London, July 28 - August 1, 2008.

Marouf, E. A., Wong, K., French, R., and Rappaport, N., 2012. Particle Size Distribution in Saturns Ring C. Proceedings of the 44th DPS Meeting, abstract no. 501.03..

Melosh, H. J., 1989. Impact Cratering: A Geological Process. Oxford Univ. Press, New York.

Morfill, G. E., Fechtig, H., Grün, E., And Goertz, C., 1983. Some Consequences of Meteoroid Bombardment of Saturn's Rings. Icarus 55, 439-447.

Nakamura, A., And Fujiwara, A., 1991. Velocity distributions of fragments formed in a simulated collisional disruption. Icarus 92, 132-146.

Nicholson, P. D., Hedman, M. M., Clark, R. N., Showalter, M. R., Cruikshank, D. P., Cuzzi, J. N., Filacchione, G., Capaccioni, F., Cerroni, P., Hansen, G. B., Sicardy, B., Drossart, P., Brown, R. H., Buratti, B. J., Baines, K. H., and Coradini, A., 2008. A close look at Saturn's rings with Cassini VIMS. Icarus 193, 182-212.

Northrop, T. G., And Connerney, J. E. P., 1987. A Micrometeorite Erosion Model and the Age of Saturn's Rings. Icarus 70, 124-137.

Ohtsuki, K., And Emori, H., 2000. Local $N$-body simulations for the distribution and evolution of particle velocities in planetary rings. Astron. J. 119, 403-416.

Paolicchi, P., Cellino, A., Farinella, P., and Zappalá, V., 1989. A Semiempirical Model of Catastrophic Breakup Processes. Icarus 77, 187-212.

Poppe, A., James, D., Jacobsmeyer, B., And Horányi M., 2010. First results from the Venetia Burney Student Dust Counter on the New Horizons mission. Geophys. Res. Lett. 37, L11101-L11106.

Poppe, A., And Horányi, M., 2012. On the Edgeworth-Kuiper belt dust flux at Saturn. 43rd LPSC Meeting, abstract no. 1365.

Poulet, F., And Sicardy, B., 2001. Dynamical Evolution of the Prometheus-Pandora System. Mon. Not. Roy. Astron. Soc. 322, 343-355.

Press, W. H., Flannery, B. P., Teukolsky, S. A., and Vetterling, W. T., 1992. Numerical Recipes in Fortran. Cambridge University Press.

Robbins, S. J., Stewart, G. R., Lewis, M. C., Colwell, J. E., And Sremčevíc, M., 2010. Estimating the Masses of Saturns A and B Rings from High-optical Depth $N$-body Simulations and Stellar Occultations. Icarus 206, 431-445. 
SAlo, H., 1991. Numerical Simulations of Dense Collisional Systems. Icarus 90, 254-270.

SALO, H., 1995. Simulations of dense planetary rings. III. Self-gravitating identical particles. Icarus 117, 287-312.

Schmidt, J., Ohtsuki, K., Rappaport, N., Salo, H., and Spahn, F., 2009. Dynamics of Saturn's dense rings. In Saturn After Cassini-Huygens, (Dougherty, M. K, Esposito, L. W.,and Krimigis, S. M., eds.), Springer-Verlag Press, pp.413-458.

Showalter, M. R. And Nicholson, P. D., 1990. Saturn's rings through a microscope - Particle size constraints from the Voyager PPS scan. Icarus 87, 285-306.

SremČevíc, M., Krivov, A. V., Krüger, H., and Spahn, F., 2005. Impact-generated Dust Clouds Around Planetary Satellites: Model Versus Galileo Data. Plan. Space Sci. 53, 625-641.

Stewart, G. R., Robbins, S. J., and Colwell, J. E., 2007. Evidence for a primordial origin of Saturn's rings. 39th DPS meeting, abstract no. 7.06.

Stewart, S. T., and Leinhardt, Z. M., 2009. Velocity-dependent catastrophic disruption criteria for planetesimals. Astrophys. J. Lett. 691, L133-L137.

Wisdom, J., and Tremaine, S., 1988. Local simulations of planetary rings. Astron. J. 95, 925-940.

Zebker, H. A., Marouf, E. A., And Tyler, G. L., 1985. Saturn's Rings - Particle Size Distributions for Thin Layer Model. Icarus 64, 531-548. 
Figure 1: The optical depth profile from the UVIS Cassini $\alpha$-Arae occultation (Colwell et al., 2009) as a function of radius from Saturn in planetary radii. Top panel: Much of the structure in the $\mathrm{C}$ ring has long remained a mystery; however, there is evidence to suggest ballistic transport is at work, particularly with the inner B ring edge sharpness and its associated ramp, and the outer (and inner) edges of plateaus. The radial drift due to ballistic transport can lead to a pile up of material at plateau edges, which may be characterized by the sloped tops of the plateaus. The reversal of the slopes of plateau peaks about the Maxwell ringlet at $\sim 1.45 \mathrm{R}_{S}$ may be indicative of impacts that change in nature to fragmenting from cratering across the region. Bottom panel: The Cassini division and inner $\mathrm{A}$ ring displays some of the same properties as the $\mathrm{C} /$ inner $\mathrm{B}$ ring. A similar ramp connects to a sharp inner edge. Even though the radial scales in the plots are different, the ramp width is roughly the same in both cases.

Figure 2: Reproduction of Fig. 10a from Durisen et al. (1992) where $Y=3 \times 10^{5}$ and $x_{b}=2 \times 10^{-4}$. The run spans $99 \mathrm{t}_{G}$ with $\tau(R)$ plotted at selected times (black curves). Also plotted are the associated fractional masses of pollutant (red curves) for these structural runs using $f_{\text {ext }}=0.5$ and $\eta=0.12$. Because the opacity is assumed to be constant, there is not much contrast between the $\mathrm{C}$ ring and inner $\mathrm{B}$ ring in composition at longer times. Our structural results are identical to Durisen et al. (1992); the edge is sharpening and a ramp is emerging (Sec. 3.1).

Figure 3: Reproduction of Fig. 10b from Durisen et al. (1992) which details the ramp and $\mathrm{B}-\mathrm{C}$ boundary region further inwards into the $\mathrm{C}$ ring at different times (black curves). The compositional evolution is shown in red. Although there is more contrast in composition for the longest time here, the variation is due to brighter material spilling over from the inner $\mathrm{B}$ ring edge into the $\mathrm{C}$ ring. The net contrast across the $1.49-1.54 \mathrm{R}_{S}$ region remains roughly constant. The undulations seen may come as a result of the BTI.

Figure 4: Reproduction of Fig. 15 from Durisen et al. (1992) which is a compilation of three different simulations for $Y=3 \times 10^{5}$ (dashed curve), $10^{6}$ (dotted curve) and $2 \times 10^{6}$ (solid curve) over a simulation time of $104 \mathrm{t}_{G}$. The models are plotted against the Voyager PPS imaging optical depth. The larger yield models use a smaller $x_{b}=10^{-4}$ and have higher resolution. Our structural results are essentially identical to Durisen et al. (1992). The compositional models are shown in red. In this case, the strongest compositional gradient is seen in the highest yield case because of the much shorter absolute timescale involved (also discussed by CE98).

Figure 5: A complementary suite of simulations to Fig. 4 in which we have employed the variable opacity model (Eq. [22]). This model gives a slightly larger mass in the inner B ring, and more mass in the plateaus, but five times less mass, overall, in the $\mathrm{C}$ ring compared to the constant opacity model. This is quite evident in the compositional evolution (red curves) where now much larger absolute contrasts in fractional mass are seen compared to the constant opacity model (the scale is the same as Fig. 4). The major structural difference after $104 \mathrm{t}_{G}$ is in the inner $\mathrm{B}$ ring where, in particular, the undulatory structure for the $2 \times 10^{6}$ case is quite suppressed. 
Figure 6: A plot of the time rate of change of optical depth as a function of ring radius for the $Y=2 \times 10^{6}$ model in Fig. 4. These plots break down the individual contributions to structural change from purely BT effects such as mass transfer and advection (of BT only) in the top panel, and the effect of viscosity on the total in the bottom panel. The first undulation (the steady-state edge) is visible around $R \approx 1.525 \mathrm{R}_{S}$. Outside of this location, the growth of quasi-steady-state undulations in the inner $\mathrm{B}$ ring is due to a lack of cancellation between BT and viscosity (the net effect is plotted in red). This actually leads to uninhibited growth in amplitude over much longer timescales. On the other hand, the strong variations inside the inner B ring edge, which are due almost entirely to BT, tend to vary in location and thus the effects tend to average out over time so that similar large amplitude growth of structure is not seen. However, these variations do lead to structure at later times (e.g., see Fig. 8).

Figure 7: A plot of the time rate of change of optical depth as a function of ring radius for the $Y=2 \times 10^{6}$ model in Fig. 5 which uses the variable opacity model. In this case, the lack of undulations in the inner B ring is explained by both the smaller amplitude of the effects of BT and viscosity, and a higher level of cancellation between them (red curve). A combination of slightly more mass in the inner $\mathrm{B}$ ring, as well as much less mass in the $\mathrm{C}$ ring, and the variable $\kappa$ combine to prevent large amplitude growth.

Figure 8: A long term simulation of the structural evolution of the inner B ring edge for $Y=1.5 \times 10^{6}, x_{b}=10^{-4}$ and $f_{\nu}=1$ for a constant opacity model. The total simulation time is $800 \mathrm{t}_{G}$ which corresponds to an absolute timescale of $\sim 7 \times 10^{6}$ years for this set of parameters. The simulation clearly shows how BT can maintain a sharp edge for long periods. A ramp forms, but develops a great deal of structure which is not present in the observations (dot-dashed curve). Also shown is the $800 \mathrm{t}_{G}$ profile (red curve) for the variable opacity model. See Sec. 3.2 for more details.

Figure 9: Suite of models for $Y=3 \times 10^{5}, x_{b}=10^{-4}$ and $f_{\nu}=0.125$ that employ different steepness in the slope of the ejecta velocity distribution (Eq. [25], $n=3$ is our adopted fiducial value) plotted against the $\alpha$-Arae occultation $\tau$ (dotted curve). The variation leads to two effects. First, lower $n$ sharpens the inner edge much more effectively than our fiducial value (for the same parameter choices) while seemingly producing a large, featureless ramp (although with less steepness). Second, larger $n$ produce "notch" like features in the edge not unlike that seen in the real optical depth profile.

Figure 10: Two hypothetical massive B ring models in which both structure (black curves) and pollution transport (red curves) evolve over time $\left(100 \mathrm{t}_{G}\right)$. The variable opacity model is used along with a modified kinematic viscosity in which the magnitude of the viscosity at the inner and outer B ring edges is chosen to maintain the edges close to their initial sharpness. The dashed curves correspond to a model in which the B ring core (highest optical regions between $\sim 1.65-1.85 \mathrm{R}_{S}$ ) has $\sigma \sim 400 \mathrm{~g} \mathrm{~cm}^{-2}$; the dotted curves have a core $\sigma=1000$ $\mathrm{g} \mathrm{cm}^{-2}$. A significant difference in the core composition is seen between the two models. The traditional "Mimas" mass model is also shown (solid curves) for comparison. The initial condition for the Mimas mass model only is given by the blue curve. For more details, see 
Sec. 3.2.

Figure 11: Compilation of combined structural and compositional models with $Y=10^{4}$ (solid curves), $10^{5}$ (dotted curves) and $10^{6}$ (dashed curves) using the $\alpha$-Arae occultation $\tau$ as the initial condition (blue curve). The simulations are run over $2 \mathrm{t}_{G}$ in the active radial range of $1.4-1.53 \mathrm{R}_{S}$ and assume $x_{b}=10^{-4}$, a Wisdom and Tremaine kinematic viscosity with $f_{\nu}=1$ and a variable opacity model. See Sec. 3.3 for explanation.

Figure 12: Evolution of plateau features centered at $1.465 \mathrm{R}_{S}$ using a constant $Y / f_{\nu}=10^{5}$ over $2 \mathrm{t}_{G}$ that demonstrates the role of the kinematic viscosity in maitaining ring structure. For the yields of $10^{4}$ (black curve) and $10^{6}$ (blue curve), the dominant particle size $\bar{r}$ is defined to be 20.4 and $204 \mathrm{~cm}$, respectively. Despite the large variation in timescale associated with the different yields, the profiles look similar due to the degree of scaling that exists between $f_{\nu}$ and $Y$ (see text). Also due to the different timescales, there has been some radial registration of the profiles to line them up for easier comparison.

Figure 13: Both the structural and compositional evolution of the same plateaus as Fig. 12 for $Y=10^{5}$ and $f_{\nu}=0.3\left(x_{b}=10^{-4}\right.$, black curve). Here we vary the lower bound $x_{b}$ of an $n=3$ ejecta velocity distribution. The blue curve corresponds to $x_{b}=5 \times 10^{-5}\left(v_{\mathrm{ej}} \sim 1\right.$ $\left.\mathrm{m} \mathrm{s}^{-1}\right)$, the red curve to $x_{b}=2 \times 10^{-5}\left(\sim 0.4 \mathrm{~m} \mathrm{~s}^{-1}\right)$ and the green curve to $x_{b}=2 \times 10^{-4}$ $\left(\sim 4 \mathrm{~m} \mathrm{~s}^{-1}\right)$. The effect of lowering $x_{b}$ is somewhat similar to increasing the viscosity (BT is weaker because of a shorter throw distance). In this case, $x_{b}=5 \times 10^{-5}$ appears to provide a better fit to the plateaus' outer edges. The largest $x_{b}$ demonstrates how the larger throw distances (and thus angular momentum disparities) lead to more pileup of material along with more radial drift. In the lower panel, the compositional profiles with smaller $x_{b}$ show the most variation because the bulk of material does not travel as far from its point of ejection.

Figure 14: Evolution over $2 \mathrm{t}_{G}$ of plateau features centered at $1.495 \mathrm{R}_{S}$ with $Y=10^{6}$, and $x_{b}=10^{-4}$ for different viscosity models (Sec. 2.2.7). The black curve corresponds to a purely kinematic viscosity model, whereas the red and blue curves correspond to combined models with different ring particle densities that include a viscosity due to wake formation. In all cases, $f_{\nu}=1$ for the kinematic viscosity. The condition for wake formation is marginally satisfied in the $\mathrm{C}$ ring plateaus. Compared to the purely kinematic viscosity model, the higher viscosity in the plateaus is very effective at suppressing the large amplitude growth of structure there despite such a high yield.

Figure A-1: Illustration that demonstrates the relationship between the "radiative transfer" angles $(\theta, \phi)$ and the cone and clock angles of emergent ejecta, $(\alpha, \beta)$. The angles are defined in a frame rotating with the ring material where $\alpha$ is measured from the orbital velocity vector $\mathbf{v}_{K}$, and $\beta$ from the ring normal. Reproduced from CD90.

Figure A-2: An example of actual model calculations of the ejecta distribution function $\mathscr{Y}$ at $R=1.8 \mathrm{R}_{S}$, which is plotted for two different optical depths as a function of the cone and clock angles $\alpha$ and $\beta$ (in radians) measured from the ring particle velocity vector. These models are averaged over ring orbit longitude. The prograde bias of the distribution is illustrated by the maximum occurring for $\alpha<1.5$. The parameterized fit we use in the 
BT code (Eq. [A-1]) matches the actual calculations within 10\%. Reproduced from CD90.

Figure C-1: Plot of the magnitude of the theoretical viscosity as a function of radius in the rings for a purely kinematic viscosity model (bold curve, Eq. [24]) which is $\tau$-dependent, and the viscosity model due to wakes (when greater, lighter curve, Eq. [C-1]) which depends on $\sigma$. The UVIS Cassini $\alpha$-Arae occultation data defines $\tau$, and a variable opacity is used. The density of ring particles for the wake viscosity is assumed to be $0.5 \mathrm{~g} \mathrm{~cm}^{-3}$, while $\bar{r}=64.6$ $\mathrm{cm}$ for the kinematic viscosity. The condition for wake formation can be marginally satisfied in the $\mathrm{C}$ ring plateaus, especially if the mean density of particles is higher. Outside of the plateaus, the viscosity is purely kinematic. Some evidence exists for wakes in the inner B ring, which would mean much larger viscosities than given by a purely kinematic model. This could pose a problem for maintaining a sharp edge by BT alone, but the situation is complicated (see Sec. 2.2.7). 
Parameter Definition

\begin{tabular}{|c|c|}
\hline $\mathscr{A}$ & Probability of meteoroid impact for given $\tau$ \\
\hline$B$ & Elevation angle \\
\hline$D$ & $\begin{array}{l}\text { Edge width parameter for inner } \mathrm{B} \text { ring edge } \\
\text { models }\end{array}$ \\
\hline$f$ & Ejecta speed distribution \\
\hline$f_{\mathrm{e}}$ & Total mass fraction of extrinsic pollutant \\
\hline$f_{\text {ext }}$ & Mass fraction of extrinsic, absorbing material \\
\hline$F_{\mathrm{G}}$ & Gravitational focusing factor \\
\hline$f_{\nu}$ & Kinematic viscosity scaling factor \\
\hline$h, h_{c}$ & Specific angular momentum per unit mass \\
\hline$\Gamma_{\mathrm{m}, \mathrm{h}}, \Lambda_{\mathrm{m}, \mathrm{h}}$ & $\begin{array}{l}\text { Direct gains and losses in mass and angular } \\
\text { momentum }\end{array}$ \\
\hline$\Gamma_{\mathrm{m}, \mathrm{e}}, \Gamma_{\mathrm{m}, \mathrm{i}}$ & $\begin{array}{l}\text { Fractional mass gain integrals for extrinsic and } \\
\text { intrinsic materials }\end{array}$ \\
\hline$\eta$ & $\begin{array}{l}\text { Retention efficiency of absorbing properties fol- } \\
\text { lowing impact }\end{array}$ \\
\hline$\kappa$ & Opacity $=\tau / \sigma$ \\
\hline$\nu$ & Ring viscosity \\
\hline$N$ & Number of Lagrangian (annuli) bins \\
\hline$n$ & Powerlaw exponent for $f$ \\
\hline $\mathscr{P}$ & Ejecta absorption probability \\
\hline$r$ & Ring particle radius \\
\hline$r_{1}, r_{2}$ & $\begin{array}{l}\text { Upper and lower bound of particle size } \\
\text { distribution }\end{array}$ \\
\hline $\bar{r}$ & $\begin{array}{l}\text { Mean particle radius above which } 1 / 3 \text { of the op- } \\
\text { tical depth is accounted for }\end{array}$ \\
\hline$R$ & Radial distance from the central planet \\
\hline$\rho$ & Ring particle density \\
\hline$\sigma$ & Surface mass density \\
\hline$\dot{\sigma}_{\mathrm{ej}}$ & Ejecta mass flux \\
\hline$\dot{\sigma}_{\text {im }}$ & Two sided mass flux impacting within the rings \\
\hline$\dot{\sigma}_{\infty}$ & Unfocussed, one-sided, interplanetary mass flux \\
\hline$\tau$ & Normal optical depth \\
\hline$t_{\mathrm{G}}$ & Gross erosion time \\
\hline$v_{\mathrm{R}}$ & Total radial drift velocity \\
\hline$x$ & Ratio of ejecta velocity to orbital velocity \\
\hline$x_{b}$, & Lower and upper bounds of ejecta distribution \\
\hline$Y$ & Impact ejecta yield \\
\hline $\mathscr{Y}$ & Distribution of ejecta with angle and optical depth \\
\hline
\end{tabular}



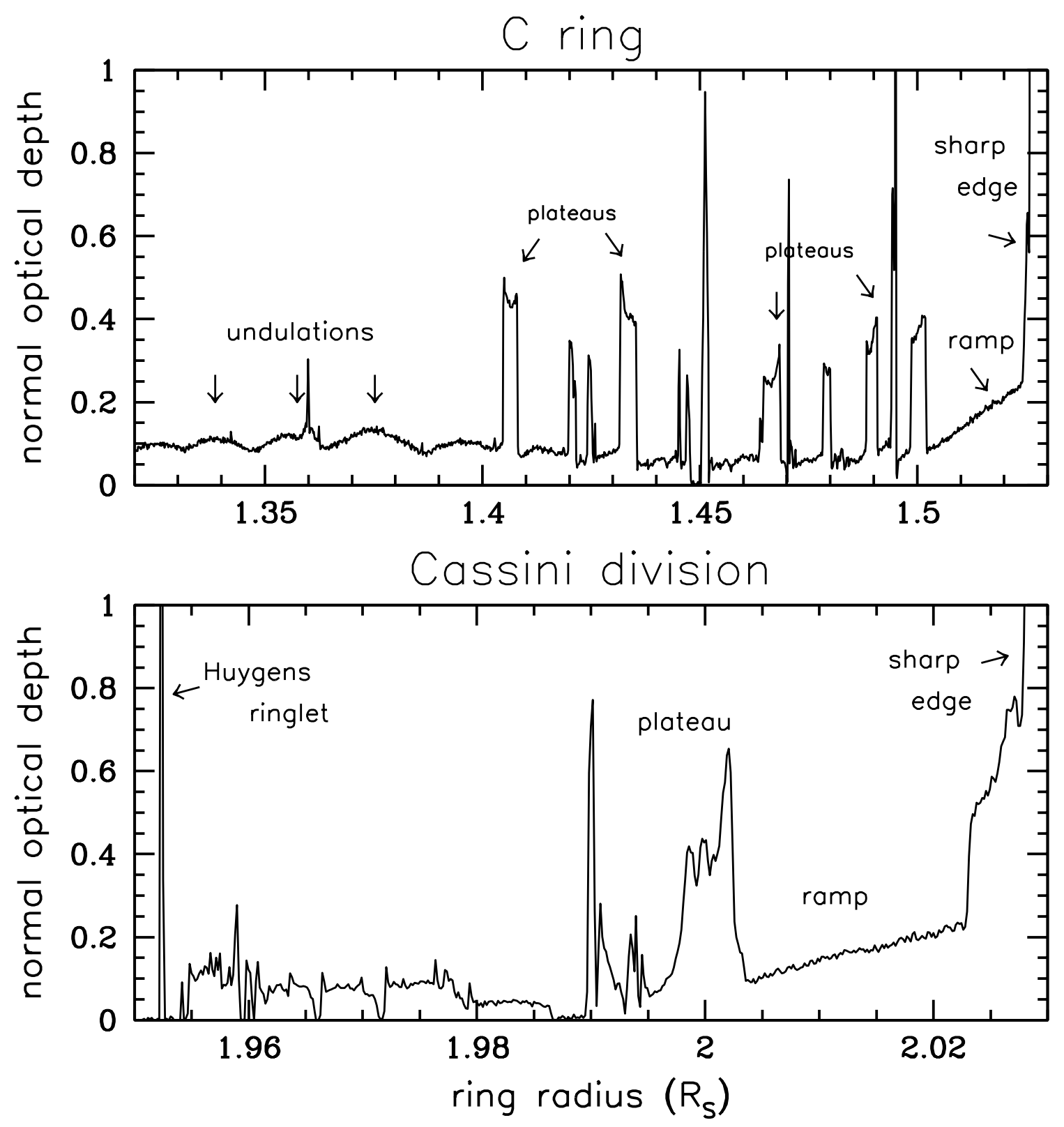

Figure 1: Estrada et al. 


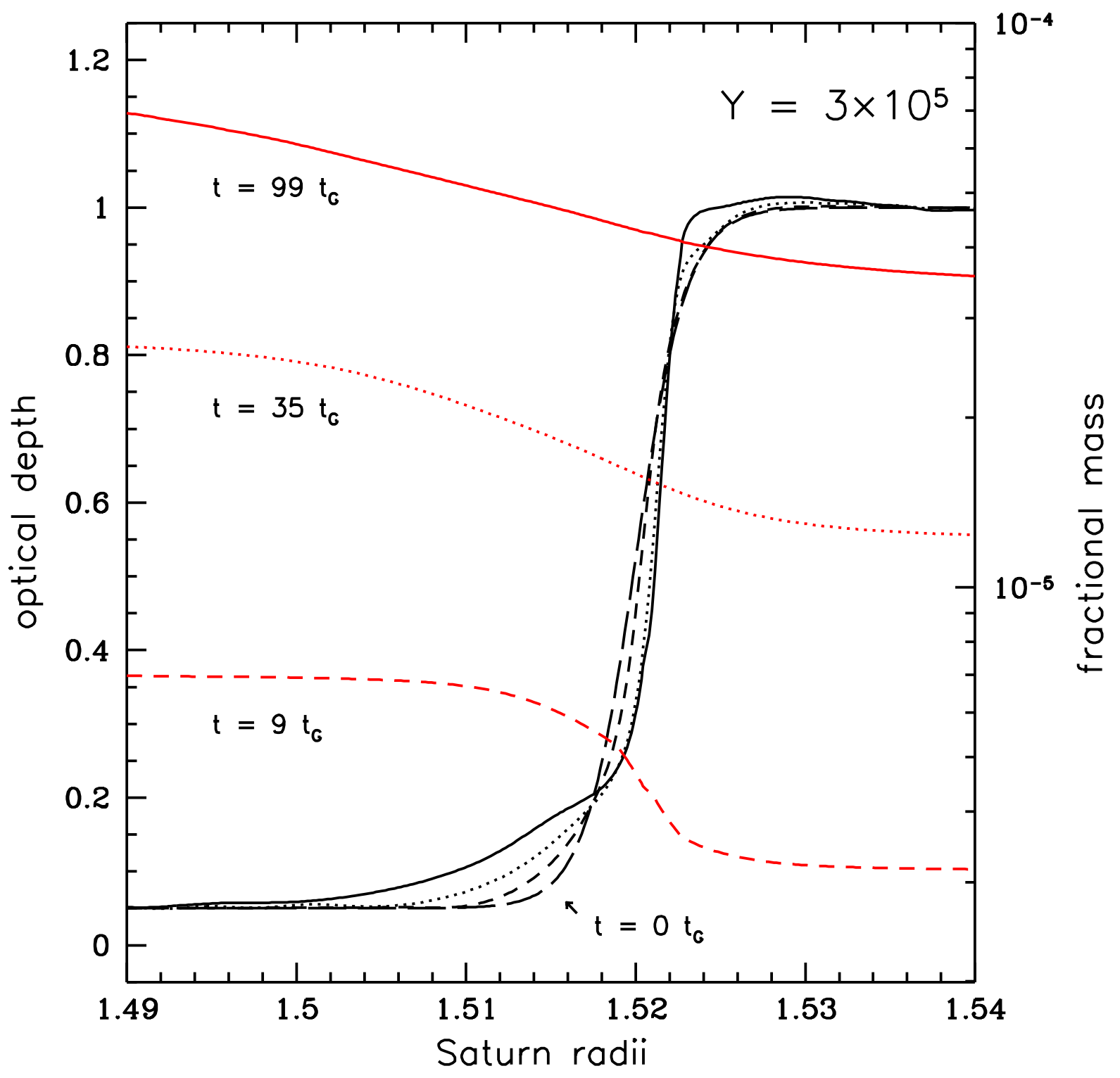

Figure 2: Estrada et al. 


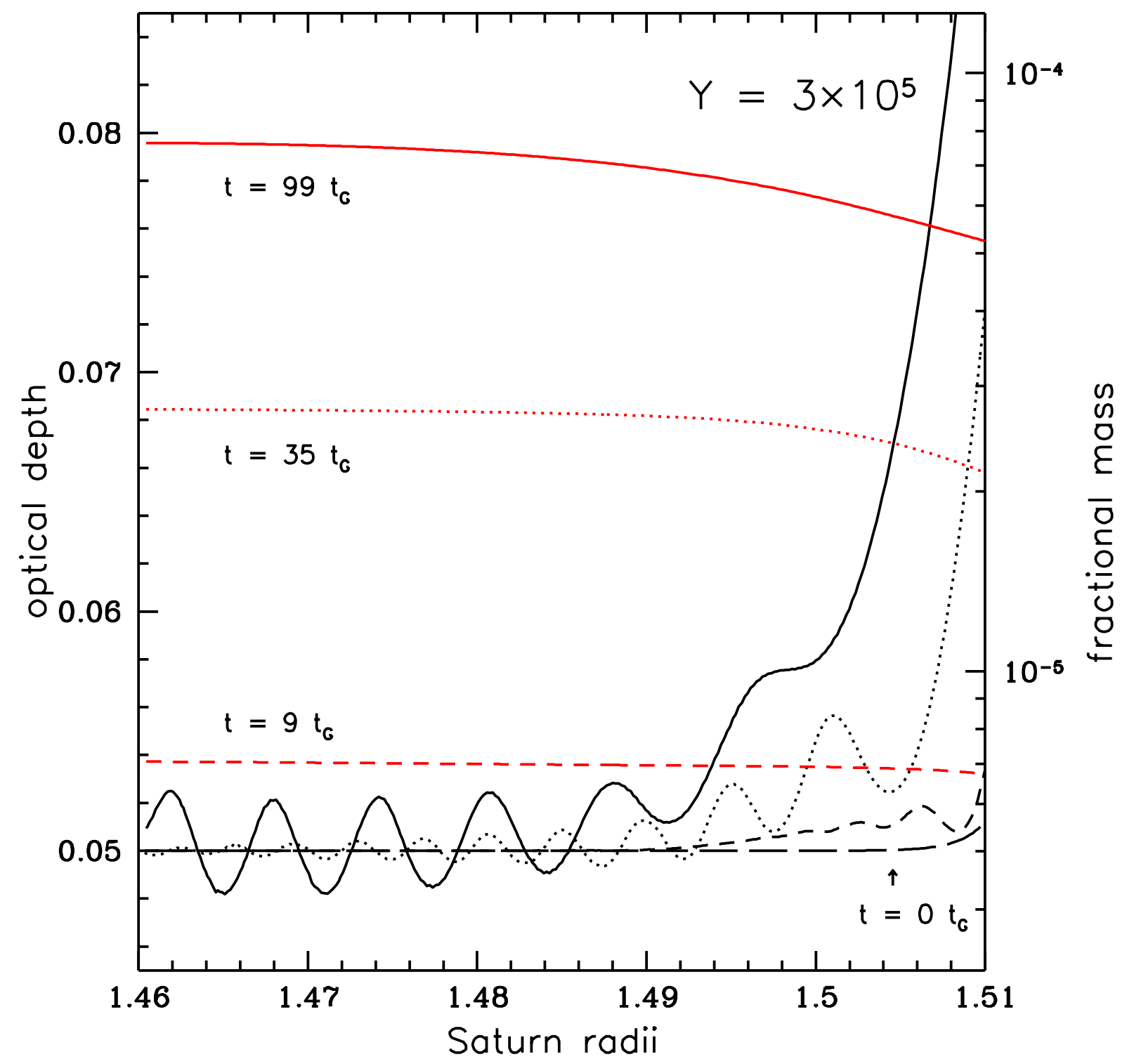

Figure 3: Estrada et al. 


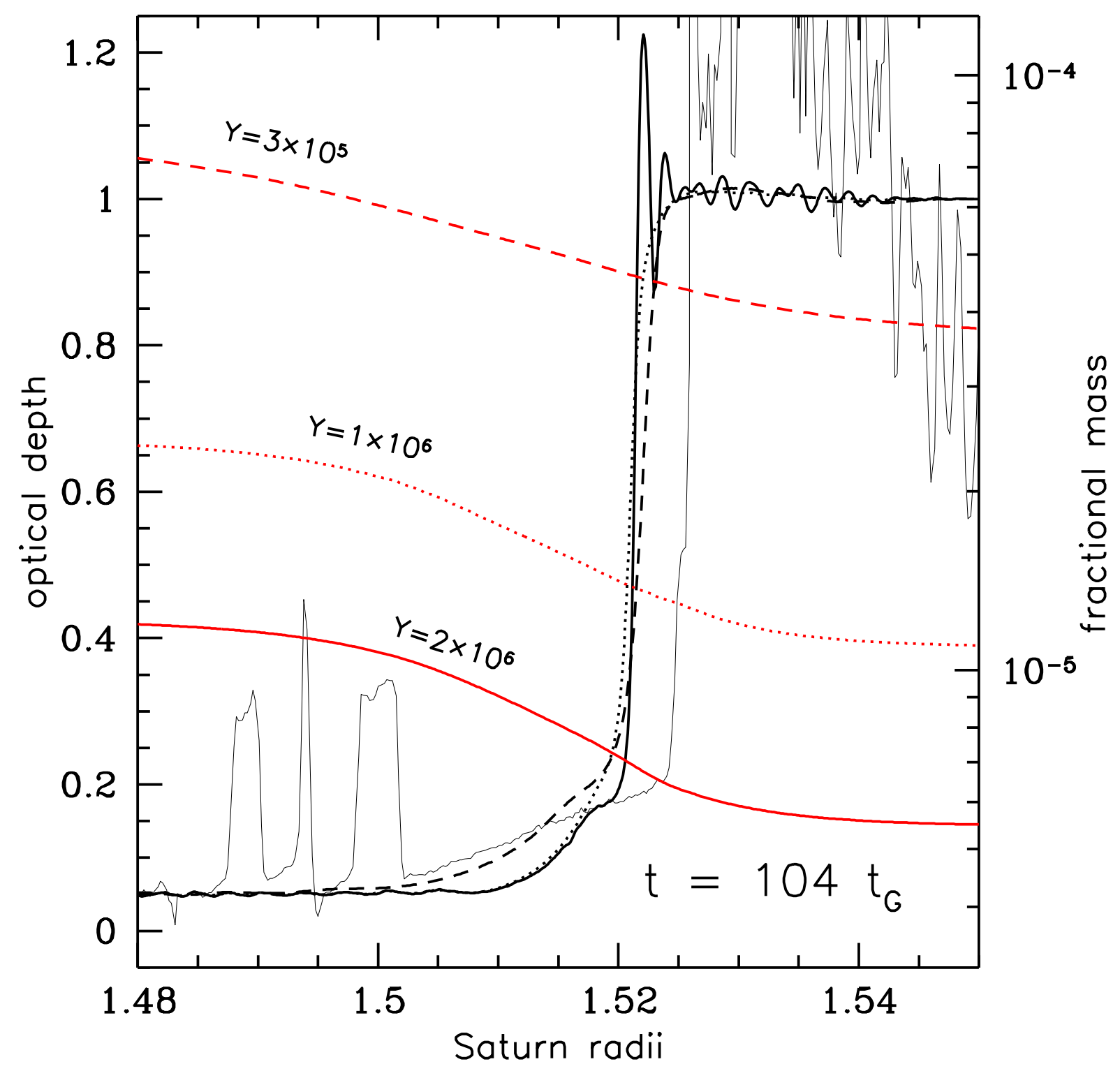

Figure 4: Estrada et al. 


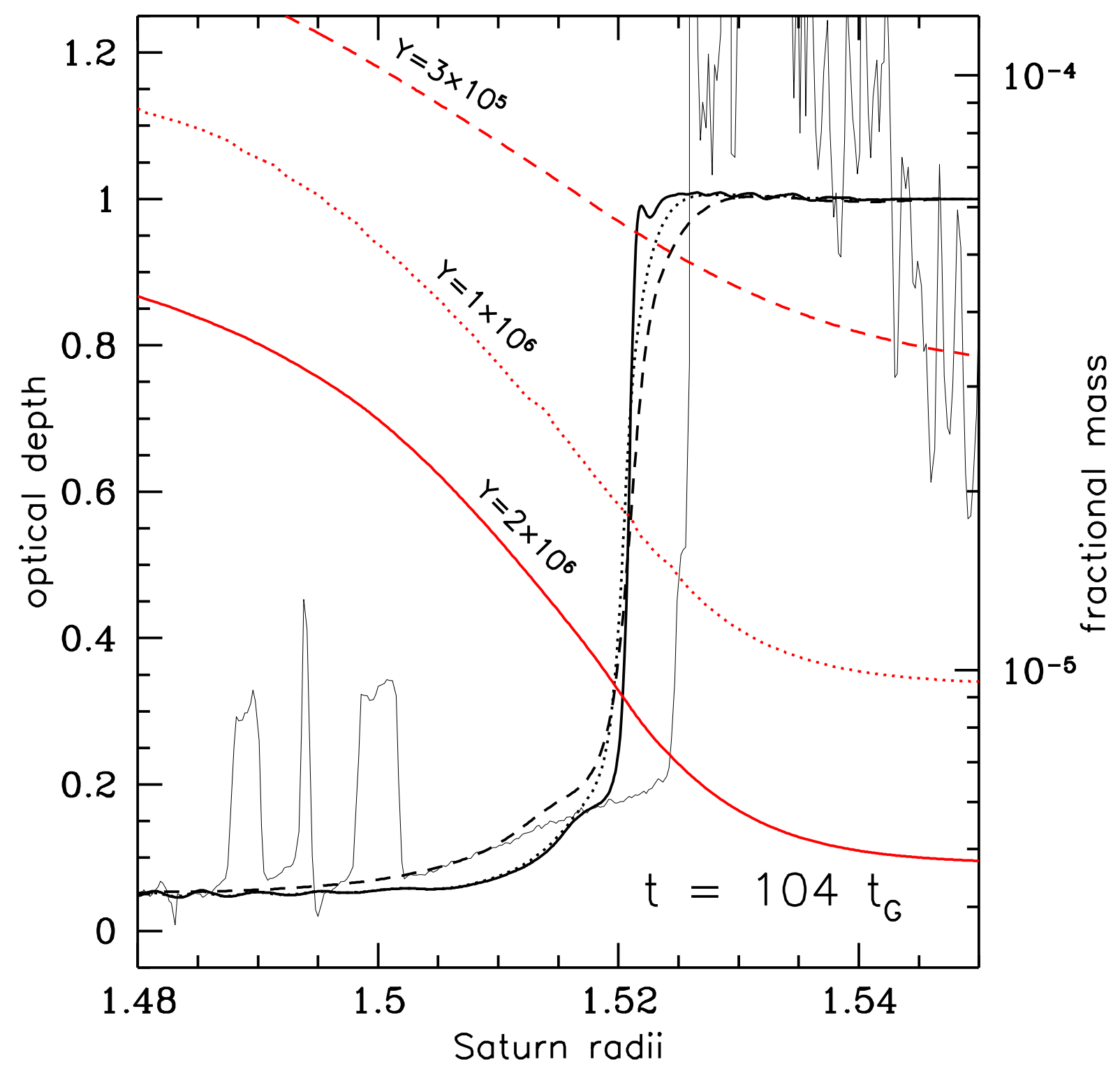

Figure 5: Estrada et al. 


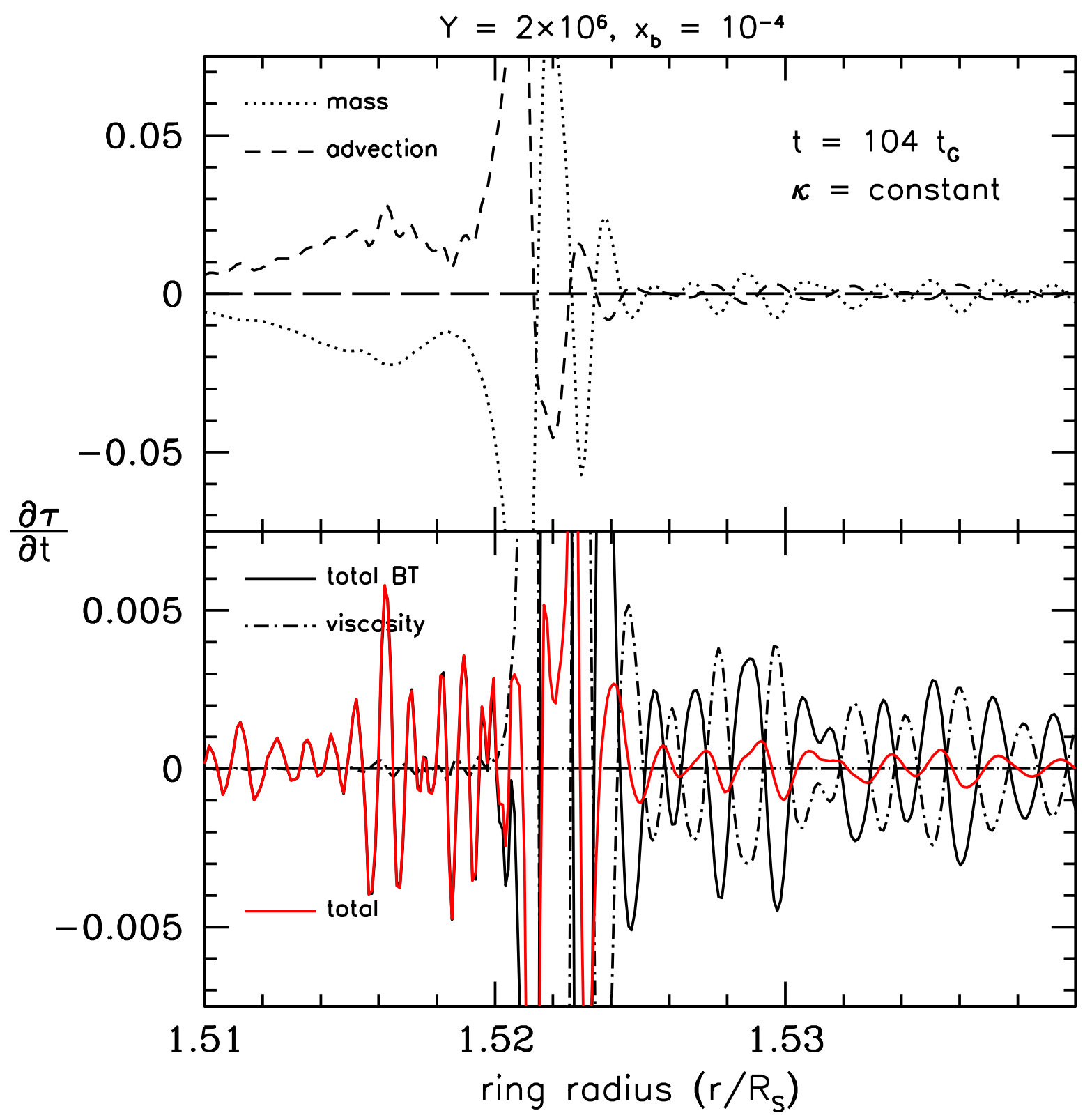

Figure 6: Estrada et al. 


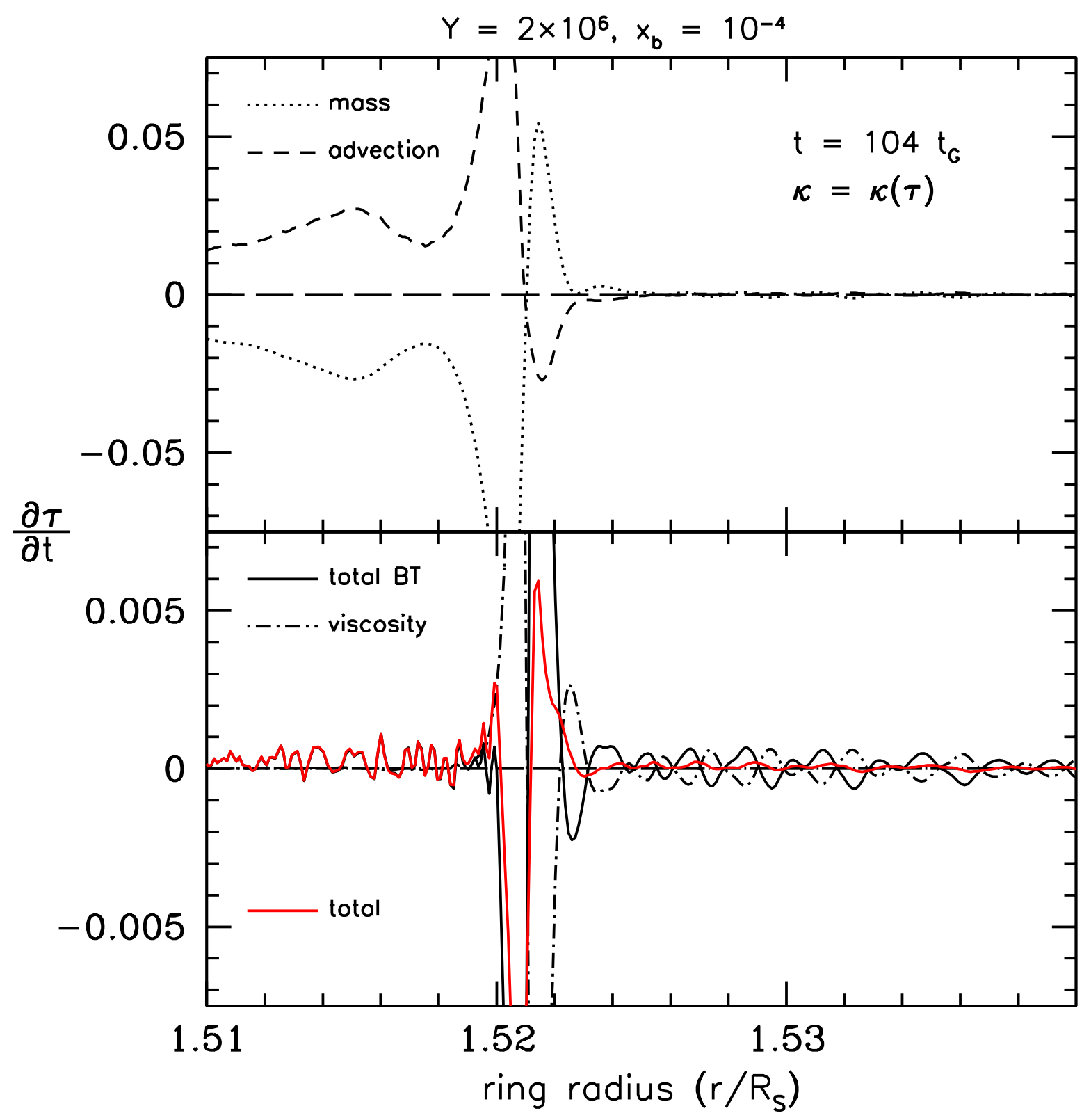

Figure 7: Estrada et al. 


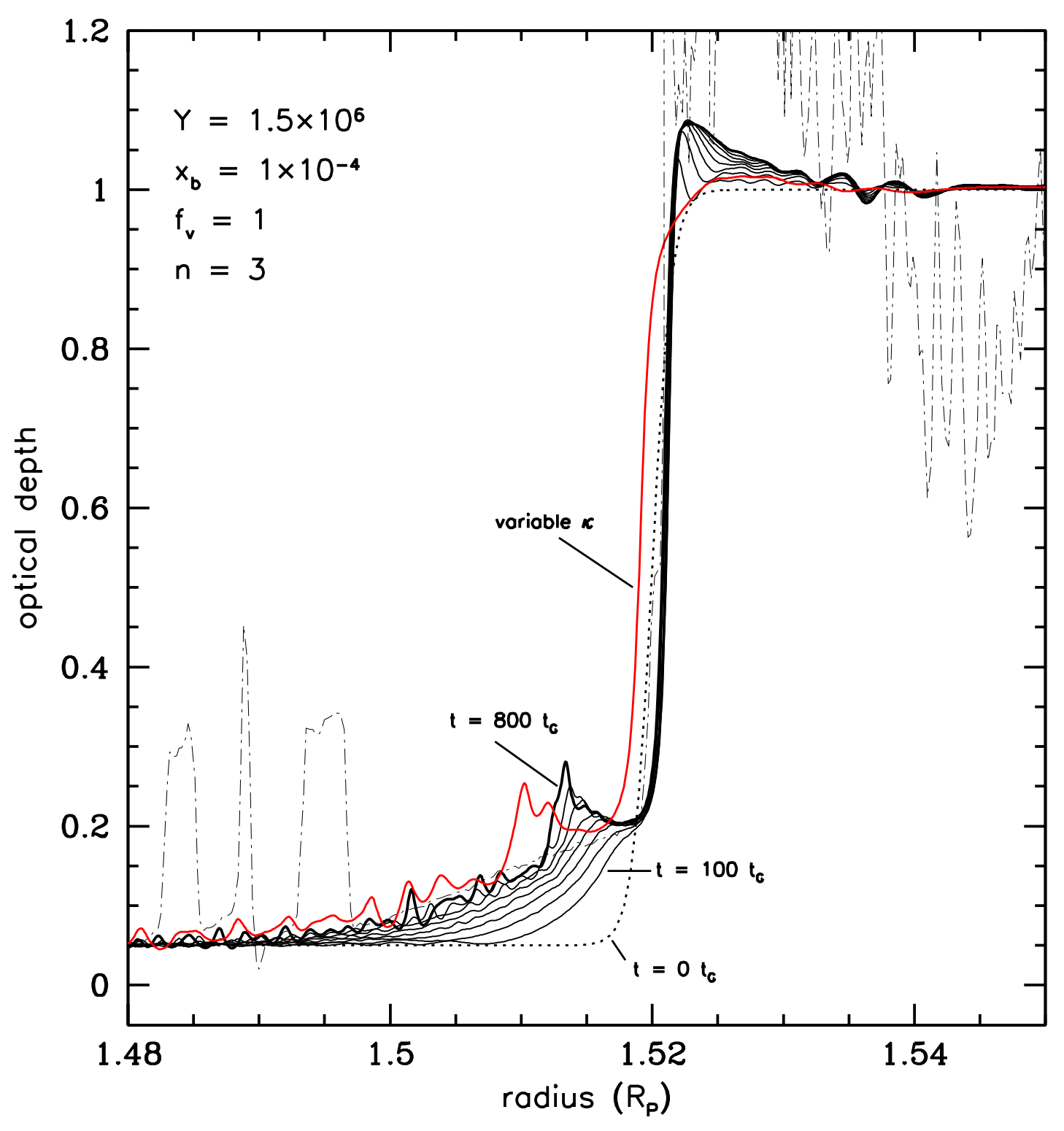

Figure 8: Estrada et al. 


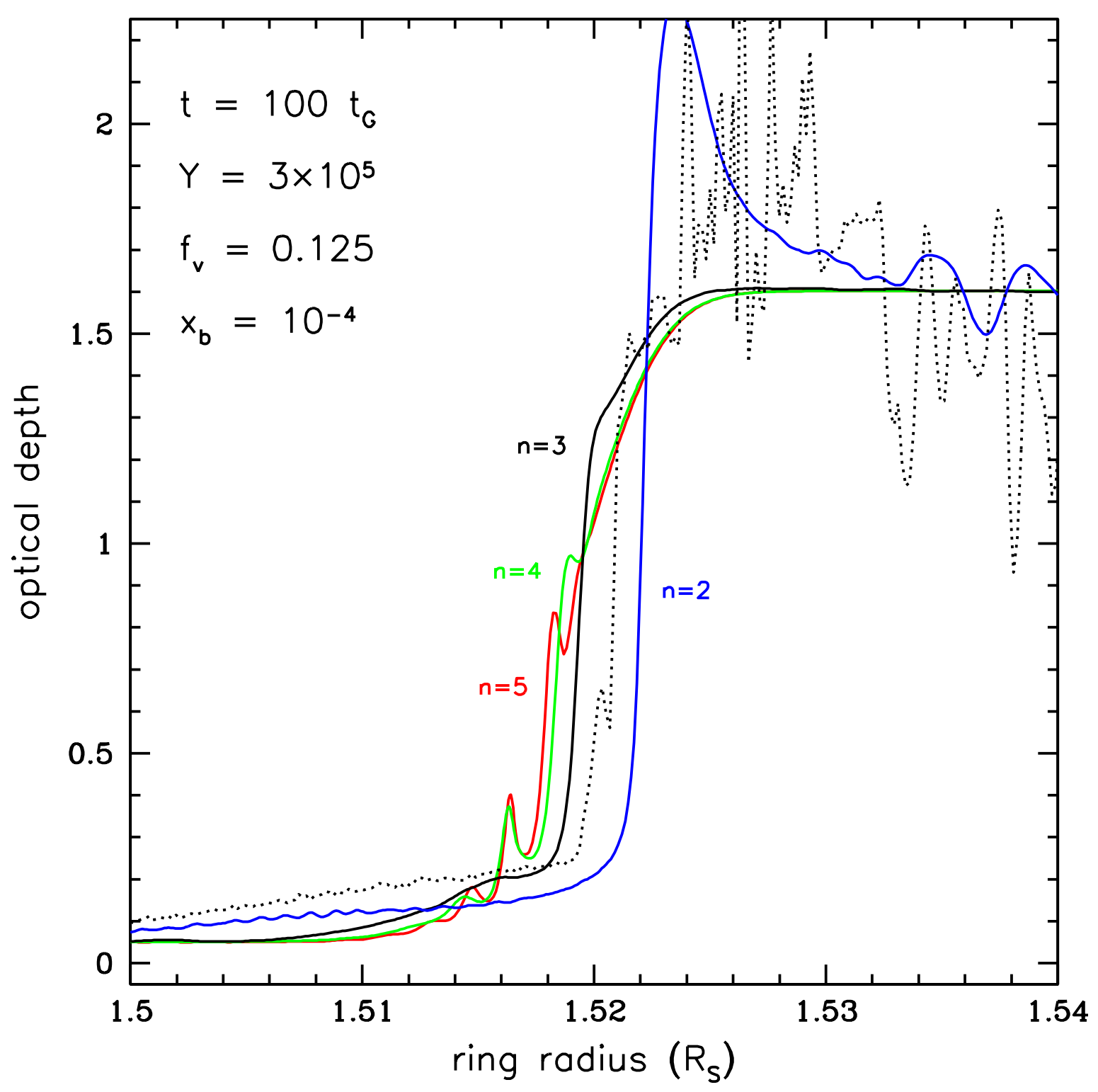

Figure 9: Estrada et al. 


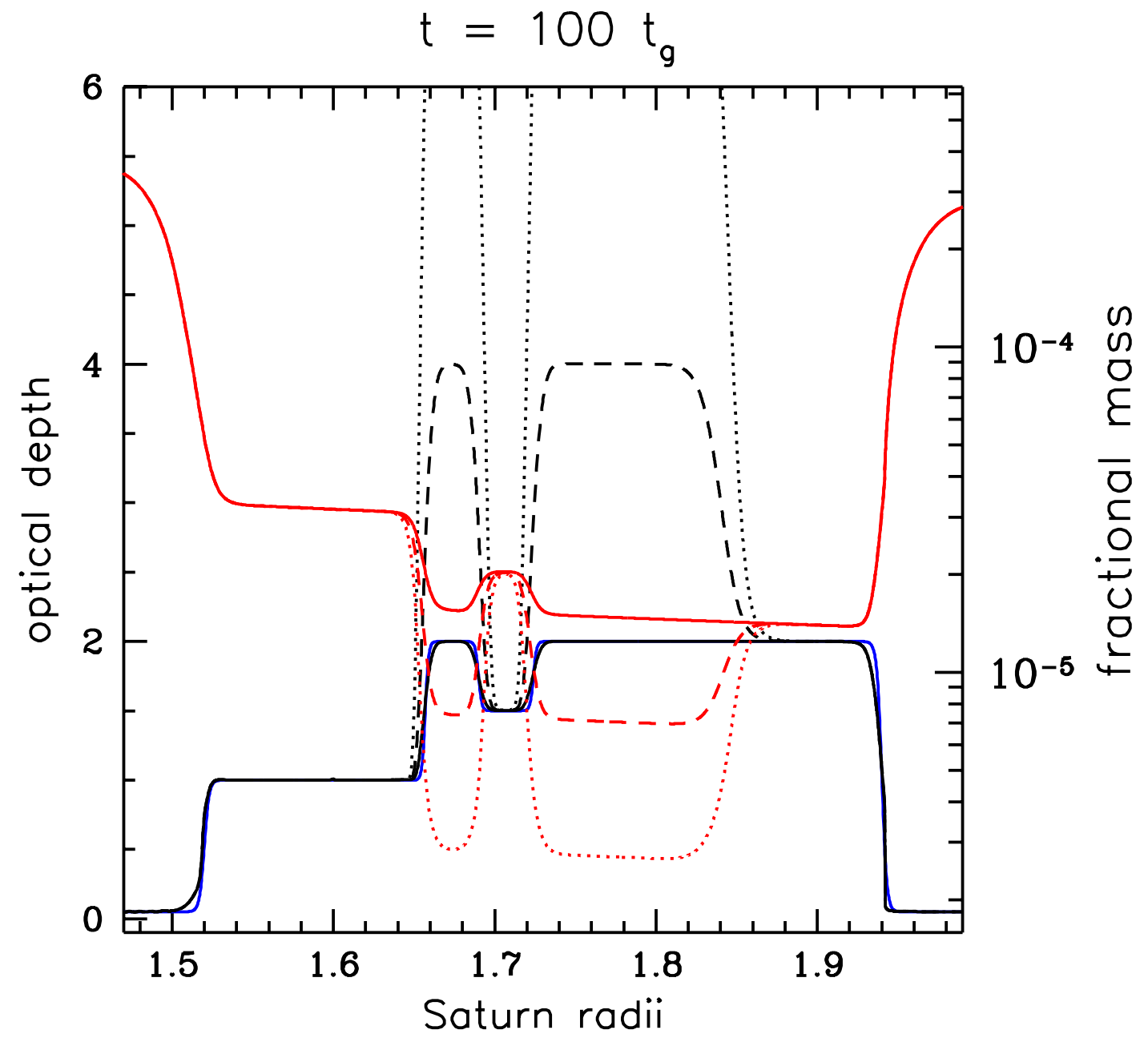

Figure 10: Estrada et al. 

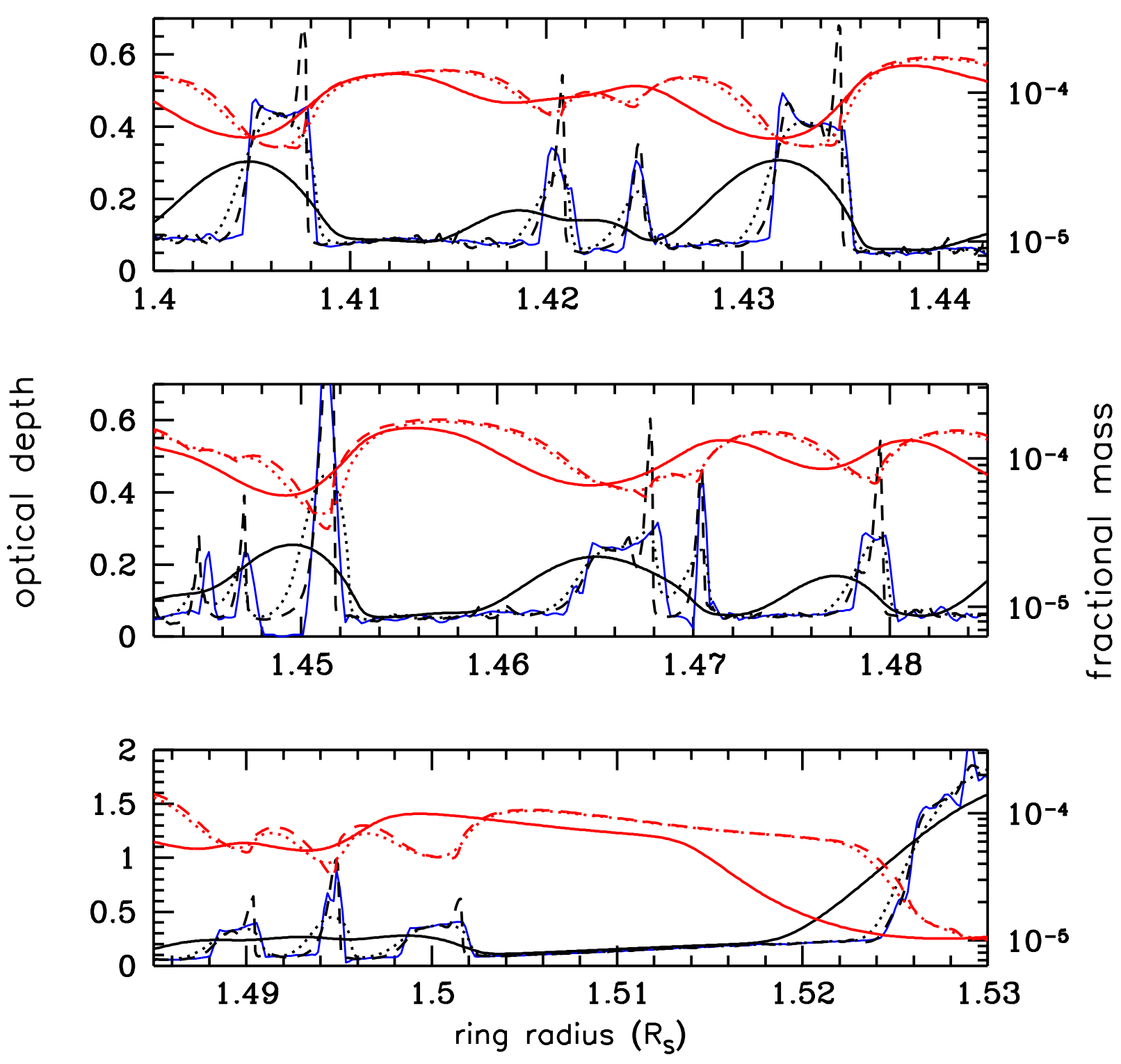

Figure 11: Estrada et al. 


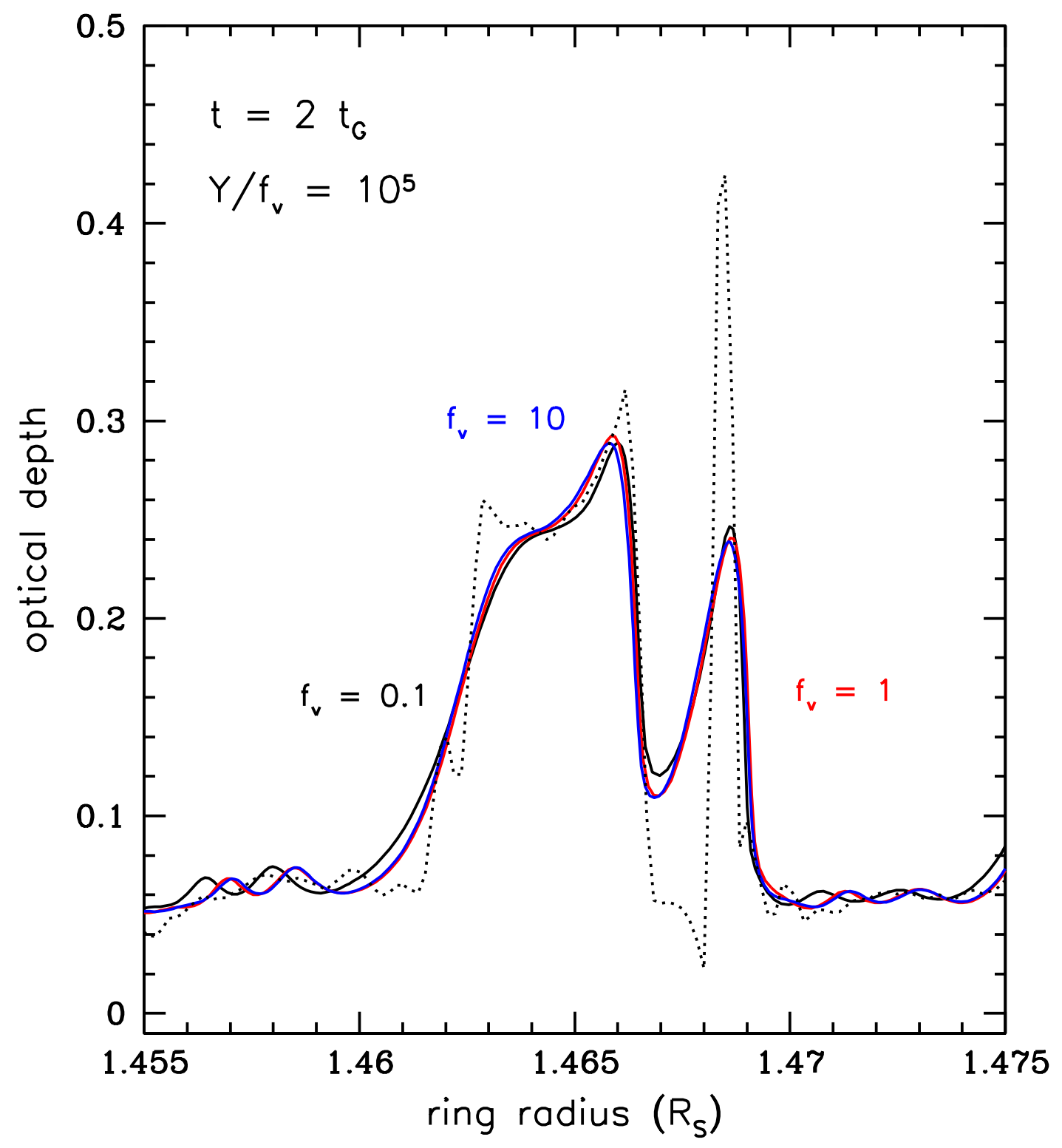

Figure 12: Estrada et al. 

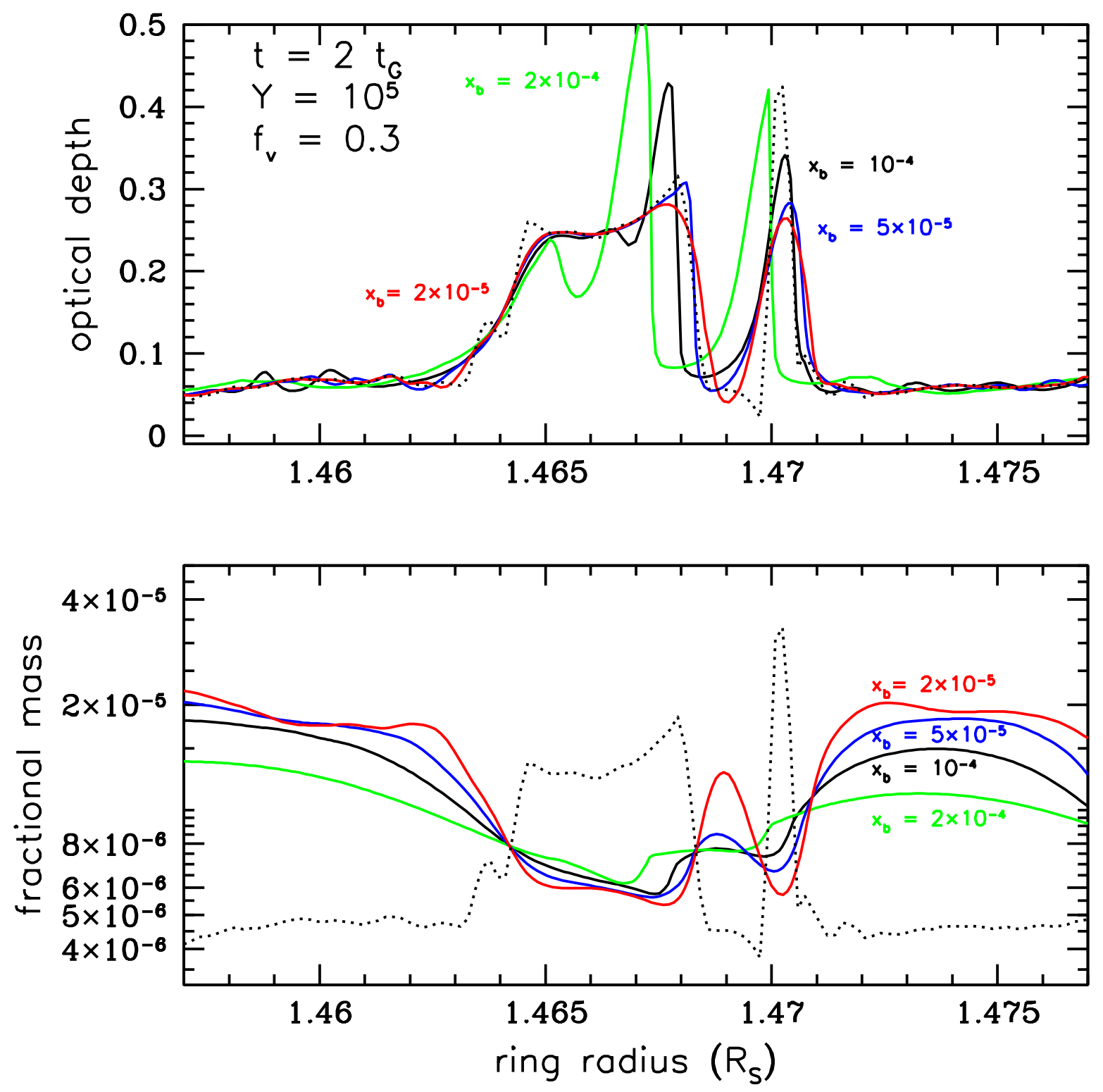

Figure 13: Estrada et al. 


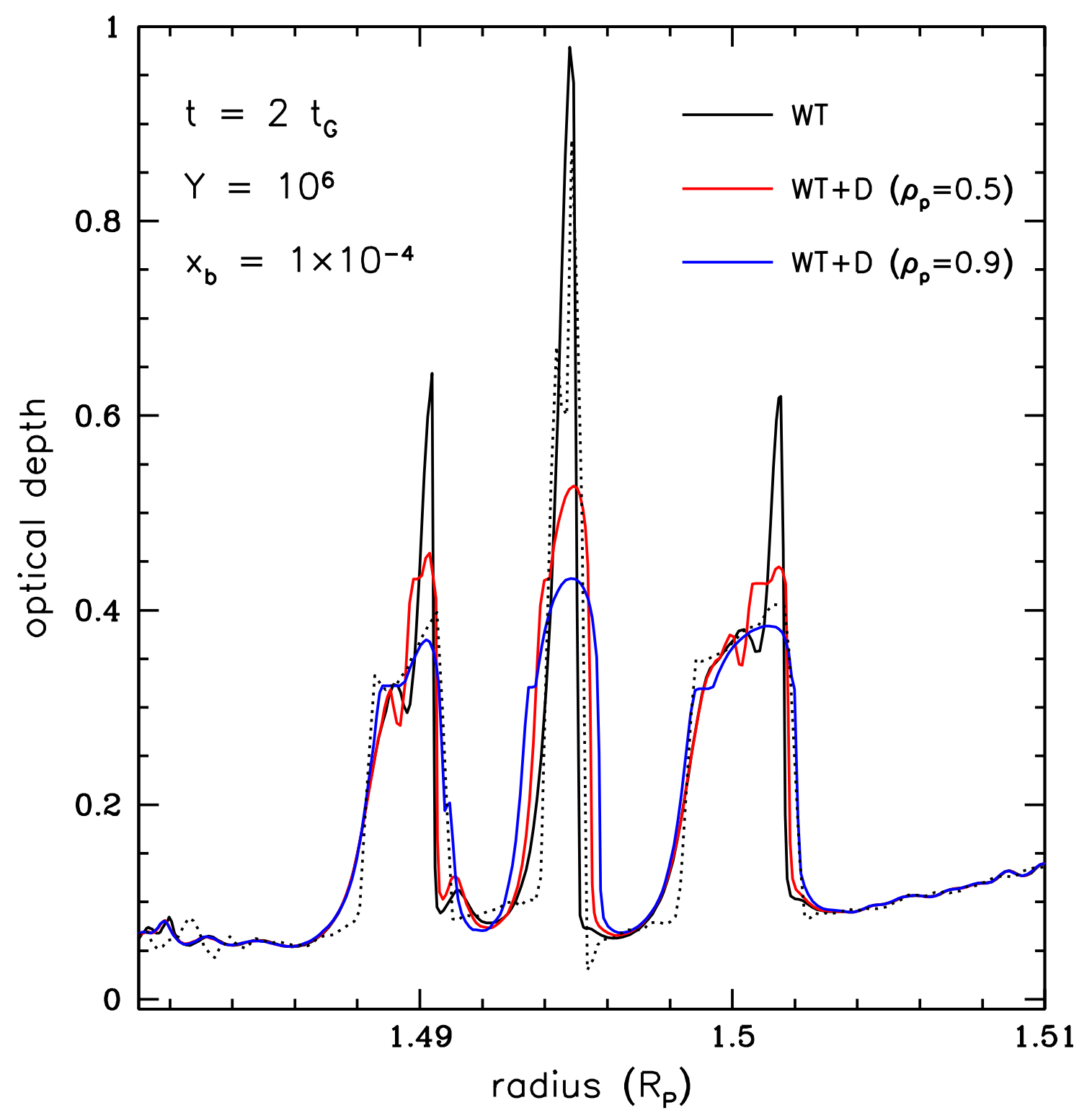

Figure 14: Estrada et al. 


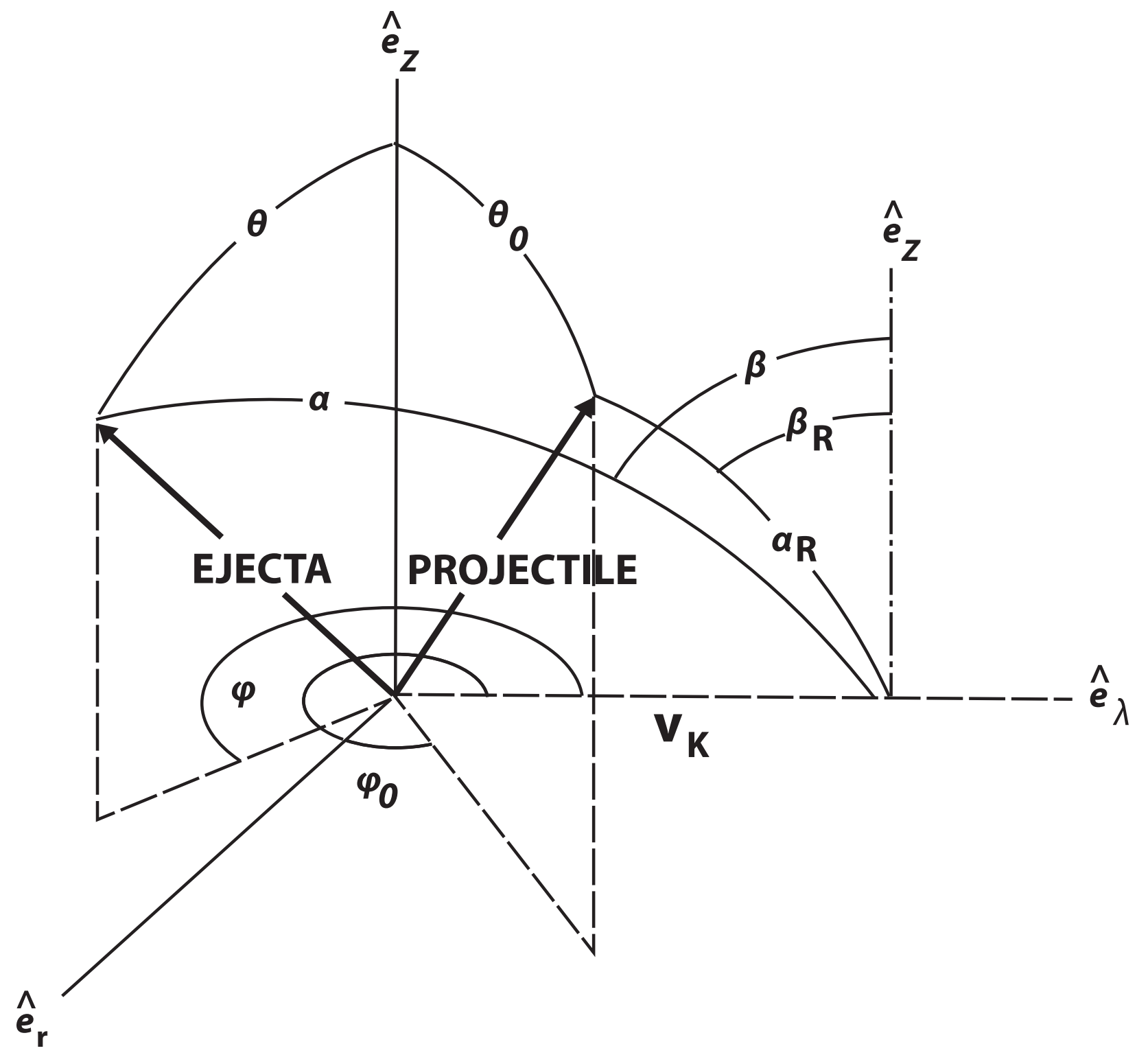

Figure A-1: Estrada et al. 

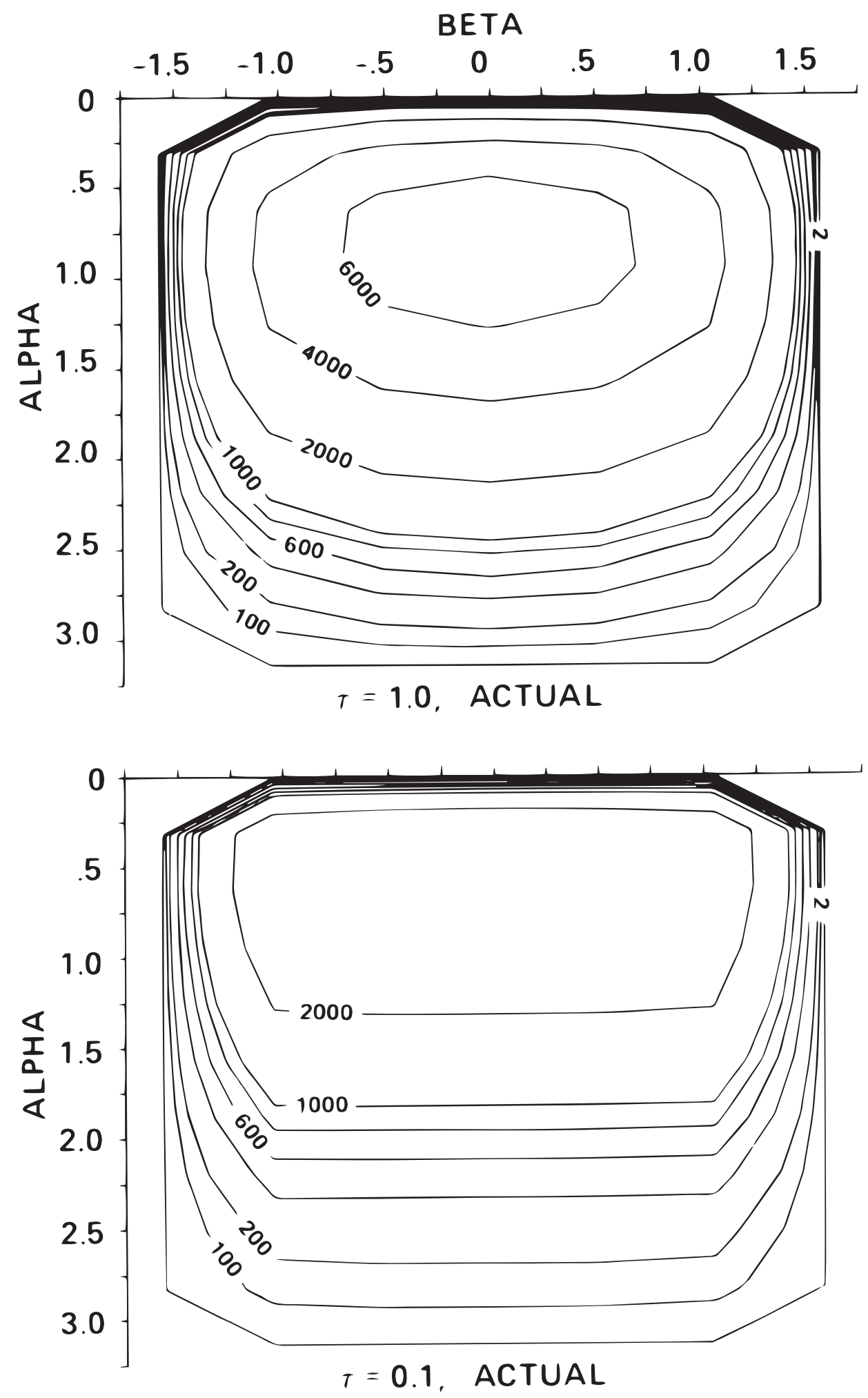

Figure A-2: Estrada et al. 


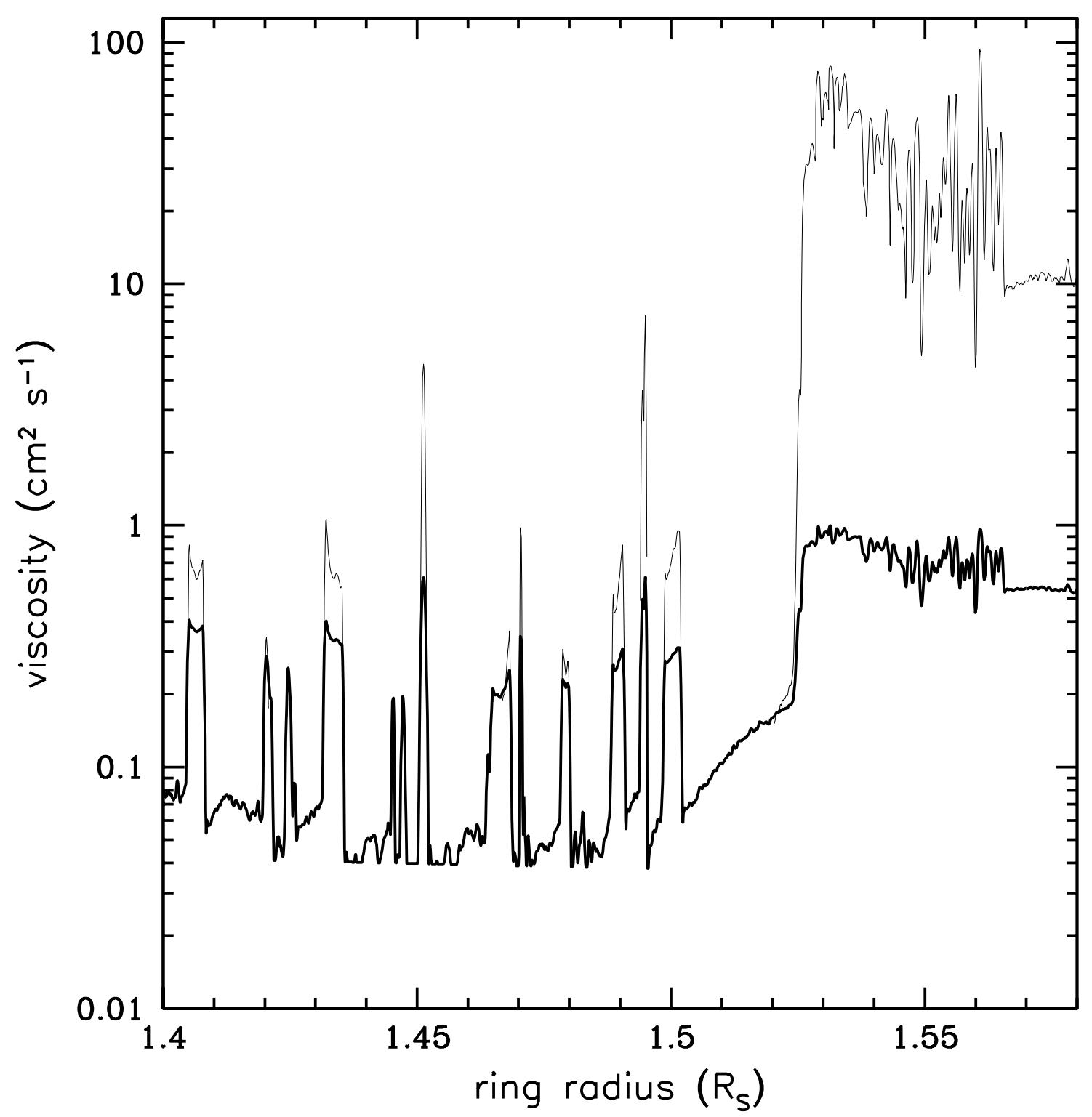

Figure C-1: Estrada et al. 